\title{
Toward Highly Potent Cancer Agents by Modulating the C-2 Group of the Arylthioindole Class of Tubulin Polymerization Inhibitors
}

\author{
Giuseppe La Regina ${ }^{\dagger}$, Ruoli Bai ${ }^{\ddagger}$, Whilelmina Maria Rensen ${ }^{\S}$, Erica Di Cesare $§$, Antonio \\ Coluccia $^{\dagger}$, Francesco Piscitelli ${ }^{\dagger}$, Valeria Famiglini ${ }^{\dagger}$, Alessia Reggio ${ }^{\dagger}$, Marianna Nalli ${ }^{\dagger}$, \\ Sveva Pelliccia ${ }^{\bullet}$, Eleonora Da Pozzoll, Barbara Costall, Ilaria Granata ${ }^{\perp}$, Amalia Porta $^{\perp}$, \\ Bruno Maresca ${ }^{\perp}$, Alessandra Soriani ${ }^{\times}$, Maria Luisa lannitto ${ }^{\times}$, Angela Santoni ${ }^{\S}, \times$, Junjie Li $^{\#}$, \\ Marlein Miranda Cona\#, Feng Chen" ${ }^{\#}$, Yicheng Ni", Andrea Brancale ${ }^{\infty}$, Giulio Dondio ${ }^{\circ}$, \\ Stefania Vultaggio ${ }^{\star}$, Mario Varasi ${ }^{\star}$, Ciro Mercurio ${ }^{\triangle}$, Claudia Martinill, Ernest Hamel ${ }^{\ddagger}$, \\ Patrizia Lavia§, Ettore Novellino ${ }^{*}$, and Romano Silvestri ${ }^{*}, \dagger$ \\ †Dipartimento di Chimica e Tecnologie del Farmaco, Istituto Pasteur-Fondazione Cenci \\ Bolognetti, Sapienza Università di Roma, Piazzale Aldo Moro 5, I-00185 Roma, Italy \\ ¥Screening Technologies Branch, Developmental Therapeutics Program, Division of Cancer \\ Treatment and Diagnosis, Frederick National Laboratory for Cancer Research, National Cancer \\ Institute, National Institutes of Health, Frederick, Maryland 21702, United States \\ $\S$ Institute of Molecular Biology and Pathology (IBPM), CNR National Research Council of Italy, c/o \\ Sapienza Università di Roma, Via degli Apuli 4, I-00185 Roma, Italy \\ "Department of Psychiatry, Neurobiology, Pharmacology, and Biotechnology, University of Pisa, \\ Via Bonanno Pisano 6, I-56126 Pisa, Italy \\ ${ }^{\perp}$ Dipartimento di Scienze Farmaceutiche, Sezione Biomedica, Università di Salerno, Via Ponte \\ don Melillo, I-84084 Fisciano, Salerno, Italy \\ \#Theragnostic Laboratory, Department of Imaging and Pathology, Faculty of Medicine, \\ Biomedical Sciences Group, KU Leuven, Herestraat 49, B-3000 Leuven, Belgium
}

${ }^{\infty}$ Welsh School of Pharmacy, Cardiff University, King Edward VII Avenue, Cardiff, CF10 3NB, U.K

-Dipartimento di Chimica Farmaceutica e Tossicologica, Università di Napoli Federico II, Via Domenico Montesano 49, I-80131, Napoli, Italy

×Dipartimento di Medicina Sperimentale e Patologia, Sapienza Università di Roma, Viale Regina Elena 324, I-00161 Roma, Italy

'NiKem Research Srl, Via Zambeletti 25, I-20021 Baranzate, Milano, Italy

-European Institute of Oncology, Via Adamello 16, I-20139 Milano, Italy

${ }^{\triangle}$ Genextra Group, DAC SRL, Via Adamello 16, I-20139 Milano, Italy

\author{
(C) 2012 American Chemical Society \\ *Corresponding Author: Phone: +39 064991 3800. Fax: +39 064991 3133. romano.silvestri@uniroma1.it. \\ The authors declare no competing financial interest. \\ ASSOCIATED CONTENT \\ Supporting Information \\ Molecular modeling results, binding modes, inhibition data, cell death data, and elemental analysis results. This material is available \\ free of charge via the Internet at http://pubs.acs.org.
}




\section{Abstract}

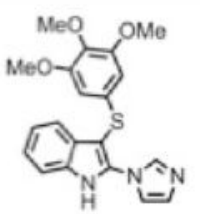

18

Tubulin $\left(I C_{g y} \mu M\right)$ MCF-7 (IC HLM Stability (\% rem.)

Solubility $(\mathrm{pH} 7.4, \mu \mathrm{M})$
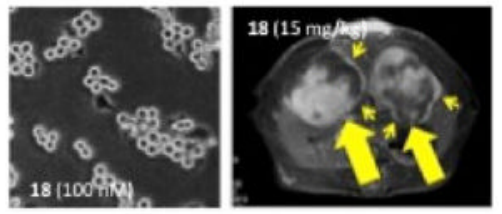

18

0.95

1.0

49

65

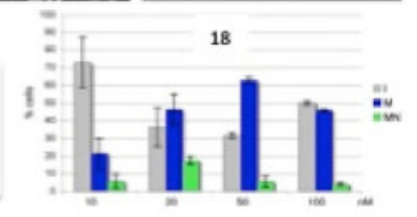

New arylthioindole derivatives having different cyclic substituents at position 2 of the indole were synthesized as anticancer agents. Several compounds inhibited tubulin polymerization at submicromolar concentration and inhibited cell growth at low nanomolar concentrations. Compounds 18 and $\mathbf{5 7}$ were superior to the previously synthesized $\mathbf{5}$. Compound $\mathbf{1 8}$ was exceptionally potent as an inhibitor of cell growth: it showed $\mathrm{IC}_{50}=1.0 \mathrm{nM}$ in MCF-7 cells, and it was uniformly active in the whole panel of cancer cells and superior to colchicine and combretastatin A-4. Compounds $\mathbf{1 8}, \mathbf{2 0}, \mathbf{5 5}$, and $\mathbf{5 7}$ were notably more potent than vinorelbine, vinblastine, and paclitaxel in the NCI/ADR-RES and Messa/Dx5 cell lines, which overexpress Pglycoprotein. Compounds $\mathbf{1 8}$ and $\mathbf{5 7}$ showed initial vascular disrupting effects in a tumor model of liver rhabdomyosarcomas at $15 \mathrm{mg} / \mathrm{kg}$ intravenous dosage. Derivative $\mathbf{1 8}$ showed water solubility and higher metabolic stability than $\mathbf{5}$ in human liver microsomes.

\section{INTRODUCTION}

Microtubules (MTs) are involved in many essential cellular functions, e.g., the maintenance of cell shape, cell motility, intracellular transport, and cell division. Cellular MTs undergo continuous polymerization and depolymerization transitions. Interference with this dynamic equilibrium, by either inhibiting tubulin polymerization or blocking MT disassembly, prevents proper MT function and ultimately leads to cell death. Because of their crucial role in the formation of the mitotic spindle during cell division, MTs are a highly attractive target for the development of new effective anticancer agents. ${ }^{1-5}$

Natural products such as colchicine (1),${ }^{6,7}$ combretastatin A-4 (CSA4, 2) $)^{8}$ (Chart 1), and the Catharanthus alkaloids vincristine and vinblastine (VBL) inhibit MT assembly by preventing tubulin polymerization. On the other hand, taxoids and epothilones target a lumenal site on the $\beta$-subunit. ${ }^{9,10}$ These drugs enter the lumen through a binding site ${ }^{11}$ located at a pore on the MT surface formed by different tubulin heterodimers. At high concentrations, paclitaxel (PTX) stimulates MT polymerization and stabilizes microtubules, whereas at lower concentrations it inhibits MT dynamics with little effect on the proportion of tubulin in polymer. ${ }^{12}$ Despite substantial interest in the development of new MT-targeting drugs, ${ }^{13}$ several problems related to drug resistance and secondary toxicity remain unresolved. Therefore, new and improved tubulin inhibitors are needed. ${ }^{14}$

Arylthioindoles (ATIs) are a class of potent inhibitors of tubulin polymerization and cancer cell growth developed by our research group. ${ }^{15-19}$ The ATIs inhibit tubulin polymerization by binding to the colchicine site, inhibiting the binding of $\left[{ }^{3} \mathrm{H}\right]$ colchicine to tubulin. ${ }^{15}$

In our previous work, ${ }^{19}$ we obtained new ATIs by replacing the 2-alkoxycarbonyl group with a bioisosteric five-membered heterocycle nucleus. The new ATIs (i.e., 3-6) inhibited 
tubulin polymerization, inhibited the growth of a panel of human transformed cell lines, and showed higher metabolic stability than the reference ester (see example in Chart 1).

These new ATIs showed significant therapeutic potential, as they induced mitotic arrest and apoptosis similarly to $\mathbf{2}$ and VBL and triggered caspase-3 expression in a significant fraction of cells in both p53-positive and p53-negative cell lines. ${ }^{20}$ Importantly, some ATIs were more effective than VRB, VBL, and PTX as growth inhibitors of the P-glycoproteinoverexpressing cell line NCI/ADR-RES. These compounds had a pharmacokinetic profile in the mouse characterized by low systemic clearance and excellent oral bioavailability. On the basis of these observations, the current study was designed to explore further chemical modifications of the five-membered heterocycle nucleus at position 2 of the indole. We describe the synthesis and biological evaluation of new ATI derivatives 7-78 with different biososteric rings at position 2, with a sulfur bridge between the ring systems or with other bioisosteric groups at position 3 of the indole (Table 1).

\section{CHEMISTRY}

ATI derivatives $17, \mathbf{1 8}, \mathbf{2 0}, \mathbf{2 7}, \mathbf{3 3}, \mathbf{3 4}, \mathbf{3 8}, \mathbf{3 9}, \mathbf{4 1}, \mathbf{4 3}, \mathbf{4 5}-\mathbf{4 7}, \mathbf{5 3}, \mathbf{5 5}, \mathbf{5 7}, \mathbf{7 3}, \mathbf{7 5}$, and 77 were synthesized by microwave reaction of an appropriate indole 79-87 with bis(3,4,5-trimethoxyphenyl)disulfide in the presence of sodium hydride in a closed vessel at $110{ }^{\circ} \mathrm{C}$ while irradiating at $150 \mathrm{~W}$ for $2 \mathrm{~min}$ (Scheme 1). Compounds 14, 16, 37, 48, 59, 62, 72 were obtained by heating at $60^{\circ} \mathrm{C}$ in DMF the corresponding indoles $\mathbf{9 8}-104$ with bis $(3,4,5$ trimethoxyphenyl)disulfide in the presence of sodium hydride. Acids 105 and 106 were similarly transformed into the corresponding sulfides $\mathbf{1 0 7}$ and 108, respectively. Acid 107 was then converted into ATI $\mathbf{2 8}$ by treatment with bromoethylamine hydrobromide in the presence of benzotriazol-1-yloxytris(dimethylamino)phosphonium hexa-fluorophosphate (BOP reagent) and triethylamine. Iodo derivative 108 was transformed into ATI 66 or 67 by heating at $110^{\circ} \mathrm{C}$ with an appropriate boronic acid pinacol ester in the presence of $1,1^{\prime}$ bis(diphenylphosphino)ferrocene]dichloro-palladium(II) complex with dichloromethane ( $\mathrm{Pd}(\mathrm{dppf})-\mathrm{Cl}_{2} \cdot \mathrm{CH}_{2} \mathrm{Cl}_{2}$ ) and potassium phosphate in anhydrous DMF. Organostannane reactions of 108 with 2-(triethylstannyl)- $1 H$-indole-1-carboxylic acid in the presence of bis(triphenylphosphine)palladium(II) dichloride $\left(\mathrm{PdCl}_{2}\left(\mathrm{PPh}_{3}\right)_{2}\right)$ in boiling ethanol for $48 \mathrm{~h}$ or 2-(tributylstannyl)oxazole, tris(dibenzylideneacetone)dipalladium(0) $\left(\mathrm{Pd}_{2}(\mathrm{dba})_{3}\right)$, and tricyclohexyl-phosphine $\mathrm{P}(\mathrm{Cy})_{3}$ in anhydrous DMF at $150{ }^{\circ} \mathrm{C}$ and $150 \mathrm{~W}$ afforded $\mathbf{6 8}$ or $\mathbf{3 1}$, respectively. The synthesis of 69 was performed by heating 108 at $100{ }^{\circ} \mathrm{C}$ in aqueous $1,4-$ dioxane in the presence of 2-benzofuranylboronic acid, palladium(II) acetate, and potassium phosphate. ATI 70 was synthesized by reaction of 108 with benzo[b]thien-2-ylboronic acid in the presence of tetrakis(triphenylphosphine)palladium $(0)\left(\mathrm{Pd}\left(\mathrm{PPh}_{3}\right)_{4}\right)$ and potassium carbonate in tetrahydrofuran at $60{ }^{\circ} \mathrm{C}$. Compound $\mathbf{7 1}$ was obtained from 108 and benzo $[b]$ thien-3-ylboronic acid in the presence of $\mathrm{Pd}_{2}(\mathrm{dba})_{3}, 2$-dicyclohexylphosphino-2', $6^{\prime}$-dimethoxybiphenyl (SPhos), and potassium phosphate in $n$-butanol at $100{ }^{\circ} \mathrm{C}$.

Ketones $7, \mathbf{1 5}, \mathbf{1 9}, \mathbf{5 4}, \mathbf{5 6}, \mathbf{5 8}$, and 60 were prepared by reaction of indoles $80,92-94,98$, 102, and $\mathbf{1 0 9}$ with methyl-magnesium bromide in the presence of anhydrous zinc chloride at $25^{\circ} \mathrm{C}$ and subsequent treatment with 3,4,5-trimethoxybenzoyl chloride and tin(IV) chloride (Scheme 2). Ketones 9, 21, 23, 25, 32, 35, 40, 42, 44, 51, 63, 74, 76, and 78 were obtained by reaction of the appropriate indoles 86-88, 91, 94-96, 103, and 110-114 with 3,4,5trimethoxybenzoyl chloride in the presence of anhydrous aluminum chloride. Reduction of ketones $9,21,23,25,35,51,60$, and 63 with borane-tetrahydrofuran complex solution in acetonitrile/methanol at $50{ }^{\circ} \mathrm{C}$ for $1 \mathrm{~h}$ afforded the corresponding methylene compounds $\mathbf{1 2}$, $22,24,26,36,52,61$, and 65 . Sodium hydroxide hydrolysis of 9,11 (obtained by sodium borohydride reduction of 9 ), or 12 in boiling methanol provided ketone $8, \mathbf{1 0}$, or $\mathbf{2 3}$, respectively. 
2-(1H-Pyrazol-4-yl)-1 $H$-indole (98) was synthesized by microwave reaction of 106 with 1Boc-pyrazole-4-boronic acid pinacol ester in the presence of $\mathrm{PdCl}_{2}\left(\mathrm{PPh}_{3}\right)_{2}, 1 \mathrm{M} \mathrm{Na}_{2} \mathrm{CO}_{3}$, and DMF in a closed vessel at $160{ }^{\circ} \mathrm{C}$ while irradiating at $250 \mathrm{~W}$ for $10 \mathrm{~min}$ (Scheme 3). Solvent-free reaction at $150^{\circ} \mathrm{C}$ of $\mathbf{1 0 6}$ with 1,2,4-triazole furnished 2-(1 H-1,2,4-triazol-1yl)-1 $H$-indole (81). 2-( $1 H$-Indol-2-yl)oxazole (114) was obtained as $\mathbf{3 1}$ by microwave reaction of 106 with 2-(tributylstannyl)oxazole in the presence of $\mathrm{Pd}_{2}(\mathrm{dba})_{3}$ and $\mathrm{P}(\mathrm{Cy})_{3}$ in anhydrous DMF at $150{ }^{\circ} \mathrm{C}$ and $150 \mathrm{~W}$. 2-(4,5-Dihydro- $1 H$-imidazol-2-yl)- $1 H$-indole (83) was prepared by treatment of $1 H$-indole-2-carbaldehyde $(\mathbf{1 1 5})^{26}$ with ethylenediamine and subsequent iodine oxidation in the presence of potassium carbonate. The 1-phenylethanones were converted into 2-bromo-1-phenylethanones 116-121 with bromine in dichloromethane and then transformed into the corresponding 2-phenylindoles 86-90, 101, and 102 by heating at $170{ }^{\circ} \mathrm{C}$ with aniline in $N, N$-dimethylaniline. Microwave reaction of 2-bromo-1(thiazol-2-yl)ethanone ${ }^{27}(\mathbf{1 1 6})$ with aniline in a closed vessel at $150{ }^{\circ} \mathrm{C}$ and $100 \mathrm{~W}$ for $1 \mathrm{~min}$ afforded 88. $o$-Toluidides 123-127 were transformed into the corresponding indoles 100, 104, and 95-97 with butyllithium in anhydrous tetrahydrofuran, while the mixture was warmed from -40 to $25^{\circ} \mathrm{C}$. Phenylhydrazono derivatives 128-133 underwent Fischer cyclization in polyphosphoric acid at $120^{\circ} \mathrm{C}$ to give indoles $85,91-94$, and 103.

\section{RESULTS AND DISCUSSION}

\section{Inhibition of Tubulin Polymerization}

The effects of ATIs 7-78 on tubulin polymerization in vitro are shown in Table 1. On the basis of our previous results, ${ }^{18,19}$ we synthesized these compounds to obtain structureactivity relationship (SAR) information regarding position 2 of the indole moiety and the sulfur bridging group. Several new ATIs $(\mathbf{7}, 8,10,15,16,18,20,21,23,38,39,41,43,49$, $\mathbf{5 3}, \mathbf{5 5}, \mathbf{6 8}-\mathbf{7 0}$, and 73) inhibited tubulin polymerization with $\mathrm{IC}_{50}$ of $1.0-2.0 \mu \mathrm{M}$, and six compounds $\left(\mathbf{1 4}, \mathbf{2 7}, \mathbf{3 7}, \mathbf{4 7}, \mathbf{5 7}\right.$, and 62) yielded $\mathrm{IC}_{50} \leq 1.0 \mu \mathrm{M}$, compared with $1.0 \mu \mathrm{M}$ obtained for CSA4 (2) and $3.2 \mu \mathrm{M}$ for colchicine (1).

For SAR studies regarding the sulfur bridging group, we synthesized 3-aroylindoles and several corresponding alcohol and methylene derivatives as potential bioisosteres of previously reported ATIs. ${ }^{19}$ As tubulin polymerization inhibitors, ketones $\mathbf{7}$ and $\mathbf{8}$ were less potent than their sulfur counterparts $\mathbf{3}$ and $\mathbf{4}$, respectively, as well as less active than the similar compounds 5 and $\mathbf{6}$. Similar results were were also obtained with ketones $\mathbf{2 1}, \mathbf{2 3}$, and 25 in comparison with the corresponding arylthioin-doles. ${ }^{19}$ Reduction of the ketone $\mathbf{8}$ to alcohol $\mathbf{1 0}$ resulted in an equipotent inhibitor of tubulin polymerization. Replacement of the sulfur bridging atom with a methylene group invariably caused a drop, sometimes dramatic, in inhibitory activity (cf. 4 with 13, 34 with 36, 28 with 30, 47 with 52, and 62 with 65 ).

Replacement of the pyrrol-3-yl group of $\mathbf{4}$ with a pyrazol-4-yl moiety provided ATI compound $14\left(\mathrm{IC}_{50}=0.92 \mu \mathrm{M}\right)$, which was more potent than the parent compound as an inhibitor of tubulin polymerization. This observation prompted the synthesis of new ATIs bearing a pyrazol-3-yl (16), imidazol-2-yl (17), imidazol-1-yl (18), 1,2,4-triazol-1-yl (20), thiazol-2-yl (27), or oxazol-2-yl (31) moiety at position 2 of the indole. With the exception of $\mathbf{1 7}$ and $\mathbf{3 1}$, these compounds potently inhibited tubulin polymerization with $\mathrm{IC}_{50}$ ranging from $0.96 \mu \mathrm{M}(\mathbf{2 7})$ to $1.3 \mu \mathrm{M}(\mathbf{1 8})$. Again, the ketones were less potent than the parent sulfur compounds (compare 14 with 15 , and 18 with 19).

Introduction of a methyl, methoxy, chlorine, or fluorine moiety at the para or meta position of a phenyl ring at indole position 2 also provided potent tubulin polymerization inhibitors (i.e., 37, 38, 41, 43, and 47), compared with the unsubstituted 34. Interestingly, ATI 49, bearing the 3-hydroxy-4-methoxyphenyl substitution pattern (the same as 2) at indole 
position 2, inhibited tubulin assembly with $\mathrm{IC}_{50}=1.1 \mu \mathrm{M}$, compared with $1.0 \mu \mathrm{M}$ for 2 . Introduction of a pyridin-2-yl $\left(\mathbf{5 3}, \mathrm{IC}_{50}=1.5 \mu \mathrm{M}\right)$, pyridin-3-yl $\left(\mathbf{5 5}, \mathrm{IC}_{50}=1.3 \mu \mathrm{M}\right)$, or pyridin-4-yl $\left(57, \mathrm{IC}_{50}=0.95 \mu \mathrm{M}\right)$ group resulted in potent inhibitors of tubulin assembly. The 2-naphthyl derivative $\mathbf{6 2}$ inhibited potently the tubulin polymerization $\left(\mathrm{IC}_{50}=1.0 \mu \mathrm{M}\right)$, but the 1-naphthyl derivative $\mathbf{5 9}$ was inactive. Compounds 68-70, bearing a benzofused heterocycl-2-yl substituent at position 2 of the indole, inhibited tubulin assembly with $\mathrm{IC}_{50}$ in the range of $1.5-1.8 \mu \mathrm{M}$.

Molecular modeling studies were consistent with the conclusion that the binding modes of these new ATIs in the colchicine site on tubulin were similar to those of previously reported ATIs ${ }^{19}$ (Supporting Information).

The new ATIs were also examined for potential inhibition of the binding of $\left[{ }^{3} \mathrm{H}\right]$ colchicine to tubulin (Table 1). Compounds 7, 8, 10, 14, 16, 18, 20, 21, 23, 27, 39, 41, 43, 55, and 57 inhibited the binding reaction by at least $70 \%$. Among them, $\mathbf{1 8}(92 \%), \mathbf{5 5}(88 \%)$, and $\mathbf{5 7}$ $(91 \%)$ were the strongest inhibitors of this binding reaction, compared with $\mathbf{2}(98 \%$ inhibition).

\section{Cell Growth Inhibition}

ATIs $7,8,10,14-16,18,20,21,23,25,34,39,40,47,55$, and 57 inhibited the growth of human MCF-7 nonmetastatic breast cancer epithelial cells with $\mathrm{IC}_{50} \leq 60 \mathrm{nM}$ (Table 1). Five compounds $(\mathbf{7}, \mathbf{1 8}, \mathbf{2 0}, \mathbf{5 5}$, and $\mathbf{5 7})$ had $\mathrm{IC}_{50} \leq 16 \mathrm{nM}$, with $18\left(\mathrm{IC}_{50}=1.0 \mathrm{nM}\right)$ being the most potent. As an inhibitor of MCF-7 cell growth, $\mathbf{1 8}$ was 5 and 13 times more potent than $\mathbf{1}$ and 2, respectively. Moreover, this ATI was 18-39 times more effective than the reference ATI compounds 3-6.

Many of the new agents were potent inhibitors of tubulin polymerization; however, compounds bearing the heterocycle nucleus at the indole position 2 were more effective as inhibitors of the growth of MCF-7 cells. ATI 49, having the same substitution pattern as 2, was only a relatively weak inhibitor of MCF-7 cell growth $\left(\mathrm{IC}_{50}=140 \mu \mathrm{M}\right)$. ATI derivatives $18\left(\mathrm{IC}_{50}=1.0 \mathrm{nM}\right)$ and $\mathbf{2 0}\left(\mathrm{IC}_{50}=11 \mathrm{nM}\right)$, bearing an imidazol-1-yl or 1,2,4triazol-1-yl nucleus, respectively, at indole position 2, were powerful inhibitors of the MCF-7 cell line. In contrast to 18, compounds $14\left(\mathrm{IC}_{50}=35 \mathrm{nM}\right), \mathbf{1 6}\left(\mathrm{IC}_{50}=50 \mathrm{nM}\right), \mathbf{1 7}$ $\left(\mathrm{IC}_{50}=270 \mathrm{nM}\right), 27\left(\mathrm{IC}_{50}=80 \mathrm{nM}\right)$, and $\mathbf{3 2}\left(\mathrm{IC}_{50}=270 \mathrm{nM}\right)$, each having two heteroatoms in the 2-heterocyclyl moiety, were less active inhibitors of the growth of this cell line.

Compounds $7,8,10,14,15,18,20,23,25,34,47,55$, and 57 were evaluated for growth inhibition of HeLa, HT29 (human colon adenocarcinoma), A549 (human lung carcinoma), HCT 116, and HCT 15 (human colon carcinoma cell lines) in comparison with $\mathbf{1 , 2}$, vinblastine (VBL), and paclitaxel (PTX) (Table 2). In terms of average $\mathrm{IC}_{50}$ for the five cell lines, the control compounds ranged from 17 to $40 \mathrm{nM}$ (ignoring the high value obtained with 2 in the A549 cells). The average value for 18,21 nM, was in the same range, and six other compounds $(\mathbf{7}, \mathbf{8}, \mathbf{1 4}, \mathbf{2 0}, \mathbf{5 5}$, and $\mathbf{5 7})$ had average $\mathrm{IC}_{50}$ values less than $100 \mathrm{nM}$. All these compounds had $\mathrm{IC}_{50}$ vlues of $35 \mathrm{nM}$ or less in the screening assay in the MCF7 cells.

\section{MDR Cell Lines}

Compounds 18, 20, 55, and $\mathbf{5 7}$ were compared with vinorelbine (VRB), VBL, PTX 1 and 2 in the ovarian carcinoma cell lines OVCAR-8 and its cognate P-glycoprotein (Pgp) overexpressing line NCI/ADR-RES and with the human uterine sarcoma cell line Messa and its cognate line MDR line Messa/Dx5 (Table 3). Except for 2, the standard agents were relatively inactive in both MDR lines. The four ATIs 18, 20, 55, and 57 closely ressembled 2 in having full activity in both MDR lines. 


\section{Effects on Cell Cycle Progression}

The most potent compound $\mathbf{1 8}$, as well as $\mathbf{5 7}$, was tested in dose-response experiments in comparison with $\mathbf{2}$ and VBL for effects on cell cycle distribution. HeLa cell cultures were treated with increasing concentrations of each compound, and $0.1 \%$ DMSO, the compound solvent, was used as a control. Representative images (DNA distribution by flow cytometry and wide-field microscopic images) of unfixed cultures taken after $24 \mathrm{~h}$ of treatment are shown in Figure 1A. Compound 57 at $100 \mathrm{nM}$ induced a significant increase in the proportion of cells in the G2/M phase and, presumably, in mitosis, based on their rounded morphology in the microscopic image. After treatment with $10 \mathrm{nM} \mathrm{18}$, a significant proportion of the cells had assumed the rounded shape characteristic of mitotic arrest, and at $50 \mathrm{nM}$ virtually the entire population was rounded and arrested in $\mathrm{G} 2 / \mathrm{M}$, based on DNA content.

To confirm the initial microscopic observations, treated cultures were incubated with propidium iodide and subjected to quantitative flow cytometric analysis of the cell cycle phase distribution. Typical cell cycle profiles of PI-stained cultures after $24 \mathrm{~h}$ of treatment are shown in Figure 1A, and average values calculated from three to five independent assays per compound are shown in Figure 1B. Both compounds 18 and 57 arrested cell cycle progression in the G2/M phases (4C DNA content) when used at $100 \mathrm{nM}$. Plots of the concentration of the tested ATI against the fraction of G2/M-arrested cells in treated cultures (Figure 1B) indicated that $\mathbf{1 8}$ was a potent inhibitor of cell cycle progression already at 20 $\mathrm{nM}$ and induced a significant proportion of cells (46\%) to arrest with a 4C DNA content, compared with $10.5 \%$ G2/M cells in control cultures. At higher concentrations, $\mathbf{1 8}$ progressively increased cell cycle arrest: at $50 \mathrm{nM}$, over $60 \%$ cells in treated cultures were in G2/M phase (Figure 1B), similar to the values obtained with both VBL and CSA-4 (2). The accumulation of cells with a replicated genome demonstrated that $\mathbf{1 8}$, like the control drugs, prevented or impaired mitotic cell division.

Compound 57 had somewhat milder effects on cell cycle progression (Figure 1B), compared with 18. Only about $48 \%$ of cells accumulated in the G2/M region with 57 at $50 \mathrm{nM}$; lower doses were virtually ineffective, with the proportion of G2/M cells essentially identical to the values observed in untretated controls. Only when the concentration was raised to 100 $\mathrm{nM}$ was the majority (65\%) of cells arrested in G2/M.

\section{Inhibition of Microtubule Assembly and Induction of Mitotic Arrest}

We analyzed cell cultures in dose-response experiments using fluorescence microscopy to gain information on the effects of $\mathbf{1 8}$ and $\mathbf{5 7}$ on cellular MTs. After treatment with increasing concentrations of $\mathbf{1 8}$ or $\mathbf{5 7}$ for $\mathbf{2 4} \mathrm{h}$, we stained treated cells for $\boldsymbol{a}$-tubulin, cyclin B1, and DAPI in order to discriminate between arrest in G2 interphase (i.e., interphase cells expressing cyclin B1, Figure 2A, left panel) or in prometaphase (Figure 2A, central panel). This is relevant because the molecular features of mitotic cell death are distinct, at least in part, from the classical apoptotic pathway induced in interphase. ${ }^{30}$ In some experiments, we also stained lamin B1 to visualize the integrity of the nuclear membrane and assess the stringency of mitotic arrest induced by ATI compounds. Indeed, cells with a defective mitotic apparatus do not necessarily arrest stably in prometaphase, but depending on the extent of MT damage, they may finally exit mitosis (mitotic slippage) with abnormal chromosome segregation and/or failed cell division giving rise to multi-nucleated cells (Figure 2A, right panel).

In dose-response experiments (Figure 2B, bottom panel), we found that $\mathbf{5 7}$ at 10 and $20 \mathrm{nM}$ did not affect mitotic progression (only $10 \%$ of all cells displayed mitotic properites, similar to the baseline frequency observed in control cells treated with DMSO). Although these 
concentrations induced many abnormal mitotic spindles (aberrantly shaped, multipolar, and/ or with sparse and rare MTs), cells were distributed in all mitotic stages, indicating that these abnormalities did not evoke a stringent spindle checkpoint, and cells progressed to divide. At 50 nM, 57 induced some mitotic arrest: $47 \%$ of all cells were arrested in prometaphase with reduced numbers of MTs. This arrest was not absolutely stringent, since $16 \%$ of cells in the culture were aberrant, multinucleated, postmitotic species. $100 \mathrm{nM} 57$ arrested almost $65 \%$ of cells stably in prometaphase with completely absent MTs and condensed chromosomes. Virtually no mitotic slippage was observed with this concentration. At $200 \mathrm{nM}$, the mitotic index decreased while the fraction of G2 interphases correspondingly increased, indicating such a strong effect on interphase MTs that cells were prevented from entering mitosis altogether. ${ }^{31}$ Indeed, a similar decrease in mitotic arrest, with a corresponding increase in G2 cells, is observed at high concentrations of $\mathbf{2}$ or VBL.

Compound 18 showed the highest effectiveness, in terms of inducing mitotic arrest at $50 \mathrm{nM}$ in HeLa cell cultures (Figure 2B, top panel). At this concentration, 18 induced effective mitotic arrest in prometaphase with totally absent MTs and no significant mitotic slippage. ATI 18 blocked mitotic progression even at lower concentration $(20 \mathrm{nM})$, yet the arrest was not fully sustained with this concentration, and some cells slipped through mitosis with a defective mitotic apparatus, generating multi-nucleated cells (18\% of all cells). At $100 \mathrm{nM}$, 18 prevented M entry, suggesting an excessively strong effect on interphase MTs. Together, these data indicate that $\mathbf{1 8}$ is a strong inhibtior of MT polymerization and induced effective mitotic arrest at $50 \mathrm{nM}$, similar to $\mathbf{2}$ or VBL, whereas $\mathbf{5 7}$ induced a durable mitotic block when used at $100 \mathrm{nM}$.

\section{Induction of Mitotic Cell Death}

We examined whether the new ATIs $\mathbf{1 8}$ and 57, in addition to arresting mitosis, also triggered cell death. HeLa cell cultures were treated for $24 \mathrm{~h}$ as described above, and they were then incubated with annexin $\mathrm{V}$ in the absence of permeabilization in order to detect apoptotic cells with a damaged plasma membrane. They were then analyzed by flow cytometry (Figure 3A). Significant cell death was detected in cultures exposed to 18 (50 and $100 \mathrm{nM})$ or $\mathbf{5 7}(100 \mathrm{nM})$ concentrations at which the cell populations displayed significant mitotic arrest (Figures 1 and 2). Biparametric analysis of annexin $\mathrm{V}$ reactivity versus genomic DNA content revealed that necrosis was negligible (Figure 3B). These data therefore show that both $\mathbf{1 8}$ and $\mathbf{5 7}$ induce mitotic cell death.

\section{Loss of Mitochondrial Potential}

We evaluated the loss of mitochondrial potential $\left(\Delta \Psi_{\mathrm{m}}\right)$, a hallmark for early induction of cellular apoptosis, ${ }^{32}$ to elucidate further whether compounds 18 and $\mathbf{5 7}$ would induce apoptosis or necrotic death. The loss of $\Delta \Psi_{\mathrm{m}}$ was indicated by a decrease in red 5,5',6,6'tetrachloro-1, $1^{\prime} 3,3^{\prime}$-tetraethylbenzimidazoylcarbocyanine iodide (JC-1) aggregates, accompanied by a concomitant accumulation in green JC-1 monomers. The collapse in $\Delta \Psi_{\mathrm{m}}$, as measured as reduction in the red/green fluorescence ratio, was assessed after cell treatment with 18 or $\mathbf{5 7}$. As shown in Figure 4, after a $24 \mathrm{~h}$ treatment, $\mathbf{5 7}$ was able to induce a more significant reduction in $\Delta \Psi_{\mathrm{m}}$ than was $\mathbf{1 8}$. The standard compound carbonyl cyanide $m$-chlorophenylhydrazone (CCCP) caused about $50 \%$ potential dissipation at $30 \mu \mathrm{M}$.

\section{ROS Generation}

Mitochondria are an important intra-cellular source of reactive oxygen species (ROS). ${ }^{33} \mathrm{We}$ measured the ability of compounds $\mathbf{1 8}$ and $\mathbf{5 7}$ to generate ROS in U87MG cells, using hydrogen peroxide specific probe 6-carboxy-2', $7^{\prime}$-lichlorodihydrofiuorescein diacetate (DCFH2-DA). 
The $\mathrm{IC}_{50}$ values of compounds $\mathbf{1 8}$ and $\mathbf{5 7}$ in U87MG (human glioblastoma) cell growth/ survival after a $24 \mathrm{~h}$ treatment were determined. Compounds $\mathbf{1 8}$ and $\mathbf{5 7}$ showed dosedependent inhibitory effects on cell survival, with compound 18 having $\mathrm{IC}_{50}=85 \pm 8 \mathrm{nM}$ and 57 having $\mathrm{IC}_{50}=103 \pm 9 \mathrm{nM}$. Compound-treated cells showed an increase in the percentage of cell death compared with control cells, reaching statistical significance at 100 $\mathrm{nM}$.

According to the observed loss of mitochondrial potential, both compounds $\mathbf{1 8}$ and $\mathbf{5 7}$ were able to increase ROS levels. The effect was not detectable immediately, but it reached statistical significance after a $1 \mathrm{~h}$ treatment (fluorescence intensity with respect to control: $\mathbf{1 8}, 216 \% ; \mathbf{5 7}, 254 \%$; both greater than the value of $208 \%$ obtained with the usual standard, hydrogen peroxide (Figure 5)).

\section{In Vivo Vascular Disrupting Effects}

In preclinical development of new anticancer drugs such as vascular disrupting agents (VDAs), in vivo validation proved to be an indispensible step in the bench-to-bedside chain, where animal models with tumor growth in visceral organs could better simulate clinical scenarios in comparison with the mostly applied subcutaneous implants. ${ }^{34}$ Magnetic resonance imaging (MRI) has greatly facilitated in vivo noninvasive assessment of tumoricidal effects on deeply seeded lesions in the animal models. ${ }^{35}$ Consequently, we were able to observe that at the same intravenous dosage of $15 \mathrm{mg} / \mathrm{kg}$, both compounds $\mathbf{1 8}$ and $\mathbf{5 7}$ exerted initial vascular disrupting effects in the applied tumor model of liver rhabdomyosarcomas. The observed tumor vascular shutdown effect seems stronger and lasts longer with $18\left(\mathrm{~B}^{\prime}\right.$ and $\left.\mathrm{C}^{\prime}\right)$ compared to $\mathbf{5 7}$ (B and $\mathrm{C}$ ). The reason for such different effects will be further elucidated (Figure 6).

\section{Metabolic Stability}

Compounds 18, 20, 55, and 57 were examined in a microsomal stability assay in comparison with 7-ethoxycoumarin and propranolol as control compounds, using both human and mouse liver microsomes, to estimate compound stability to phase I oxidative metabolism (Table 4). Compound $\mathbf{1 8}$ showed the highest metabolic stability with $48.6 \%$ and $10.3 \%$ remaining after $30 \mathrm{~min}$ in human and mouse liver microsomes, respectively. Compounds $\mathbf{2 0}$, $\mathbf{5 5}$, and $\mathbf{5 7}$ showed medium metabolic stability with human liver microsomes and low metabolic stability with mouse liver microsomes (relative stabilities are defined in a Table 4 footnote).

\section{Aqueous Solubility}

The solubility in aqueous $\mathrm{pH} 7.4$ buffer of compounds $\mathbf{1 8}, \mathbf{2 0}, \mathbf{5 5}$, and $\mathbf{5 7}$ was measured in a high throughput screening solubility assay. The solubility of compound 18 was $64.5 \mu \mathrm{M}$, while under the same conditions compounds $20(4 \mu \mathrm{M}), \mathbf{5 5}(20.5 \mu \mathrm{M})$, and $57(8 \mu \mathrm{M})$ showed lower solubility (Table 4 ). The higher solubility of compound $\mathbf{1 8}$ could explain its greater activity as a cell growth inhibitor (see Tables 1 and 2), although these compounds were comparable inhibitors of tubulin assembly in the biochemical assays.

\section{Caco-2 Cell Permeability}

The intestinal permeability of compounds $18, \mathbf{2 0}, \mathbf{5 5}$, and $\mathbf{5 7}$ was evaluated in the human Caco-2 model in comparison with caffeine (high permeability) and cimetidine (low permeability, Pgp substrate). The apparent permeability $\left(P_{\text {app }}\right)$ of $\mathbf{1 8}, \mathbf{2 0}, \mathbf{5 5}$, or 57 from the A (apical) to B (basolateral) side together with B to A was measured in order to predict the absorption from the lumen of the gut and potential efflux phenomena. Compound $\mathbf{2 0}$ showed the highest permeability in both $\mathrm{A} \rightarrow \mathrm{B}\left(P_{\mathrm{app}}=132.5 \mathrm{~nm} / \mathrm{s}\right)$ and B $\rightarrow \mathrm{A}\left(P_{\mathrm{app}}=133.2 \mathrm{~nm} / \mathrm{s}\right)$ 
directions. Compounds $\mathbf{1 8}, \mathbf{5 5}$, and $\mathbf{5 7}$ showed medium-high permeability (Table 5). None of the compounds tested showed efflux phenomena (i.e., ratio of $P_{\text {app }}(\mathrm{B} \rightarrow \mathrm{A})$ to $P_{\text {app }}(\mathrm{A} \rightarrow \mathrm{B})$ of $<2$ ).

\section{CYP450 Inhibition}

To assess further the safety of compounds $\mathbf{1 8}, \mathbf{2 0}, \mathbf{5 5}$, and $\mathbf{5 7}$, we carried out a CYP inhibition assay. CYP inhibition can cause drug-drug interactions by increasing concentrations to other drugs to toxic levels. The inhibition of the enzymatic activity of human cloned P450 isoforms CYP1A2, CYP2C19, CYP2C9, CYP2D6, and CYP3A4 was measured using specific substrates for each isoform that produced a fluorescent metabolite upon CYP metabolism. Compounds 18, 20, 55, and 57 at $1 \mu \mathrm{M}$ were weak inhibitors of the isoforms CYP1A2 and CYP2D6. For these isoforms, inhibition was $<50 \%$, ranging from $<5 \%$ (20 in CYP1A2 and CYP2D6 isoforms) to 43\% (57 in CYP2D6 isoform). The isoform CYP3A4 was inhibited by $\mathbf{2 0}, \mathbf{5 5}$, and $\mathbf{7 7}$ by $51 \%, 76 \%$, and $57 \%$, respectively; CYP2C19 and CYP2C9 were inhibited to a greater extent by 18, 20, 55, and 57.

\section{CONCLUSION}

We synthesized 71 new arylthioindole/aroylindole compounds as potential anticancer agents having different (hetero)cyclic substituents at indole position 2. Several new ATIs inhibited tubulin polymerization with $\mathrm{IC}_{50}$ ranging from $<1.0$ to $2.0 \mu \mathrm{M}$, and six compounds yielded submicromolar $\mathrm{IC}_{50}$. These compounds inhibited the growth of human MCF-7 cells, and five of them inhibited the growth of these cells with low nanomolar $\mathrm{IC}_{50}$. ATI 18 strongly inhibited MCF-7 cell growth $\left(\mathrm{IC}_{50}=1.0 \mathrm{nM}\right)$ and was uniformly active in a larger panel of cancer cells. Compound $\mathbf{1 8}$ was superior to reference compounds $\mathbf{1}$ and $\mathbf{2}$ and ATIs 3-6. ${ }^{19}$ ATIs 18, 20, 55, and 57 were more potent than 1, 2, VRB, VBL, and PTX in two MDR cell lines, the Pgp overexpressing NCI-ADR-RES line and the Messa/Dx5 line. Compounds 18 and $\mathbf{5 7}$ arrested cell cycle progression at the G2/M phase. Compound $\mathbf{1 8}$ was a potent inhibitor of cell cycle progression at $20 \mathrm{nM}$, strongly inhibited MT polymerization, and induced effective mitotic arrest and cell death, while $\mathbf{5 7}$ showed somewhat weaker effects. Compounds 18 and 57 elicited dose- and time-dependent inhibition of U87MG cell growth and induced mitochondrial membrane potential collapse, a hallmark of early apoptosis. The compounds also evoked significant ROS generation in GBM cells. At $15 \mathrm{mg} / \mathrm{kg}$ intravenous dosage, both compounds $\mathbf{1 8}$ and $\mathbf{5 7}$ exerted initial vascular disrupting effects in a tumor model of liver rhabdomyosarcomas. In a microsomal stability assay, compound $\mathbf{1 8}$ showed the highest metabolic stability with both human and mouse liver microsomes of all the ATIs examined. The higher solubility in aqueous $\mathrm{pH} 7.4$ buffer of compound $\mathbf{1 8}(64.5 \mu \mathrm{M})$ could explain its greater inhibitory effects on cell growth $\left(\mathrm{MCF}-7\right.$ cells, $\left.\mathrm{IC}_{50}=1.0 \mathrm{nM}\right)$ among compounds with comparable inhibitory effects on tubulin assembly. In conclusion, we succeeded in improving the ATI class of cancer agents through the synthesis of new compounds. Compounds $\mathbf{1 8}$ and $\mathbf{5 7}$ proved to be superior to $\mathbf{5}$, the reference ATI of the previous series, ${ }^{19}$ in the whole cancer cell panel, including two MDR cell lines. Derivative 18 showed higher metabolic stability than $\mathbf{5}$ in human liver microsomes (48.6\% remaining), showed the highest water solubility, and displayed medium-high Caco-2 cell permeability. The present results highlight the therapeutic potential of the ATI class as anticancer agents and prompt their further development.

\section{EXPERIMENTAL SECTION}

Microwave-assisted reactions were performed on a CEM Discover SP single mode reactor, controlling the instrument settings with PC-running CEM Synergy 1.49 software. Closed vessel experiments were carried out in capped microwave-dedicated vials $(10 \mathrm{~mL})$ with 
cylindrical stirring bar (length $8 \mathrm{~mm}$, diameter $3 \mathrm{~mm}$ ). Open vessel experiments were carried out in $100 \mathrm{~mL}$ round-bottom flasks equipped with a Dimroth reflux condenser and a cylindrical stirring bar (length $20 \mathrm{~mm}$, diameter $6 \mathrm{~mm}$ ). Stirring, temperature, irradiation power, maximum pressure $\left(P_{\max }\right)$, PowerMAX (simultaneous cooling-while-heating), ActiVent (simultaneous venting-while-heating), and ramp and hold times were set as indicated. Temperature of the reaction was monitored by a built-in infrared sensor. After completion of the reaction, the mixture was cooled to $25^{\circ} \mathrm{C}$ via air-jet cooling. Melting points $(\mathrm{mp})$ were determined on a Stuart Scientific SMP1 apparatus and are uncorrected. Infrared spectra (IR) were run on a Perkin-Elmer SpectrumOne FT-ATR spectrophotometer. Band position and absorption ranges are given in $\mathrm{cm}^{-1}$. Proton nuclear magnetic resonance $\left({ }^{1} \mathrm{H}\right.$ NMR) spectra were recorded on a Bruker $400 \mathrm{MHz}$ FT spectrometer in the indicated solvent and corresponding fid files processed by MestreLab Research S.L. MestreReNova 6.2.1-769 software. Chemical shifts are expressed in $\delta$ units (ppm) from tetramethylsilane. Column chromatography was performed on columns packed with alumina from Merck (70230 mesh) or silica gel from Macherey-Nagel (70-230 mesh). Aluminum oxide thin layer chromatography (TLC) cards from Fluka (aluminum oxide precoated aluminum cards with fluorescent indicator visualizable at $254 \mathrm{~nm}$ ) and silica gel TLC cards from Macherey-Nagel (silica gel precoated aluminum cards with fluorescent indicator visualizable at $254 \mathrm{~nm}$ ) were used for TLC. Developed plates were visualized by a Spectroline ENF 260C/FE UV apparatus. Organic solutions were dried over anhydrous $\mathrm{Na}_{2} \mathrm{SO}_{4}$. Evaporation of the solvents was carried out on a Buchi rotavapor R-210 equipped with a Buchi V-850 vacuum controller and a Buchi V-700 or V-710 vacuum pump. All reagents and solvents are commercially available and were used as purchased, without further purification. Elemental analyses of the compounds were found within $\pm 0.4 \%$ of the theoretical values. The purity of tested compounds was $>95 \%$.

2-(1H-Imidazol-1-yl)-1 $H$-indole (80), ${ }^{21}$ 2-(1 $H$-pyrazol-3-yl)- $1 H$-indole (99), ${ }^{22}$ 2-iodo- $1 H$ indole (106), ${ }^{23}$ 2-(1 H-pyrrol-2-yl)- $1 H$-indole (109), ${ }^{25}$ 2-(1-(phenylsulfonyl)- $1 H$-pyrrol-3yl)- $1 H$-indole (110), ${ }^{19}$ 2-(furan-2-yl)- $1 H$-indole (111), ${ }^{19}$ 2-(furan-3-yl)- $1 H$-indole (112), ${ }^{19}$ $1 H$-indole-2-carbaldehyde (115), ${ }^{26}$ 2-(1-(2-phenylhydrazono)ethyl)-pyridine (130), ${ }^{29} 3$-(1(2-phenylhydrazono)ethyl)pyridine (131) ${ }^{29}$ 4-(1-(2-phenylhydrazono)ethyl)pyridine (132) ${ }^{29}$ and 1-(1-(naphthalen-2-yl)ethylidene)-2-phenylhydrazine $(\mathbf{1 3 3})^{29}$ were synthesized according to the indicated literature. 2-Phenyl- $1 H$-indole (84), $1 H$-indole-2-carboxylic acid (105), 2-bromo-1-(2-methoxyphenyl)ethanone (119), and 2-bromo-1-(3-

methoxyphenyl)ethanone (120) were purchased from Sigma-Aldrich.

\section{Arylthio- $1 \mathrm{H}$-indoles. General Procedure for the Preparation of Compounds 17, 18, 20, 27, $33,34,38,39,41,43,45-47,53,55,57,73,75$, and 77 . Example: 2-(1H-Imidazol-2-yl)-3- ((3,4,5-trimethoxyphenyl)thio)-1 $\mathrm{H}$-indole (17)}

2-(1H-Imidazol-2-yl)-1 $H$-indole (79) (0.044 g, $0.00024 \mathrm{~mol})$ was added to a suspension of $\mathrm{NaH}(0.014 \mathrm{~g}, 0.00036 \mathrm{~mol}, 60 \%$ dispersion in mineral oil $)$ in anhydrous DMF $(2 \mathrm{~mL})$. After $10 \mathrm{~min}$, bis(3,4,5-trimethoxyphenyl)disulfide $(0.1 \mathrm{~g}, 0.000264 \mathrm{~mol})$ was added, and the reaction mixture was placed into the microwave cavity (closed vessel mode, $P_{\max }=250$ psi). A starting microwave irradiation of $150 \mathrm{~W}$ was used, the temperature being ramped from 25 to $110^{\circ} \mathrm{C}$ while stirring. Once $110^{\circ} \mathrm{C}$ was reached, taking about $1 \mathrm{~min}$, the reaction mixture was held at this temperature for 2 min. The reaction mixture was quenched on crushed ice and extracted with ethyl acetate. The organic layer was washed with brine, dried, and filtered. Removal of the solvent gave a residue that was purified by column chromatography (silica gel, ethyl acetate $/ n$-hexane $=7: 3$ as eluent) to furnish $\mathbf{1 7}(0.02 \mathrm{~g}$, $22 \%), \mathrm{mp} 135-140{ }^{\circ} \mathrm{C}$ (from ethanol). ${ }^{1} \mathrm{H} \mathrm{NMR}\left(\mathrm{CDCl}_{3}\right): \delta 3.62(\mathrm{~s}, 6 \mathrm{H}), 3.76(\mathrm{~s}, 3 \mathrm{H}), 6.38$ (s, 2H), 7.14-7.26 (m, 4H), 7.36-7.38 (m, 1H), $7.63(\mathrm{~d}, J=7.9 \mathrm{~Hz}, 1 \mathrm{H}), 11.01$ (broad s, 
disappeared on treatment with $\left.\mathrm{D}_{2} \mathrm{O}, 1 \mathrm{H}\right), 11.58 \mathrm{ppm}$ (broad s, disappeared on treatment with $\left.\mathrm{D}_{2} \mathrm{O}, 1 \mathrm{H}\right)$. IR: $v 2923,3345 \mathrm{~cm}^{-1}$. Anal. $\left(\mathrm{C}_{20} \mathrm{H}_{19} \mathrm{~N}_{3} \mathrm{O}_{3} \mathrm{~S}(381.45)\right) \mathrm{C}, \mathrm{H}, \mathrm{N}, \mathrm{S}$.

2-(1 H-Imidazol-1-yl)-3-((3,4,5-trimethoxyphenyl)thio)-1 $\boldsymbol{H}$-indole (18)-18 was synthesized similarly to $\mathbf{1 7}$, starting from $\mathbf{8 0} .^{21}$ Yield $77 \%$, mp $193-197{ }^{\circ} \mathrm{C}$ (from ethanol). ${ }^{1} \mathrm{H}$ NMR (DMSO- $\left.d_{6}\right): \delta 3.57(\mathrm{~s}, 6 \mathrm{H}), 3.58(\mathrm{~s}, 3 \mathrm{H}), 6.31(\mathrm{~s}, 2 \mathrm{H}), 7.17-7.21(\mathrm{~m}$, 2H), 7.26-7.30 (m, 1H), 7.49-7.55 (m, 2H), 7.68-7.69 (m, 1H), 8.14-7.15 (m, 1H), 12.23 ppm (broad s, disappeared on treatment with $\mathrm{D}_{2} \mathrm{O}, 1 \mathrm{H}$ ). ${ }^{13} \mathrm{C}$ NMR (DMSO- $d_{6}$ ): $\delta 56.33$, 60.48, 91.97, 103.95, 112.62, 119.17, 120.49, 121.66, 123.68, 129.27, 129.77, 132.91, 133.81, 136.2, 136.23, 137.78, 153.79 ppm. IR: $v 2930 \mathrm{~cm}^{-1}$. Anal. $\left(\mathrm{C}_{20} \mathrm{H}_{19} \mathrm{~N}_{3} \mathrm{O}_{3} \mathrm{~S}\right.$ (381.45)) C, H, N, S.

2-(1 H-1,2,4-Triazol-1-yl)-3-((3,4,5-trimethoxyphenyl)thio)-1 $H$-indole (20)-20 was synthesized similarly to $\mathbf{1 7}$, starting from 81 . Yield $34 \%$, mp $140-145{ }^{\circ} \mathrm{C}$ (from ethanol). ${ }^{1} \mathrm{H}$ NMR (DMSO- $\left.d_{6}\right): \delta 3.58(\mathrm{~s}, 9 \mathrm{H}), 6.37(\mathrm{~s}, 2 \mathrm{H}), 7.21(\mathrm{t}, J=7.7 \mathrm{~Hz}, 1 \mathrm{H}), 7.31(\mathrm{t}, J=8.0 \mathrm{~Hz}$, $1 \mathrm{H}), 7.52-7.58(\mathrm{~m}, 2 \mathrm{H}), 8.41(\mathrm{~s}, 1 \mathrm{H}), 9.12(\mathrm{~s}, 1 \mathrm{H}), 12.80 \mathrm{ppm}$ (broad s, disappeared on treatment with $\mathrm{D}_{2} \mathrm{O}, 1 \mathrm{H}$ ). ${ }^{13} \mathrm{C}$ NMR (DMSO- $d_{6}$ ): $\delta 56.32,60.47,93.24,104.45,113.01$, 119.50, 121.78, 124.14, 128.78, 132.15, 134.11, 135.32, 136.38, 145.67, 153.04, 153.75 ppm. IR: $v 3354 \mathrm{~cm}^{-1}$. Anal. $\left(\mathrm{C}_{19} \mathrm{H}_{18} \mathrm{~N}_{4} \mathrm{O}_{3} \mathrm{~S}(382.44)\right) \mathrm{C}, \mathrm{H}, \mathrm{N}, \mathrm{S}$.

2-(3-((3,4,5-Trimethoxyphenyl)thio)-1 $\boldsymbol{H}$-indol-2-yl)thiazole (27)-27 was synthesized similarly to $\mathbf{1 7}$, starting from $\mathbf{8 2}$. Yield $10 \%, \mathrm{mp} 160-165{ }^{\circ} \mathrm{C}$ (from ethanol). ${ }^{1} \mathrm{H}$ $\operatorname{NMR}\left(\mathrm{CDCl}_{3}\right): \delta 3.65(\mathrm{~s}, 6 \mathrm{H}), 3.77(\mathrm{~s}, 3 \mathrm{H}), 6.41(\mathrm{~s}, 2 \mathrm{H}), 7.19-7.23(\mathrm{~m}, 1 \mathrm{H}), 7.31-7.35(\mathrm{~m}$, $1 \mathrm{H}), 7.43-7.45(\mathrm{~m}, 2 \mathrm{H}), 7.72(\mathrm{dd}, J=0.8$ and $7.9 \mathrm{~Hz}, 1 \mathrm{H}), 7.90(\mathrm{~d}, J=3.2 \mathrm{~Hz}, 1 \mathrm{H}), 9.87$ ppm (broad s, disappeared on treatment with $\mathrm{D}_{2} \mathrm{O}, 1 \mathrm{H}$ ). IR: $v 3339 \mathrm{~cm}^{-1}$. Anal. $\left(\mathrm{C}_{20} \mathrm{H}_{18} \mathrm{~N}_{2} \mathrm{O}_{3} \mathrm{~S}_{2}(379.47)\right) \mathrm{C}, \mathrm{H}, \mathrm{N}, \mathrm{S}$.

\section{2-(4,5-Dihydro-1 $\mathrm{H}$-imidazol-2-yl)-3-((3,4,5-trimethoxyphenyl)-thio)-1 $\mathrm{H}$-indole} (33)-33 was synthesized similarly to $\mathbf{1 7}$, starting from $\mathbf{8 3}$. Yield $8 \%, \mathrm{mp} 150-155{ }^{\circ} \mathrm{C}$ (from ethanol). ${ }^{1} \mathrm{H} \mathrm{NMR}\left(\mathrm{CDCl}_{3}\right): \delta 3.64(\mathrm{~s}, 6 \mathrm{H}), 3.76(\mathrm{~s}, 3 \mathrm{H}), 3.92(\mathrm{~m}, 4 \mathrm{H}), 6.33(\mathrm{~s}, 2 \mathrm{H})$, $7.15(\mathrm{t}, J=7.1 \mathrm{~Hz}, 1 \mathrm{H}), 7.32(\mathrm{t}, J=7.0 \mathrm{~Hz}, 1 \mathrm{H}), 7.49(\mathrm{~d}, J=8.3 \mathrm{~Hz}, 1 \mathrm{H}), 7.61(\mathrm{~d}, J=8.1$ $\mathrm{Hz}, 1 \mathrm{H}$ ), 8.31 (broad s, disappeared on treatment with $\mathrm{D}_{2} \mathrm{O}, 1 \mathrm{H}$ ), $12.10 \mathrm{ppm}$ (broad s, disappeared on treatment with $\left.\mathrm{D}_{2} \mathrm{O}, 1 \mathrm{H}\right)$. IR: $v 2852,2921 \mathrm{~cm}^{-1}$. Anal. $\left(\mathrm{C}_{20} \mathrm{H}_{21} \mathrm{~N}_{3} \mathrm{O}_{3} \mathrm{~S}\right.$ (383.46)) C, H, N, S.

2-Phenyl-3-((3,4,5-trimethoxyphenyl)thio)-1 H-indole (34)-34 was synthesized similarly to $\mathbf{1 7}$, starting from 84 . Yield $3 \%, \mathrm{mp} 158-160{ }^{\circ} \mathrm{C}$ (from ethanol). ${ }^{1} \mathrm{H}$ NMR $\left(\mathrm{CDCl}_{3}\right): \delta 3.64(\mathrm{~s}, 6 \mathrm{H}), 3.78(\mathrm{~s}, 3 \mathrm{H}), 6.37(\mathrm{~s}, 2 \mathrm{H}), 7.18-7.22(\mathrm{~m}, 1 \mathrm{H}), 7.27-7.30(\mathrm{~m}, 2 \mathrm{H})$, $7.41-7.50(\mathrm{~m}, 3 \mathrm{H}), 7.69(\mathrm{~d}, J=7.9 \mathrm{~Hz}, 1 \mathrm{H}), 7.80-7.82(\mathrm{~m}, 2 \mathrm{H}), 8.56 \mathrm{ppm}$ (broad s, disappeared on treatment with $\left.\mathrm{D}_{2} \mathrm{O}, 1 \mathrm{H}\right)$. IR: $v 3329 \mathrm{~cm}^{-1}$. Anal. $\left(\mathrm{C}_{23} \mathrm{H}_{21} \mathrm{NO}_{3} \mathrm{~S}(391.48)\right)$ $\mathrm{C}, \mathrm{H}, \mathrm{N}, \mathrm{S}$.

\section{2-(4-Chlorophenyl)-3-((3,4,5-trimethoxyphenyl)thio)-1 $\boldsymbol{H}$-indole (38)-38 was}

synthesized similarly to $\mathbf{1 7}$, starting from $\mathbf{8 5}$. Yield $11 \%$, mp $190-195{ }^{\circ} \mathrm{C}$ (from ethanol). ${ }^{1} \mathrm{H}$ $\operatorname{NMR}\left(\mathrm{CDCl}_{3}\right): \delta 3.64(\mathrm{~s}, 6 \mathrm{H}), 3.78(\mathrm{~s}, 3 \mathrm{H}), 6.34(\mathrm{~s}, 2 \mathrm{H}), 7.19-7.23(\mathrm{~m}, 1 \mathrm{H}), 7.29-7.31(\mathrm{~m}$, $1 \mathrm{H}), 7.43-7.46(\mathrm{~m}, 3 \mathrm{H}), 7.68-7.70(\mathrm{~m}, 1 \mathrm{H}), 7.72-7.75(\mathrm{~m}, 2 \mathrm{H}), 8.59 \mathrm{ppm}$. (broad s, disappeared on treatment with $\left.\mathrm{D}_{2} \mathrm{O}, 1 \mathrm{H}\right)$. IR: $v 3335 \mathrm{~cm}^{-1}$. Anal. $\left(\mathrm{C}_{23} \mathrm{H}_{20} \mathrm{ClNO}_{3} \mathrm{~S}(425.93)\right)$ $\mathrm{C}, \mathrm{H}, \mathrm{N}, \mathrm{Cl}, \mathrm{S}$.

2-(2-Fluorophenyl)-3-((3,4,5-trimethoxyphenyl)thio)-1 $\mathrm{H}$-indole (39)-39 was synthesized similarly to $\mathbf{1 7}$, starting from $\mathbf{8 6}$. Yield $39 \% \mathrm{mp} 175-180{ }^{\circ} \mathrm{C}$ (from toluene). ${ }^{1} \mathrm{H}$ NMR (DMSO- $\left.d_{6}\right): \delta 3.54(\mathrm{~s}, 6 \mathrm{H}), 3.56(\mathrm{~s}, 3 \mathrm{H}), 6.26(\mathrm{~s}, 2 \mathrm{H}), 7.13(\mathrm{t}, J=7.6 \mathrm{~Hz}, 1 \mathrm{H}), 7.24(\mathrm{t}$, 
$J=7.8 \mathrm{~Hz}, 1 \mathrm{H}), 7.33-7.42(\mathrm{~m}, 2 \mathrm{H}), 7.49-7.54(\mathrm{~m}, 3 \mathrm{H}), 7.65(\mathrm{t}, J=7.5 \mathrm{~Hz}, 1 \mathrm{H}), 12.07 \mathrm{ppm}$ (broad s, disappeared on treatment with $\left.\mathrm{D}_{2} \mathrm{O}, 1 \mathrm{H}\right)$. IR: $v 3342 \mathrm{~cm}^{-1}$. Anal. $\left(\mathrm{C}_{23} \mathrm{H}_{20} \mathrm{FNO}_{3} \mathrm{~S}\right.$ (409.47)) C, H, N, F, S.

2-(3-Fluorophenyl)-3-((3,4,5-trimethoxyphenyl)thio)-1 $\mathrm{H}$-indole (41)-41 was synthesized similarly to $\mathbf{1 7}$, starting from $\mathbf{8 7}$. Yield $65 \%$, mp $180-185{ }^{\circ} \mathrm{C}$ (from toluene). ${ }^{1} \mathrm{H}$ NMR (DMSO- $\left.d_{6}\right): \delta 3.59(\mathrm{~s}, 3 \mathrm{H}), 3.64(\mathrm{~s}, 6 \mathrm{H}), 6.79(\mathrm{~s}, 2 \mathrm{H}), 7.07-7.35(\mathrm{~m}, 5 \mathrm{H}), 7.45-7.48$ $(\mathrm{m}, 1 \mathrm{H}), 7.52(\mathrm{~d}, J=8.0 \mathrm{~Hz}, 1 \mathrm{H}), 7.95(\mathrm{~d}, J=7.8 \mathrm{~Hz}, 1 \mathrm{H}), 12.27$ (broad s, disappeared on treatment with $\left.\mathrm{D}_{2} \mathrm{O}, 1 \mathrm{H}\right)$. IR: $v 1571,3187 \mathrm{~cm}^{-1}$. Anal. $\left(\mathrm{C}_{24} \mathrm{H}_{20} \mathrm{FNO}_{4}(405.42)\right) \mathrm{C}, \mathrm{H}, \mathrm{N}, \mathrm{F}$, S.

2-(4-Fluorophenyl)-3-((3,4,5-trimethoxyphenyl)thio)-1 $\boldsymbol{H}$-indole (43)-43 was synthesized similarly to $\mathbf{1 7}$, starting from $\mathbf{8 8}$. Yield $37 \%, \mathrm{mp} 170-175^{\circ} \mathrm{C}$ (from toluene). ${ }^{1} \mathrm{H}$ NMR (DMSO- $\left.d_{6}\right): \delta 3.53(\mathrm{~s}, 6 \mathrm{H}), 3.57(\mathrm{~s}, 3 \mathrm{H}), 6.30(\mathrm{~s}, 2 \mathrm{H}), 7.12(\mathrm{t}, J=7.0 \mathrm{~Hz}, 1 \mathrm{H}), 7.23(\mathrm{t}$, $J=7.4 \mathrm{~Hz}, 1 \mathrm{H}), 7.38(\mathrm{t}, J=8.9 \mathrm{~Hz}, 2 \mathrm{H}), 7.49(\mathrm{t}, J=7.2 \mathrm{~Hz}, 2 \mathrm{H}), 7.90-7.93(\mathrm{~m}, 2 \mathrm{H}), 12.09$ (broad s, disappeared on treatment with $\left.\mathrm{D}_{2} \mathrm{O}, 1 \mathrm{H}\right)$. IR: $v 3318 \mathrm{~cm}^{-1}$. Anal. $\left(\mathrm{C}_{23} \mathrm{H}_{20} \mathrm{FNO}_{3} \mathrm{~S}\right.$ (409.47)) C, H, N, F, S.

2-(2-Methoxyphenyl)-3-((3,4,5-trimethoxyphenyl)thio)-1 H-indole (45)—45 was synthesized similarly to $\mathbf{1 7}$, starting from $\mathbf{8 9}$. Yield $5 \%$, mp $160-165{ }^{\circ} \mathrm{C}$ (from ethanol). ${ }^{1} \mathrm{H}$ $\operatorname{NMR}\left(\mathrm{CDCl}_{3}\right): \delta 3.64(\mathrm{~s}, 6 \mathrm{H}), 3.77(\mathrm{~s}, 3 \mathrm{H}), 3.92(\mathrm{~s}, 3 \mathrm{H}), 6.35(\mathrm{~s}, 2 \mathrm{H}), 7.04-7.08(\mathrm{~m}, 2 \mathrm{H})$, 7.15-7.19 (m, 1H), 7.25-7.29 (m, 1H), 7.36-7.40 (m, 1H), 7.45-7.47 (m, 1H), 7.67-7.69 $(\mathrm{m}, 1 \mathrm{H}), 7.97-7.99(\mathrm{~m}, 1 \mathrm{H}), 9.40 \mathrm{ppm}$ (broad s, disappeared on treatment with $\left.\mathrm{D}_{2} \mathrm{O}, 1 \mathrm{H}\right)$. IR: $v 3338 \mathrm{~cm}^{-1}$. Anal. $\left(\mathrm{C}_{24} \mathrm{H}_{23} \mathrm{NO}_{4} \mathrm{~S}(421.51)\right) \mathrm{C}, \mathrm{H}, \mathrm{N}, \mathrm{S}$.

2-(3-Methoxyphenyl)-3-((3,4,5-trimethoxyphenyl)thio)-1 H-indole (46)—-46 was synthesized similarly to $\mathbf{1 7}$, starting from $\mathbf{9 0}$. Yield $4 \%, \mathrm{mp} 192-194{ }^{\circ} \mathrm{C}$ (from ethanol). ${ }^{1} \mathrm{H}$ $\operatorname{NMR}\left(\mathrm{CDCl}_{3}\right): \delta 3.63(\mathrm{~s}, 6 \mathrm{H}), 3.75(\mathrm{~s}, 3 \mathrm{H}), 3.76(\mathrm{~s}, 3 \mathrm{H}), 6.36(\mathrm{~s}, 2 \mathrm{H}), 6.93-6.96(\mathrm{~m}, 1 \mathrm{H})$, 7.18-7.21 (m, 1H), 7.25-7.40 (m, 4H), 7.44-7.46 (m, 1H), 7.69-7.71 (m, 1H), 8.57 ppm (broad s, disappeared on treatment with $\left.\mathrm{D}_{2} \mathrm{O}, 1 \mathrm{H}\right)$. IR: $v 3333 \mathrm{~cm}^{-1}$. Anal. $\left(\mathrm{C}_{24} \mathrm{H}_{23} \mathrm{NO}_{4} \mathrm{~S}\right.$ (421.51)) C, H, N, S.

2-(4-Methoxyphenyl)-3-((3,4,5-trimethoxyphenyl)thio)-1 H-indole (47)—47 was synthesized similarly to 17 , starting from 91 . Yield $8 \%$, mp $165-170{ }^{\circ} \mathrm{C}$ (from ethanol). ${ }^{1} \mathrm{H}$ NMR $\left(\mathrm{CDCl}_{3}\right): \delta 3.63(\mathrm{~s}, 6 \mathrm{H}), 3.78(\mathrm{~s}, 3 \mathrm{H}), 3.85(\mathrm{~s}, 3 \mathrm{H}), 6.37(\mathrm{~s}, 2 \mathrm{H}), 6.97-6.99(\mathrm{~m}, 2 \mathrm{H})$, 7.17-7.23 (m, 2H), 7.41-7.43 (m, 1H), 7.66-7.74 (m, 3H), $8.68 \mathrm{ppm}$ (broad s, disappeared on treatment with $\left.\mathrm{D}_{2} \mathrm{O}, 1 \mathrm{H}\right)$. IR: $v 3324 \mathrm{~cm}^{-1}$. Anal. $\left(\mathrm{C}_{24} \mathrm{H}_{23} \mathrm{NO}_{4} \mathrm{~S}(421.51)\right) \mathrm{C}, \mathrm{H}, \mathrm{N}, \mathrm{S}$.

2-(Pyridin-2-yl)-3-((3,4,5-trimethoxyphenyl)thio)-1 $\boldsymbol{H}$-indole (53)—53 was synthesized similarly to $\mathbf{1 7}$, starting from $\mathbf{9 2}$. Yield $26 \%$ as an oil. ${ }^{1} \mathrm{H} \mathrm{NMR}\left(\mathrm{CDCl}_{3}\right): \delta 3.63$ (s, 6H), $3.76(\mathrm{~s}, 3 \mathrm{H}), 6.39(\mathrm{~s}, 2 \mathrm{H}), 7.18(\mathrm{t}, J=7.1 \mathrm{~Hz}, 1 \mathrm{H}), 7.24-7.27(\mathrm{~m}, 2 \mathrm{H}), 7.45(\mathrm{~d}, J=$ $8.1 \mathrm{~Hz}, 1 \mathrm{H}), 7.70-7.77(\mathrm{~m}, 2 \mathrm{H}), 8.63-8.64(\mathrm{~m}, 1 \mathrm{H}), 8.73(\mathrm{~d}, J=8.1 \mathrm{~Hz}, 1 \mathrm{H}), 10.23 \mathrm{ppm}$ (broad s, disappeared on treatment with $\left.\mathrm{D}_{2} \mathrm{O}, 1 \mathrm{H}\right)$. IR: $v 2828,2933,3003,3056 \mathrm{~cm}^{-1}$. Anal. $\left(\mathrm{C}_{22} \mathrm{H}_{20} \mathrm{~N}_{2} \mathrm{O}_{3} \mathrm{~S}(392.47)\right) \mathrm{C}, \mathrm{H}, \mathrm{N}, \mathrm{S}$.

2-(Pyridin-3-yl)-3-((3,4,5-trimethoxyphenyl)thio)-1 $\boldsymbol{H}$-indole (55)-55 was synthesized similarly to $\mathbf{1 7}$, starting from $\mathbf{9 3}$. Yield $20 \%$, mp $188-191{ }^{\circ} \mathrm{C}$ (from ethanol). ${ }^{1} \mathrm{H}$ NMR $\left(\mathrm{CDCl}_{3}\right): \delta 3.65(\mathrm{~s}, 6 \mathrm{H}), 3.76(\mathrm{~s}, 3 \mathrm{H}), 6.33(\mathrm{~s}, 2 \mathrm{H}), 7.22(\mathrm{~m}, 1 \mathrm{H}), 7.31(\mathrm{~m}, 1 \mathrm{H}), 7.37-$ $7.41(\mathrm{~m} 1 \mathrm{H}), 7.48(\mathrm{~d}, J=8.1 \mathrm{~Hz}, 1 \mathrm{H}), 7.70(\mathrm{~d}, J=7.6 \mathrm{~Hz}, 1 \mathrm{H}), 8.16-8.17(\mathrm{~m}, 1 \mathrm{H}), 8.62(\mathrm{~m}$, $1 \mathrm{H}), 9.02(\mathrm{~m}, 1 \mathrm{H}), 9.26 \mathrm{ppm}$ (broad s, disappeared on treatment with $\left.\mathrm{D}_{2} \mathrm{O}, 1 \mathrm{H}\right) .{ }^{13} \mathrm{C}$ NMR (DMSO- $d_{6}$ ): $\delta 56.28,60.48,98.8,103.33,112.63,119.31,121.21,123.66,124.12,127.75$, 
130.72, 133.85, 135.99, 136.82, 139.7, 149.19, 149.68, 153.77 ppm. IR: $v 2923,3674 \mathrm{~cm}^{-1}$. Anal. $\left(\mathrm{C}_{22} \mathrm{H}_{20} \mathrm{~N}_{2} \mathrm{O}_{3} \mathrm{~S}(392.47)\right) \mathrm{C}, \mathrm{H}, \mathrm{N}$.

2-(Pyridin-4-yl)-3-((3,4,5-trimethoxyphenyl)thio)-1H-indole (57)-57 was synthesized similarly to $\mathbf{1 7}$, starting from $\mathbf{9 4}$. Yield $60 \%$ as a yellow solid, $\mathrm{mp} 215-218{ }^{\circ} \mathrm{C}$ (from ethanol). ${ }^{1} \mathrm{H}$ NMR (DMSO- $\left.d_{6}\right): \delta 3.53(\mathrm{~s}, 6 \mathrm{H}), 3.57(\mathrm{~s}, 3 \mathrm{H}), 6.32(\mathrm{~s}, 2 \mathrm{H}), 7.14-7.18$ $(\mathrm{m}, 1 \mathrm{H}), 7.27-7.31(\mathrm{~m}, 1 \mathrm{H}), 7.53-7.57(\mathrm{~m}, 2 \mathrm{H}), 7.92(\mathrm{dd}, J=1.7$ and $4.5 \mathrm{~Hz}, 2 \mathrm{H}), 8.71(\mathrm{dd}$, $J=1.6$ and $4.5 \mathrm{~Hz}, 2 \mathrm{H}), 12.36 \mathrm{ppm}$ (broad s, disappeared on treatment with $\mathrm{D}_{2} \mathrm{O}, 1 \mathrm{H}$ ). ${ }^{13} \mathrm{C}$ NMR (DMSO- $\left.d_{6}\right): \delta 56.27,60.47,100.49,103.60,112.82,119.63,121.38,122.5,124.25$, $130.89,133.39,135.99,136.92,138.76,139.02,150.52,153.8 \mathrm{ppm}$. IR: $v 3320 \mathrm{~cm}^{-1}$. Anal. $\left(\mathrm{C}_{22} \mathrm{H}_{20} \mathrm{~N}_{2} \mathrm{O}_{3} \mathrm{~S}(392.47)\right) \mathrm{C}, \mathrm{H}, \mathrm{N}$.

2-Cyclobutyl-3-((3,4,5-trimethoxyphenyl)thio)-1 $\boldsymbol{H}$-indole (73)-73 was synthesized similarly to 17 , starting from 95 . Yield $56 \%, \mathrm{mp} 149-153{ }^{\circ} \mathrm{C}$ (from ethanol). ${ }^{1} \mathrm{H}$ NMR (DMSO- $\left.d_{6}\right): \delta 1.85-1.92(\mathrm{~m}, 1 \mathrm{H}), 1.98-2.10(\mathrm{~m}, 1 \mathrm{H}), 2.23-2.28(\mathrm{~m}, 2 \mathrm{H}), 2.28-2.30(\mathrm{~m}$, $2 \mathrm{H}), 2.57$ (s, 9H), 3.94-4.03 (m, 1H), $6.27(\mathrm{~s}, 2 \mathrm{H}), 7.01-7.05(\mathrm{~m}, 1 \mathrm{H}), 7.11-7.15(\mathrm{~m}, 1 \mathrm{H})$, $7.38(\mathrm{~d}, J=7.8 \mathrm{~Hz}, 1 \mathrm{H}), 7.43(\mathrm{~d}, J=8.0 \mathrm{~Hz}, 1 \mathrm{H}), 11.74 \mathrm{ppm}$ (broad s, disappeared on treatment with $\left.\mathrm{D}_{2} \mathrm{O}, 1 \mathrm{H}\right)$. IR: $v 3361 \mathrm{~cm}^{-1}$. Anal. $\left(\mathrm{C}_{21} \mathrm{H}_{23} \mathrm{NO}_{3} \mathrm{~S}(369.48)\right) \mathrm{C}, \mathrm{H}, \mathrm{N}, \mathrm{S}$.

2-Cyclopentyl-3-((3,4,5-trimethoxyphenyl)thio)-1 $\mathbf{H}$-indole (75)-75 was synthesized similarly to $\mathbf{1 7}$, starting from 96 . Yield $56 \%, \mathrm{mp} 178-183{ }^{\circ} \mathrm{C}$ (from ethanol). ${ }^{1} \mathrm{H}$ NMR (DMSO- $\left.d_{6}\right): \delta 1.66-1.68(\mathrm{~m}, 2 \mathrm{H}), 1.17-1.85(\mathrm{~m}, 4 \mathrm{H}), 1.95-1.98(\mathrm{~m}, 2 \mathrm{H}), 3.52-3.54$ $(\mathrm{m}, 1 \mathrm{H}), 3.56(\mathrm{~s}, 6 \mathrm{H}), 3.57(\mathrm{~s}, 3 \mathrm{H}), 6.28(\mathrm{~s}, 2 \mathrm{H}), 7.0-7.04(\mathrm{~m}, 1 \mathrm{H}), 7.09-7.13(\mathrm{~m}, 1 \mathrm{H}), 7.36-$ $7.41(\mathrm{~m}, 2 \mathrm{H}), 11.51 \mathrm{ppm}$ (broad s, disappeared on treatment with $\left.\mathrm{D}_{2} \mathrm{O}, 1 \mathrm{H}\right)$. IR: $v 3331$ $\mathrm{cm}^{-1}$. Anal. $\left(\mathrm{C}_{22} \mathrm{H}_{25} \mathrm{NO}_{3} \mathrm{~S}(383.50)\right) \mathrm{C}, \mathrm{H}, \mathrm{N}, \mathrm{S}$.

2-Cyclohexyl-3-((3,4,5-trimethoxyphenyl)thio)-1 $\mathbf{H}$-indole (77)—-77 was synthesized similarly to $\mathbf{1 7}$, starting from 97 . Yield $48 \%, \mathrm{mp} 214-219^{\circ} \mathrm{C}$ (from ethanol). ${ }^{1} \mathrm{H}$ NMR (DMSO- $d_{6}$ ): $\delta 1.24-1.42(\mathrm{~m}, 3 \mathrm{H}), 1.66-1.83(\mathrm{~m}, 7 \mathrm{H}), 3.09-3.17(\mathrm{~m}, 1 \mathrm{H}), 3.56(\mathrm{~s}, 6 \mathrm{H}), 3.57$ (s, 3H), $6.28(\mathrm{~s}, 2 \mathrm{H}), 7.0-7.02(\mathrm{~m}, 1 \mathrm{H}), 7.09-7.13(\mathrm{~m}, 1 \mathrm{H}), 7.35-7.41(\mathrm{~m}, 2 \mathrm{H}), 11.52 \mathrm{ppm}$ (broad s, disappeared on treatment with $\left.\mathrm{D}_{2} \mathrm{O}, 1 \mathrm{H}\right)$. IR: $v 3327 \mathrm{~cm}^{-1}$. Anal. $\left(\mathrm{C}_{23} \mathrm{H}_{27} \mathrm{NO}_{3} \mathrm{~S}\right.$ (397.53)) C, H, N, S.

General Procedure for the Preparation of Compounds 14, 16, 37, 48, 59, 62, and 72. Example: 2-(1 H-Pyrazol-4-yl)-3-((3,4,5-trimethoxyphenyl)thio)-1 $\mathrm{H}$-indole (14)

2-(1H-Pyrazol-4-yl)-1 $H$-indole (98) (0.08 g, $0.00044 \mathrm{~mol})$ was added to an ice-cooled suspension of $\mathrm{NaH}(0.058 \mathrm{~g}, 0.00145 \mathrm{~mol}, 60 \%$ dispersion in mineral oil) in anhydrous DMF (3 mL) under an Ar stream. After $15 \mathrm{~min}$, bis(3,4,5-trimethoxyphenyl)disulfide (0.21 g, $0.00053 \mathrm{~mol}$ ) was added, and the reaction mixture was heated at $60^{\circ} \mathrm{C}$ for $12 \mathrm{~h}$ under an Ar stream. After cooling, the reaction mixture was quenched on crushed ice and extracted with ethyl acetate. The organic layer was washed with brine, dried, and filtered. Removal of the solvent gave a residue that was purified by column chromatography (silica gel, ethyl acetate/ $n$-hexane $=7: 3)$ to furnish $14(0.1 \mathrm{~g}, 59 \%), \mathrm{mp} 178-182{ }^{\circ} \mathrm{C}$ (from ethanol). ${ }^{1} \mathrm{H}$ NMR (DMSO- $\left.d_{6}\right): \delta 3.53(\mathrm{~s}, 6 \mathrm{H}), 3.57(\mathrm{~s}, 3 \mathrm{H}), 6.33(\mathrm{~s}, 2 \mathrm{H}), 7.06-7.10(\mathrm{~m}, 1 \mathrm{H}), 7.15-7.19(\mathrm{~m}$, $1 \mathrm{H}), 7.44-7.46(\mathrm{~m}, 2 \mathrm{H}), 8.23(\mathrm{~m}, 2 \mathrm{H}), 11.88$ (broad s, disappeared on treatment with $\mathrm{D}_{2} \mathrm{O}$, $1 \mathrm{H}), 13.17 \mathrm{ppm}$ (broad s, disappeared on treatment with $\mathrm{D}_{2} \mathrm{O}, 1 \mathrm{H}$ ). IR: $v 3158,3291 \mathrm{~cm}^{-1}$. Anal. $\left(\mathrm{C}_{20} \mathrm{H}_{19} \mathrm{~N}_{3} \mathrm{O}_{3} \mathrm{~S}(381.45)\right) \mathrm{C}, \mathrm{H}, \mathrm{N}, \mathrm{S}$.

2-(1 H-Pyrazol-3-yl)-3-((3,4,5-trimethoxyphenyl)thio)-1H-indole (16)-16 was synthesized similarly to $\mathbf{1 4}$, starting from $\mathbf{9 9} .{ }^{22}$ Yield $35 \%$, mp $194-197{ }^{\circ} \mathrm{C}$ (from ethanol). ${ }^{1} \mathrm{H}$ NMR (DMSO- $\left.d_{6}\right): \delta 3.51(\mathrm{~s}, 6 \mathrm{H}), 3.55(\mathrm{~s}, 3 \mathrm{H}), 6.35(\mathrm{~s}, 2 \mathrm{H}), 6.97-6.98(\mathrm{~m}$, $1 \mathrm{H}), 7.07$ (t, $J=7.6 \mathrm{~Hz}, 1 \mathrm{H}), 7.17$ (t, $J=7.9 \mathrm{~Hz}, 1 \mathrm{H}), 7.46-7.49(\mathrm{~m}, 2 \mathrm{H}), 7.83(\mathrm{~s}, 1 \mathrm{H}), 11.97$ 
(broad s, disappeared on treatment with $\mathrm{D}_{2} \mathrm{O}, 1 \mathrm{H}$ ), $13.23 \mathrm{ppm}$ (broad s, disappeared on treatment with $\left.\mathrm{D}_{2} \mathrm{O}, 1 \mathrm{H}\right)$. IR: $v 2936,3348 \mathrm{~cm}^{-1}$. Anal. $\left(\mathrm{C}_{20} \mathrm{H}_{19} \mathrm{~N}_{3} \mathrm{O}_{3} \mathrm{~S}(381.45)\right) \mathrm{C}, \mathrm{H}, \mathrm{N}$, S.

2-(p-Tolyl)-3-((3,4,5-trimethoxyphenyl)thio)-1 $\boldsymbol{H}$-indole (37)-37 was synthesized similarly to 14, starting from 100. Yield $72 \%$, mp $168-171{ }^{\circ} \mathrm{C}$ (from ethanol). ${ }^{1} \mathrm{H}$ NMR $\left(\right.$ DMSO- $\left.d_{6}\right): \delta 2.36(\mathrm{~s}, 3 \mathrm{H}), 3.52(\mathrm{~s}, 6 \mathrm{H}), 3.56(\mathrm{~s}, 3 \mathrm{H}), 6.29(\mathrm{~s}, 2 \mathrm{H}), 7.09-7.13(\mathrm{~m}, 1 \mathrm{H})$, 7.19-7.23 (m, 1H), 7.32-7.34 (m, 2H), 7.47-7.51 (m, 2H), 7.78-7.80 (m, 2H), $12.02 \mathrm{ppm}$ (broad s, disappeared on treatment with $\left.\mathrm{D}_{2} \mathrm{O}, 1 \mathrm{H}\right)$. IR: $v 3344 \mathrm{~cm}^{-1}$. Anal. $\left(\mathrm{C}_{24} \mathrm{H}_{23} \mathrm{NO}_{3} \mathrm{~S}\right.$ (405.51)) C, H, N, S.

\section{2-(3-Isopropoxy-4-methoxyphenyl)-3-((3,4,5-trimethoxyphenyl)-thio)-1 $\mathrm{H}$-indole} (48)-48 was synthesized similarly to $\mathbf{1 4}$, starting from $\mathbf{1 0 1}$. Yield $53 \%, \mathrm{mp} 110-113{ }^{\circ} \mathrm{C}$ (from ethanol). ${ }^{1} \mathrm{H}$ NMR $\left(\mathrm{CDCl}_{3}\right): \delta 1.29(\mathrm{~d}, J=6.1 \mathrm{~Hz}, 6 \mathrm{H}), 3.65(\mathrm{~s}, 6 \mathrm{H}), 3.77(\mathrm{~s}, 3 \mathrm{H})$, $3.90(\mathrm{~s}, 3 \mathrm{H}), 4.35(\mathrm{~m}, 1 \mathrm{H}), 6.36(\mathrm{~s}, 2 \mathrm{H}), 6.97$ (d, $J=8.4 \mathrm{~Hz}, 1 \mathrm{H}), 7.19-7.29(\mathrm{~m}, 3 \mathrm{H}), 7.41$ (m, 1H), 7.44-7.46 (m, 1H), 7.70-7.72 (m, 1H), 8.56 ppm (broad s, disappeared on treatment with $\left.\mathrm{D}_{2} \mathrm{O}, 1 \mathrm{H}\right)$. IR: $v 3337 \mathrm{~cm}^{-1}$. Anal. $\left(\mathrm{C}_{27} \mathrm{H}_{29} \mathrm{NO}_{5} \mathrm{~S}(479.59)\right) \mathrm{C}, \mathrm{H}, \mathrm{N}, \mathrm{S}$.

2-(Naphthalen-1-yl)-3-((3,4,5-trimethoxyphenyl)thio)-1 $\boldsymbol{H}$-indole (59)-59 was synthesized similarly to $\mathbf{1 4}$, starting from $\mathbf{1 0 2}$. Yield $44 \%, \mathrm{mp} 227-230{ }^{\circ} \mathrm{C}$ (from ethanol). ${ }^{1} \mathrm{H} \mathrm{NMR}\left(\mathrm{CDCl}_{3}\right): \delta 3.56(\mathrm{~s}, 6 \mathrm{H}), 3.71(\mathrm{~s}, 3 \mathrm{H}), 6.30(\mathrm{~s}, 2 \mathrm{H}), 7.22-7.26(\mathrm{~m}, 1 \mathrm{H})$, 7.29-7.33 (m, 1H), 7.39-7.55 (m, 4H), $7.61(\mathrm{dd}, J=1.2$ and $7.1 \mathrm{~Hz}, 1 \mathrm{H}), 7.74-7.76(\mathrm{~m}, 1 \mathrm{H})$, 7.82-7.84 (m, 1H), 7.90-7.95 (m, 2H), $8.59 \mathrm{ppm}$ (broad s, disappeared on treatment with $\left.\mathrm{D}_{2} \mathrm{O}, 1 \mathrm{H}\right)$. IR: $v 3229 \mathrm{~cm}^{-1}$. Anal. $\left(\mathrm{C}_{27} \mathrm{H}_{23} \mathrm{NO}_{3} \mathrm{~S}(441.54)\right) \mathrm{C}, \mathrm{H}, \mathrm{N}, \mathrm{S}$.

2-(Naphthalen-2-yl)-3-((3,4,5-trimethoxyphenyl)thio)-1 $H$-indole (62)—62 was synthesized similarly to $\mathbf{1 4}$, starting from $\mathbf{1 0 3}$. Yield $44 \%$, mp $157-160{ }^{\circ} \mathrm{C}$ (from ethanol). ${ }^{1} \mathrm{H} \mathrm{NMR}\left(\mathrm{CDCl}_{3}\right): \delta 3.65(\mathrm{~s}, 6 \mathrm{H}), 3.79(\mathrm{~s}, 3 \mathrm{H}), 6.42(\mathrm{~s}, 2 \mathrm{H}), 7.22-7.34(\mathrm{~m}, 2 \mathrm{H})$, 7.50-7.56 (m, 3H), 7.74-7.75 (m, 1H), 7.88-7.90 (m, 2H), 7.93-8.00 (m, 2H), 8.25 (m, 1H), $8.71 \mathrm{ppm}$ (broad s, disappeared on treatment with $\mathrm{D}_{2} \mathrm{O}, 1 \mathrm{H}$ ). IR: $v 3318 \mathrm{~cm}^{-1}$. Anal. $\left(\mathrm{C}_{27} \mathrm{H}_{23} \mathrm{NO}_{3} \mathrm{~S}(441.54)\right) \mathrm{C}, \mathrm{H}, \mathrm{N}, \mathrm{S}$.

2-((1,1'-Biphenyl)-4-yl)-3-((3,4,5-trimethoxyphenyl)thio)-1 H-indole (72)-72 was synthesized similarly to $\mathbf{1 4}$, starting from 104 . Yield $52 \%, \mathrm{mp} 210-215^{\circ} \mathrm{C}$ (from ethanol). ${ }^{1} \mathrm{H}$ NMR (DMSO- $\left.d_{6}\right): \delta 3.54(\mathrm{~s}, 6 \mathrm{H}), 3.57(\mathrm{~s}, 3 \mathrm{H}), 6.33(\mathrm{~s}, 2 \mathrm{H}), 7.11-7.15(\mathrm{~m}$, $1 \mathrm{H}), 7.23-7.25(\mathrm{~m}, 1 \mathrm{H}), 7.38-7.42(\mathrm{~m}, 1 \mathrm{H}), 7.48-7.54(\mathrm{~m}, 4 \mathrm{H}), 7.75-7.77(\mathrm{~m}, 2 \mathrm{H}), 7.84-$ $7.86(\mathrm{~m}, 2 \mathrm{H}), 8.01-8.03(\mathrm{~m}, 2 \mathrm{H}), 12.14 \mathrm{ppm}$ (broad s, disappeared on treatment with $\mathrm{D}_{2} \mathrm{O}$, 1H). IR: $v 3329 \mathrm{~cm}^{-1}$. Anal. $\left(\mathrm{C}_{29} \mathrm{H}_{25} \mathrm{NO}_{3} \mathrm{~S}(467.58)\right) \mathrm{C}, \mathrm{H}, \mathrm{N}, \mathrm{S}$.

2-(3-((3,4,5-Trimethoxyphenyl)thio)-1 $\mathrm{H}$-indol-2-yl)-4,5-dihy-droxazole (28)-2Bromoethylamine hydrobromide $(0.63 \mathrm{~g}, 0.0031 \mathrm{~mol})$ was added to a solution of 3-((3,4,5trimethoxyphenyl)thio)-1 $H$-indole-2-carboxylic acid (107) $(1.0 \mathrm{~g}, 0.0028 \mathrm{~mol})$, triethylamine $(0.85 \mathrm{~g}, 1.2 \mathrm{~mL}, 0.0084 \mathrm{~mol})$, and BOP reagent $(1.24 \mathrm{~g}, 0.0028 \mathrm{~mol})$ in anhydrous DMF $(15 \mathrm{~mL})$. The reaction mixture was stirred at $25^{\circ} \mathrm{C}$ for $2 \mathrm{~h}$ and then diluted with water and extracted with ethyl acetate. The organic layer was washed with brine, dried, and filtered. Removal of the solvent gave a residue that was purified by column chromatography (silica gel, ethyl acetate $/ n$-hexane $=7: 3$ as eluent) to furnish $28(0.31 \mathrm{~g}$, $30 \%$ ), mp 190-195 ${ }^{\circ} \mathrm{C}$ (from ethanol). ${ }^{1} \mathrm{H}$ NMR (DMSO- $\left.d_{6}\right): \delta 3.55(\mathrm{~s}, 6 \mathrm{H}), 3.56(\mathrm{~s}, 3 \mathrm{H})$, $4.00(\mathrm{t}, J=9.7 \mathrm{~Hz}, 2 \mathrm{H}), 4.43(\mathrm{t}, J=9.5 \mathrm{~Hz}, 2 \mathrm{H}), 6.42(\mathrm{~s}, 2 \mathrm{H}), 7.07-7.11(\mathrm{~m}, 1 \mathrm{H}), 7.23-7.27$ $(\mathrm{m}, 1 \mathrm{H}), 7.43-7.49(\mathrm{~m}, 2 \mathrm{H}), 12.21 \mathrm{ppm}$ (broad s, disappeared on treatment with $\left.\mathrm{D}_{2} \mathrm{O}, 1 \mathrm{H}\right)$. IR: $v 3198 \mathrm{~cm}^{-1}$. Anal. $\left(\mathrm{C}_{20} \mathrm{H}_{20} \mathrm{~N}_{2} \mathrm{O}_{4} \mathrm{~S}(384.45)\right) \mathrm{C}, \mathrm{H}, \mathrm{N}, \mathrm{S}$. 
2-(3-((3,4,5-Trimethoxyphenyl)thio)-1 $H$-indol-2-yl)oxazole (31)-A mixture of 2iodo-3-((3,4,5-trimethoxyphenyl)thio)-1 H-indole (108) (0.1 g, $0.00023 \mathrm{~mol}), 2$ (tributylstannyl)oxazole (0.25 g, $0.1 \mathrm{~mL}, 0.00069 \mathrm{~mol}), \mathrm{P}(\mathrm{Cy})_{3}(0.0064 \mathrm{~g}, 0.000023 \mathrm{~mol})$ in anhydrous DMF $(2 \mathrm{~mL})$ was degassed for $10 \mathrm{~min}$. $\operatorname{Pd}_{2}(\mathrm{dba})_{3}(0.01 \mathrm{~g}, 0.000011 \mathrm{~mol})$ was added, and the reaction mixture was placed into the microwave cavity (closed vessel mode, $P_{\max }=250 \mathrm{psi}$ ). A starting microwave irradiation of $150 \mathrm{~W}$ was used, the temperature being ramped from 25 to $150{ }^{\circ} \mathrm{C}$ while stirring. Once $150{ }^{\circ} \mathrm{C}$ was reached, taking about $2 \mathrm{~min}$, the reaction mixture was held at this temperature for $10 \mathrm{~min}$. The reaction was quenched on a mixture of a saturated aqueous solution of potassium carbonate $(20 \mathrm{~mL})$ and ethyl acetate while stirring for $30 \mathrm{~min}$. The organic layer was separated and washed with brine, dried, and filtered. Removal of the solvent gave a residue that was purified by column chromatography (silica gel, ethyl acetate $/ n$-hexane $=1: 3$ as eluent) to furnish $\mathbf{3 1}(0.03,35 \%), \mathrm{mp} 80-83{ }^{\circ} \mathrm{C}$ (from ethanol). ${ }^{1} \mathrm{H}$ NMR (DMSO- $\left.d_{6}\right): \delta 3.56(\mathrm{~s}, 6 \mathrm{H}), 3.57(\mathrm{~s}, 3 \mathrm{H}), 6.38(\mathrm{~s}, 2 \mathrm{H}), 7.06-7.10$ $(\mathrm{m}, 1 \mathrm{H}), 7.16-7.20(\mathrm{~m}, 1 \mathrm{H}), 7.40-7.52(\mathrm{~m}, 3 \mathrm{H}), 7.76-7.77(\mathrm{~m}, 1 \mathrm{H}), 11.66 \mathrm{ppm}$ (broad s, disappeared on treatment with $\left.\mathrm{D}_{2} \mathrm{O}, 1 \mathrm{H}\right)$. IR: $v 3340 \mathrm{~cm}^{-1}$. Anal. $\left(\mathrm{C}_{20} \mathrm{H}_{18} \mathrm{~N}_{2} \mathrm{O}_{4} \mathrm{~S}(382.43)\right)$ $\mathrm{C}, \mathrm{H}, \mathrm{N}, \mathrm{S}$.

6-(3-((3,4,5-Trimethoxyphenyl)thio)-1 H-indol-2-yl)quinoline (66)—A mixture of $108(0.1 \mathrm{~g}, 0.00023 \mathrm{~mol})$, 6-quinolineboronic acid pinacol ester (0.076 g, $0.0003 \mathrm{~mol})$, and potassium phosphate $(0.14 \mathrm{~g}, 0.00068 \mathrm{~mol})$ in anhydrous DMF $(10 \mathrm{~mL})$ was degassed for 30 min. $\mathrm{Pd}$ (dppf) $\mathrm{Cl}_{2} \cdot \mathrm{CH}_{2} \mathrm{Cl}_{2}(0.0054 \mathrm{~g}, 0.0000066 \mathrm{~mol}$, complex with dichloromethane (1:1), $\mathrm{Pd} 13 \%$ ) was added under an Ar stream, and the reaction mixture was heated at 110 ${ }^{\circ} \mathrm{C}$ for $12 \mathrm{~h}$. After cooling, the reaction mixture was diluted with water and extracted with ethyl acetate. The organic layer was washed with brine, dried, and filtered. Removal of the solvent gave a residue that was purified by column chromatography (silica gel, ethyl acetate/ $n$-hexane $=8: 2$ as eluent) to furnish 66 as a slurry $(0.07 \mathrm{~g}, 70 \%) .{ }^{1} \mathrm{H}$ NMR (DMSO- $\left.d_{6}\right): \delta$ $3.52(\mathrm{~s}, 6 \mathrm{H}), 3.55$ (s, 3H), $6.35(\mathrm{~s}, 2 \mathrm{H}), 7.14-7.16(\mathrm{~m}, 1 \mathrm{H}), 7.25-7.28(\mathrm{~m}, 1 \mathrm{H}), 7.54-7.61$ $(\mathrm{m}, 3 \mathrm{H}), 8.15(\mathrm{~d}, J=8.8 \mathrm{~Hz}, 1 \mathrm{H}), 8.32(\mathrm{dd}, J=2.0$ and $8.8 \mathrm{~Hz}, 1 \mathrm{H}), 8.42(\mathrm{~d}, J=7.3 \mathrm{~Hz}, 1 \mathrm{H})$, $8.47(\mathrm{~d}, J=1.8 \mathrm{~Hz}, 1 \mathrm{H}), 8.95(\mathrm{dd}, J=1.7$ and $4.2 \mathrm{~Hz}, 1 \mathrm{H}), 12.29 \mathrm{ppm}$ (broad s, disappeared on treatment with $\left.\mathrm{D}_{2} \mathrm{O}, 1 \mathrm{H}\right)$. IR: $v 2922,3305 \mathrm{~cm}^{-1}$. Anal. $\left(\mathrm{C}_{26} \mathrm{H}_{22} \mathrm{~N}_{2} \mathrm{O}_{3} \mathrm{~S}(442.53)\right) \mathrm{C}, \mathrm{H}$, $\mathrm{N}, \mathrm{S}$.

3-((3,4,5-Trimethoxyphenyl)thio)-1H,1'H-2,5'-bisindole (67)-67 was synthesized similarly to 66, starting from $\mathbf{1 0 8}$ and 1-Boc-indole-5-boronic acid pinacol ester. Yield $26 \%$ as a slurry. ${ }^{1} \mathrm{H}$ NMR $\left(\mathrm{CDCl}_{3}\right): \delta 3.64(\mathrm{~s}, 6 \mathrm{H}), 3.79(\mathrm{~s}, 3 \mathrm{H}), 6.41(\mathrm{~s}, 2 \mathrm{H}), 6.61-6.62(\mathrm{~m}, 1 \mathrm{H})$, 7.21-7.28 (m, 3H), 7.45-7.47 (m, 2H), 7.64-7.71 (m, 2H), 8.05-8.06 (m, 1H), 8.32 (broad s, disappeared on treatment with $\left.\mathrm{D}_{2} \mathrm{O}, 1 \mathrm{H}\right), 8.65 \mathrm{ppm}$ (broad s, disappeared on treatment with $\left.\mathrm{D}_{2} \mathrm{O}, 1 \mathrm{H}\right)$. IR: $v 2963,2918,3350 \mathrm{~cm}^{-1}$. Anal. $\left(\mathrm{C}_{25} \mathrm{H}_{22} \mathrm{~N}_{2} \mathrm{O}_{3} \mathrm{~S}(430.52)\right) \mathrm{C}, \mathrm{H}, \mathrm{N}, \mathrm{S}$.

3-((3,4,5-Trimethoxyphenyl)thio)-1 H,1'H-2,2'-bisindole (68)—A mixture of 108 (0.15 g, $0.00034 \mathrm{~mol}), 2$-(triethylstannyl)-1 $H$-indole-1-carboxylic acid ${ }^{24}(0.25 \mathrm{~g}, 0.000683$ $\mathrm{mol})$, and ethanol $(5 \mathrm{~mL})$ was degassed for $30 \mathrm{~min}$. $\mathrm{PdCl}_{2}\left(\mathrm{PPh}_{3}\right)_{2}(0.021 \mathrm{~g}, 0.000031 \mathrm{~mol})$ was added under an Ar stream, and the reaction mixture was heated at $78^{\circ} \mathrm{C}$ for $48 \mathrm{~h}$. After cooling, the mixture was filtered through a pad of Celite, then diluted with water and extracted with ethyl acetate. The organic layer was washed with brine, dried, and filtered. Removal of the solvent gave a residue that was purified by column chromatography (silica gel, ethyl acetate $/ n$-hexane $=1: 2$ as eluent $)$ to furnish $\mathbf{6 8}(0.03 \mathrm{~g}, 20 \%), \mathrm{mp} 220-225^{\circ} \mathrm{C}$ (from ethanol). ${ }^{1} \mathrm{H}$ NMR (DMSO- $\left.d_{6}\right): \delta 3.52(\mathrm{~s}, 6 \mathrm{H}), 3.55(\mathrm{~s}, 3 \mathrm{H}), 6.39(\mathrm{~s}, 2 \mathrm{H}), 7.02-7.06$ (m, 1H), 7.13-7.18 (m, 3H), 7.22-7.26 (m, 1H), 7.49-7.55 (m, 3H), 7.59-7.61 (m, 1H), 11.37 (broad s, disappeared on treatment with $\left.\mathrm{D}_{2} \mathrm{O}, 1 \mathrm{H}\right), 12.02 \mathrm{ppm}$ (broad s, disappeared on treatment with $\left.\mathrm{D}_{2} \mathrm{O}, 1 \mathrm{H}\right)$. IR: $v 3326,3388 \mathrm{~cm}^{-1}$. Anal. $\left(\mathrm{C}_{25} \mathrm{H}_{22} \mathrm{~N}_{2} \mathrm{O}_{3} \mathrm{~S}(430.52)\right) \mathrm{C}, \mathrm{H}$, N, S. 
2-(Benzofuran-2-yl)-3-((3,4,5-trimethoxyphenyl)thio)-1 H-indole (69)-A mixture of $108(0.1 \mathrm{~g}, 0.00023 \mathrm{~mol}), 2$-benzofuranylboronic acid $(0.29 \mathrm{~g}, 0.0018 \mathrm{~mol})$, and potassium carbonate $(0.28 \mathrm{~g}, 0.02 \mathrm{~mol})$ in 1,4-dioxane $(12 \mathrm{~mL})$ containing water $(2 \mathrm{~mL})$ was degassed for $30 \mathrm{~min}$. $\mathrm{Pd}(\mathrm{OCOMe})_{2}(0.052 \mathrm{~g}, 0.00234 \mathrm{~mol})$ was added under an Ar stream, and the reaction mixture was heated at $100{ }^{\circ} \mathrm{C}$ for $24 \mathrm{~h}$. After cooling, the mixture was diluted with water and extracted with ethyl acetate. The organic layer was washed with brine, dried, and filtered. Removal of the solvent gave a residue that was purified by column chromatography (silica gel, ethyl acetate $/ n$-hexane $=1: 2$ as eluent) to furnish $69(0.11 \mathrm{~g}$, $88 \%$ ), mp $190-195{ }^{\circ} \mathrm{C}$ (from ethanol). ${ }^{1} \mathrm{H}$ NMR (DMSO- $d_{6}$ ): $\delta 3.54(\mathrm{~s}, 6 \mathrm{H}), 3.56(\mathrm{~s}, 3 \mathrm{H}$ ), $6.42(\mathrm{~s}, 2 \mathrm{H}), 7.14-7.18(\mathrm{~m}, 1 \mathrm{H}), 7.27-7.33(\mathrm{~m}, 2 \mathrm{H}), 7.37-7.42(\mathrm{~m}, 1 \mathrm{H}), 7.54-7.57(\mathrm{~m}, 2 \mathrm{H})$, 7.64-7.67 $(\mathrm{m}, 2 \mathrm{H}), 7.75-7.76(\mathrm{~m}, 1 \mathrm{H}), 12.49 \mathrm{ppm}$ (broad s, disappeared on treatment with $\left.\mathrm{D}_{2} \mathrm{O}, 1 \mathrm{H}\right)$. IR: $v 3338 \mathrm{~cm}^{-1}$. Anal. $\left(\mathrm{C}_{25} \mathrm{H}_{21} \mathrm{NO}_{4} \mathrm{~S}(431.50)\right) \mathrm{C}, \mathrm{H}, \mathrm{N}, \mathrm{S}$.

\section{2-(Benzo[b]thiophen-2-yl)-3-((3,4,5-trimethoxyphenyl)thio)-1 H-indole (70)-A} mixture of $108(0.1 \mathrm{~g}, 0.00023 \mathrm{~mol})$, benzo[b]thien-2-ylboronic acid (0.04 g, 0.000225 $\mathrm{mol})$, and $1 \mathrm{M}$ potassium carbonate $(0.6 \mathrm{~mL})$ in THF $(5 \mathrm{~mL})$ was degassed for $30 \mathrm{~min}$. $\mathrm{Pd}\left(\mathrm{PPh}_{3}\right)_{4}(0.01 \mathrm{~g}, 0.009 \mathrm{mmol})$ was added under an Ar stream, and the reaction mixture was heated at $60{ }^{\circ} \mathrm{C}$ for $24 \mathrm{~h}$. After cooling, the mixture was diluted with water and extracted with ethyl acetate. The organic layer was washed with brine, dried, and filtered. Removal of the solvent gave a residue that was purified by column chromatography (silica gel, dichloromethane/ethyl acetate $=99: 1$ as eluent $)$ to furnish $70(0.01 \mathrm{~g}, 10 \%)$, mp 220$225^{\circ} \mathrm{C}$ (from ethanol). ${ }^{1} \mathrm{H}$ NMR $\left(\mathrm{CDCl}_{3}\right): \delta 3.66(\mathrm{~s}, 6 \mathrm{H}), 3.78(\mathrm{~s}, 3 \mathrm{H}), 6.44(\mathrm{~s}, 2 \mathrm{H}), 7.21-$ $7.23(\mathrm{~m}, 1 \mathrm{H}), 7.28-7.39(\mathrm{~m}, 3 \mathrm{H}), 7.46(\mathrm{~d}, J=7.6 \mathrm{~Hz}, 1 \mathrm{H}), 7.70(\mathrm{~d}, J=7.6 \mathrm{~Hz}, 1 \mathrm{H}), 7.80$ $7.86(\mathrm{~m}, 3 \mathrm{H}), 8.83 \mathrm{ppm}$ (broad s, disappeared on treatment with $\left.\mathrm{D}_{2} \mathrm{O}, 1 \mathrm{H}\right)$. IR: $v 3303 \mathrm{~cm}^{-1}$. Anal. $\left(\mathrm{C}_{25} \mathrm{H}_{21} \mathrm{NO}_{3} \mathrm{~S}_{2}(447.57)\right) \mathrm{C}, \mathrm{H}, \mathrm{N}, \mathrm{S}$.

\section{2-(Benzo[b]thiophen-3-yl)-3-((3,4,5-trimethoxyphenyl)thio)-1 H-indole (71)-A} mixture of 108 (0.1 g, $0.00023 \mathrm{~mol})$, SPhos (0.0075 g, $0.0182 \mathrm{mmol}), \mathrm{Pd}_{2}(\mathrm{dba})_{3}(0.0042 \mathrm{~g}$, $0.00454 \mathrm{mmol})$, and potassium carbonate $(0.0964 \mathrm{~g}, 0.000454 \mathrm{~mol})$ was degassed for 30 min. A solution of benzo[ $b$ ]thien-3-ylboronic acid $(0.061 \mathrm{~g}, 0.00034 \mathrm{~mol})$ in $n$ - $\mathrm{BuOH}(2$ $\mathrm{mL}$ ) was added under an $\mathrm{Ar}$ stream, and the reaction mixture was heated at $100{ }^{\circ} \mathrm{C}$ for $12 \mathrm{~h}$. After cooling, the mixture was diluted with water, made acidic with $1 \mathrm{M} \mathrm{HCl}(\mathrm{pH} \approx 3-4)$, and extracted with ethyl acetate. The organic layer was washed with brine, dried, and filtered. Removal of the solvent gave a residue that was purified by column chromatography (silica gel, ethyl acetate $/ n$-hexane $=1: 2$ as eluent) to furnish $71(0.037 \mathrm{~g}, 36 \%)$, mp 170-174 ${ }^{\circ} \mathrm{C}$ (from ethanol). ${ }^{1} \mathrm{H}$ NMR (DMSO- $\left.d_{6}\right): \delta 3.48$ (s, 6H), $3.53(\mathrm{~s}, 3 \mathrm{H}), 6.25(\mathrm{~s}, 2 \mathrm{H}), 7.16-$ $7.18(\mathrm{~m}, 1 \mathrm{H}), 7.24-7.28(\mathrm{~m}, 1 \mathrm{H}), 7.45-7.47(\mathrm{~m}, 2 \mathrm{H}), 7.53-7.56(\mathrm{~m}, 2 \mathrm{H}), 7.87-7.89(\mathrm{~m}, 1 \mathrm{H})$, $8.18(\mathrm{~s}, 1 \mathrm{H}), 8.09-8.12(\mathrm{~m}, 1 \mathrm{H}), 12.17 \mathrm{ppm}$ (broad s, disappeared on treatment with $\mathrm{D}_{2} \mathrm{O}$, 1H). IR: $v 3237 \mathrm{~cm}^{-1}$. Anal. $\left(\mathrm{C}_{25} \mathrm{H}_{21} \mathrm{NO}_{3} \mathrm{~S}_{2}(447.57)\right) \mathrm{C}, \mathrm{H}, \mathrm{N}, \mathrm{S}$.

\section{2-Methoxy-5-(3-((3,4,5-trimethoxyphenyl)thio)-1 H-indol-2-yl)phenol (49)-}

Anhydrous aluminum chloride $(0.14 \mathrm{~g}, 0.001 \mathrm{~mol})$ was added to a solution of $\mathbf{4 8}(0.17 \mathrm{~g}$, $0.00035 \mathrm{~mol})$ in anhydrous dichloromethane $(10 \mathrm{~mL})$. The reaction mixture was stirred at $25^{\circ} \mathrm{C}$ for $1.5 \mathrm{~h}$. After dilution with a saturated aqueous solution of ammonium chloride, the mixture was extracted with chloroform. The organic layer was washed with brine, dried, and filtered. Removal of the solvent gave a residue that was purified by column chromatography (silica gel, ethyl acetate $/ n$-hexane $=1: 1$ as eluent) to furnish $\mathbf{4 9}(0.05 \mathrm{~g}, 35 \%), \mathrm{mp} 110-113$ ${ }^{\circ} \mathrm{C}$ (from ethanol). ${ }^{1} \mathrm{H}$ NMR $\left(\mathrm{CDCl}_{3}\right): \delta 3.65(\mathrm{~s}, 6 \mathrm{H}), 3.77(\mathrm{~s}, 3 \mathrm{H}), 3.95(\mathrm{~s}, 3 \mathrm{H}), 5.69(\mathrm{~s}, 1 \mathrm{H})$, $6.36(\mathrm{~s}, 2 \mathrm{H}), 6.94(\mathrm{~d}, J=8.3 \mathrm{~Hz}, 1 \mathrm{H}), 7.16-7.20(\mathrm{~m}, 1 \mathrm{H}), 7.24-7.28(\mathrm{~m}, 1 \mathrm{H}), 7.34(\mathrm{dd}, J=$ 2.2 and $8.3 \mathrm{~Hz}, 1 \mathrm{H}), 7.38(\mathrm{~d}, J=2.2 \mathrm{~Hz}, 1 \mathrm{H}), 7.42-7.44(\mathrm{~m}, 1 \mathrm{H}), 7.66-7.68(\mathrm{~m}, 1 \mathrm{H}), 8.49$ ppm (broad s, disappeared on treatment with $\mathrm{D}_{2} \mathrm{O}, 1 \mathrm{H}$ ). IR: $v 2933,3329 \mathrm{~cm}^{-1}$. Anal. $\left(\mathrm{C}_{24} \mathrm{H}_{23} \mathrm{NO}_{5} \mathrm{~S}(437.51)\right) \mathrm{C}, \mathrm{H}, \mathrm{N}, \mathrm{S}$. 
2-(3,4-Dimethoxyphenyl)-3-((3,4,5-trimethoxyphenyl)thio)-1 H-indole (50)Iodomethane $(0.033 \mathrm{~g}, 0.01 \mathrm{~mL}, 0.00023 \mathrm{~mol})$ was added to a mixture of $49(0.1 \mathrm{~g}, 0.000$ $23 \mathrm{~mol})$ and anhydrous potassium carbonate $(0.032 \mathrm{~g}, 0.00023 \mathrm{~mol})$ in anhydrous DMF (5 $\mathrm{mL}$ ). The reaction mixture was stirred at $25{ }^{\circ} \mathrm{C}$ for $2 \mathrm{~h}$, then diluted with water and extracted with ethyl acetate. The organic layer was washed with brine, dried, and filtered. Removal of the solvent gave a residue that was purified by column chromatography (silica gel, ethyl acetate $/ n$-hexane $=1: 1$ as eluent) to furnish $\mathbf{5 0}(0.02 \mathrm{~g}, 20 \%), \mathrm{mp} 117-118{ }^{\circ} \mathrm{C}$ (from ethanol). ${ }^{1} \mathrm{H} \mathrm{NMR}\left(\mathrm{CDCl}_{3}\right): \delta 3.65(\mathrm{~s}, 6 \mathrm{H}), 3.77(\mathrm{~s}, 3 \mathrm{H}), 3.78(\mathrm{~s}, 3 \mathrm{H}), 3.94(\mathrm{~s}, 3 \mathrm{H}), 6.38(\mathrm{~s}$, $2 \mathrm{H}), 6.97(\mathrm{~d}, J=8.3 \mathrm{~Hz}, 1 \mathrm{H}), 7.19-7.23(\mathrm{~m}, 1 \mathrm{H}), 7.26-7.31(\mathrm{~m}, 2 \mathrm{H}), 7.40(\mathrm{~d}, J=2.1 \mathrm{~Hz}$, 1H), 7.45-7.47 (m, 1H), 7.70-7.72 (m, 1H), $8.57 \mathrm{ppm}$ (broad s, disappeared on treatment with $\left.\mathrm{D}_{2} \mathrm{O}, 1 \mathrm{H}\right)$. IR: $v 3344 \mathrm{~cm}^{-1}$. Anal. $\left(\mathrm{C}_{25} \mathrm{H}_{25} \mathrm{NO}_{5} \mathrm{~S}(451.53)\right) \mathrm{C}, \mathrm{H}, \mathrm{N}, \mathrm{S}$.

Aroyl-1H-indoles. General Procedure for the Preparation of Compounds 7, 15, 19, 54, 56, 58, and 60. Example: (2-(1H-Pyrrol-2-yl)-1H-indol-3-yl)(3,4,5-trimethoxyphenyl)methanone (7)

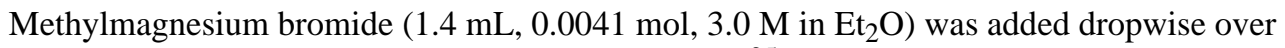
5 min into a mixture of 2-( $1 H$-pyrrol-2-yl)- $1 H$-indole ${ }^{25}(\mathbf{1 0 9})(0.5 \mathrm{~g}, 0.003 \mathrm{~mol})$ and anhydrous $\mathrm{ZnCl}_{2}(0.82 \mathrm{~g}, 0.006 \mathrm{~mol})$ in anhydrous dichloromethane $(20 \mathrm{~mL})$ at $25^{\circ} \mathrm{C}$ under an Ar stream. After $1 \mathrm{~h}$, a solution of 3,4,5-trimethoxybenzoyl chloride $(0.76 \mathrm{~g}, 0.0033 \mathrm{~mol})$ in the same solvent $(16 \mathrm{~mL})$ was added dropwise over 5 min at $25^{\circ} \mathrm{C}$ under an $\mathrm{Ar}$ stream. After an additional $1 \mathrm{~h}, \mathrm{SnCl}_{4}(3 \mathrm{~mL}, 0.003 \mathrm{~mol}, 1.0 \mathrm{M}$ in dichloromethane) was added dropwise, and the reaction mixture was stirred at $25^{\circ} \mathrm{C}$ for $12 \mathrm{~h}$ under an $\mathrm{Ar}$ stream. The reaction was quenched on crushed ice and extracted with dichloro-methane The organic layer was washed with brine, dried, and filtered. Removal of the solvent gave a residue that was purified by column chromatography (silica gel, ethyl acetate $/ n$-hexane $=1: 1$ as eluent) to furnish 7 (0.03 g, 3\%), mp 59-62 ${ }^{\circ} \mathrm{C}$ (from toluene). ${ }^{1} \mathrm{H}$ NMR (DMSO- $\left.d_{6}\right): \delta 3.70$ (s, $6 \mathrm{H}), 3.75(\mathrm{~s}, 3 \mathrm{H}), 6.24-6.26(\mathrm{~m}, 1 \mathrm{H}), 6.84-6.85(\mathrm{~m}, 1 \mathrm{H}), 6.96-7.07(\mathrm{~m}, 5 \mathrm{H}), 7.14-7.18(\mathrm{~m}$, $1 \mathrm{H}), 7.43-7.45(\mathrm{~m}, 1 \mathrm{H}), 11.94$ (broad s, disappeared on treatment with $\left.\mathrm{D}_{2} \mathrm{O}, 1 \mathrm{H}\right), 12.15$ ppm (broad s, disappeared on treatment with $\mathrm{D}_{2} \mathrm{O}, 1 \mathrm{H}$ ). IR: $v 1623,3344 \mathrm{~cm}^{-1}$. Anal. $\left(\mathrm{C}_{22} \mathrm{H}_{20} \mathrm{~N}_{2} \mathrm{O}_{4}(376.41)\right) \mathrm{C}, \mathrm{H}, \mathrm{N}$.

(2-(1H-Pyrazol-4-yl)-1 H-indol-3-yl)(3,4,5-trimethoxyphenyl)-methanone (15)-15 was synthesized similarly to $\mathbf{7}$, starting from $\mathbf{9 8}$. Yield $21 \%, \mathrm{mp} 112-117^{\circ} \mathrm{C}$ (from ethanol). ${ }^{1} \mathrm{H}$ NMR (DMSO- $\left.d_{6}\right): \delta 3.65(\mathrm{~s}, 6 \mathrm{H}), 3.71(\mathrm{~s}, 3 \mathrm{H}), 6.95(\mathrm{~s}, 2 \mathrm{H}), 7.06-7.10(\mathrm{~m}$, $1 \mathrm{H}), 7.16-7.20(\mathrm{~m}, 1 \mathrm{H}), 7.44-7.51(\mathrm{~m}, 2 \mathrm{H}), 7.86(\mathrm{~m}, 2 \mathrm{H}), 12.02$ (broad s, disappeared on treatment with $\left.\mathrm{D}_{2} \mathrm{O}, 1 \mathrm{H}\right), 13.04 \mathrm{ppm}$ (broad s, disappeared on treatment with $\mathrm{D}_{2} \mathrm{O}, 1 \mathrm{H}$ ). IR: $v 1634,2962,3243 \mathrm{~cm}^{-1}$. Anal. $\left(\mathrm{C}_{21} \mathrm{H}_{19} \mathrm{~N}_{3} \mathrm{O}_{4}(377.39)\right) \mathrm{C}, \mathrm{H}, \mathrm{N}$.

(2-(1 H-Imidazol-1-yl)-1 H-indol-3-yl)(3,4,5-trimethoxyphenyl)methanone (19)19 was synthesized similarly to 7 starting from $80 .{ }^{21}$ Yield $26 \%, \mathrm{mp} 210-215^{\circ} \mathrm{C}$ (from ethanol). ${ }^{1} \mathrm{H}$ NMR (DMSO- $\left.d_{6}\right): \delta 3.66(\mathrm{~s}, 3 \mathrm{H}), 3.69(\mathrm{~s}, 6 \mathrm{H}), 6.79(\mathrm{~s}, 2 \mathrm{H}), 6.88-6.89(\mathrm{~m}$, $1 \mathrm{H}), 7.24-7.32(\mathrm{~m}, 2 \mathrm{H}), 7.39-7.40(\mathrm{~m}, 1 \mathrm{H}), 7.50-7.52(\mathrm{~m}, 1 \mathrm{H}), 7.79-7.80(\mathrm{~m}, 1 \mathrm{H}), 7.97-$ $7.99(\mathrm{~m}, 1 \mathrm{H}), 12.66 \mathrm{ppm}$ (broad s, disappeared on treatment with $\left.\mathrm{D}_{2} \mathrm{O}, 1 \mathrm{H}\right)$. IR: $v 1618$, $3354 \mathrm{~cm}^{-1}$. Anal. $\left(\mathrm{C}_{21} \mathrm{H}_{19} \mathrm{~N}_{3} \mathrm{O}_{4}(377.39)\right) \mathrm{C}, \mathrm{H}, \mathrm{N}$.

(2-(Pyridin-2-yl)-1 H-indol-3-yl)(3,4,5-trimethoxyphenyl)-methanone (54)—54 was synthesized similarly to 7, starting from 92 . Yield $67 \%, \mathrm{mp} 65-70{ }^{\circ} \mathrm{C}$ (from ethanol). ${ }^{1} \mathrm{H}$ NMR (DMSO- $\left.d_{6}\right): \delta 3.61(\mathrm{~s}, 6 \mathrm{H}), 3.64(\mathrm{~s}, 3 \mathrm{H}), 6.89(\mathrm{~s}, 2 \mathrm{H}), 7.19-7.21(\mathrm{~m}, 1 \mathrm{H}), 7.26-7.28$ (m, 2H), 7.39-7.41 (m, 1H), 7.56-7.58 (m, 1H), 7.63-7.65 (m, 1H), 7.79-7.81 (m, 1H), 8.55-8.57 (m, 1H), $12.36 \mathrm{ppm}$ (broad s, disappeared on treatment with $\left.\mathrm{D}_{2} \mathrm{O}, 1 \mathrm{H}\right)$. IR: $v$ $1625,3241 \mathrm{~cm}^{-1}$. Anal. $\left(\mathrm{C}_{23} \mathrm{H}_{20} \mathrm{~N}_{2} \mathrm{O}_{4}(388.42)\right) \mathrm{C}, \mathrm{H}, \mathrm{N}$. 
(2-(Pyridin-3-yl)-1 H-indol-3-yl)(3,4,5-trimethoxyphenyl)-methanone (56)-56 was synthesized similarly to 7, starting from 93 . Yield $45 \%, \mathrm{mp} 130-135{ }^{\circ} \mathrm{C}$ (from ethanol). ${ }^{1} \mathrm{H}$ NMR (DMSO- $\left.d_{6}\right): \delta 3.60(\mathrm{~s}, 3 \mathrm{H}), 3.61(\mathrm{~s}, 6 \mathrm{H}), 6.82(\mathrm{~s}, 2 \mathrm{H}), 7.21-7.31(\mathrm{~m}, 3 \mathrm{H}), 7.53-7.55$ (m, 1H), 7.75-7.78 (m, 1H), $7.96(\mathrm{~d}, J=7.7 \mathrm{~Hz}, 1 \mathrm{H}), 8.44(\mathrm{dd}, J=1.6$ and $4.8 \mathrm{~Hz}, 1 \mathrm{H})$, 8.56-8.57 $(\mathrm{m}, 1 \mathrm{H}), 12.38 \mathrm{ppm}$ (broad s, disappeared on treatment with $\left.\mathrm{D}_{2} \mathrm{O}, 1 \mathrm{H}\right)$. IR: $v$ 1597, $3094 \mathrm{~cm}^{-1}$. Anal. $\left(\mathrm{C}_{23} \mathrm{H}_{20} \mathrm{~N}_{2} \mathrm{O}_{4}(388.42)\right) \mathrm{C}, \mathrm{H}, \mathrm{N}$.

(2-(Pyridin-4-yl)-1 $\boldsymbol{H}$-indol-3-yl)(3,4,5-trimethoxyphenyl)-methanone (58)—58 was synthesized similarly to 7 , starting from 94 . Yield $10 \%, \mathrm{mp} 50-55^{\circ} \mathrm{C}$ (from ethanol). ${ }^{1} \mathrm{H}$ NMR (DMSO- $\left.d_{6}\right): \delta 3.62(\mathrm{~s}, 3 \mathrm{H}), 3.63(\mathrm{~s}, 6 \mathrm{H}), 6.86(\mathrm{~s}, 2 \mathrm{H}), 7.21-7.25(\mathrm{~m}, 1 \mathrm{H}), 7.29-7.33$ $(\mathrm{m}, 1 \mathrm{H}), 7.35-7.37(\mathrm{~m}, 2 \mathrm{H}), 7.55-7.57(\mathrm{~m}, 1 \mathrm{H}), 7.87-7.90(\mathrm{~m}, 1 \mathrm{H}), 8.46-8.47(\mathrm{~m}, 2 \mathrm{H})$, $12.43 \mathrm{ppm}$ (broad s, disappeared on treatment with $\mathrm{D}_{2} \mathrm{O}, 1 \mathrm{H}$ ). IR: $v 1602,3304 \mathrm{~cm}^{-1}$. Anal. $\left(\mathrm{C}_{23} \mathrm{H}_{20} \mathrm{~N}_{2} \mathrm{O}_{4}(388.42)\right) \mathrm{C}, \mathrm{H}, \mathrm{N}$.

(2-(Naphthalen-1-yl)-1 H-indol-3-yl)(3,4,5-trimethoxyphenyl)methanone (60)—60 was synthesized similarly to 7 , starting from 102 . Yield $50 \%$, mp $177-179{ }^{\circ} \mathrm{C}$ (from ethanol). ${ }^{1} \mathrm{H} \mathrm{NMR}\left(\mathrm{CDCl}_{3}\right): \delta 3.40(\mathrm{~s}, 6 \mathrm{H}), 3.64(\mathrm{~s}, 3 \mathrm{H}), 6.61(\mathrm{~s}, 2 \mathrm{H}), 7.26-7.30(\mathrm{~m}, 1 \mathrm{H})$, 7.34-7.36 (m, 1H), 7.37-7.41 (m, 2H), 7.43-7.53 (m, 3H), $7.73(\mathrm{~d}, J=8.2 \mathrm{~Hz}, 1 \mathrm{H}), 7.78$ $7.83(\mathrm{~m}, 1 \mathrm{H}), 7.94-8.00(\mathrm{~m}, 1 \mathrm{H}), 8.31-8.36(\mathrm{~m}, 1 \mathrm{H}), 8.81 \mathrm{ppm}$ (broad s, disappeared on treatment with $\left.\mathrm{D}_{2} \mathrm{O}, 1 \mathrm{H}\right)$. IR: $v 1605,3158 \mathrm{~cm}^{-1}$. Anal. $\left(\mathrm{C}_{28} \mathrm{H}_{23} \mathrm{NO}_{4}(437.49)\right) \mathrm{C}, \mathrm{H}, \mathrm{N}$.

General Procedure for the Preparation of Compounds 9, 21, 23, 25, 32, 35, 40, 42, 44, 51, 63, 74, 76, and 78. Example: (2-(1-(Phenylsulfonyl)-1H-pyrrol-3-yl)-1H-indol-3-yl)(3,4,5trimethoxyphenyl)methanone (9)

A mixture of 2-(1-(phenyl-sulfonyl)- $1 H$-pyrrol-3-yl)- $1 H$-indole ${ }^{19}$ (110) $(0.26$ g, 0.00082 mol), 3,4,5-trimethoxybenzoyl chloride ( $0.23 \mathrm{~g}, 0.00098 \mathrm{~mol})$, and anhydrous aluminum chloride $(0.13 \mathrm{~g}, 0.00098 \mathrm{~mol})$ in anhydrous 1,2-dichloro-ethane $(2 \mathrm{~mL})$ was placed into the microwave cavity (closed vessel mode, $P_{\max }=250 \mathrm{psi}$ ). A starting microwave irradiation of $150 \mathrm{~W}$ was used, the temperature being ramped from 25 to $110^{\circ} \mathrm{C}$ while stirring. Once 110 ${ }^{\circ} \mathrm{C}$ was reached, taking about $1 \mathrm{~min}$, the reaction mixture was held at this temperature for 2 $\min$. The reaction mixture was quenched on $1 \mathrm{M} \mathrm{HCl} /$ crushed ice and extracted with chloroform. The organic layer was washed with brine, dried, and filtered. Removal of the solvent gave a residue that was purified by column chromatography (silica gel, ethyl acetate/ $n$-hexane $=1: 1$ as eluent) to furnish $9(0.27 \mathrm{~g}, 65 \%), \mathrm{mp} 124-126{ }^{\circ} \mathrm{C}$ (from ethanol). ${ }^{1} \mathrm{H}$ NMR (DMSO- $\left.d_{6}\right): \delta 3.57(\mathrm{~s}, 6 \mathrm{H}), 3.67(\mathrm{~s}, 3 \mathrm{H}), 6.44(\mathrm{~s}, 1 \mathrm{H}), 6.92(\mathrm{~s}, 2 \mathrm{H}), 7.07(\mathrm{t}, J=7.4$ $\mathrm{Hz}, 1 \mathrm{H}), 7.18$ (t, $J=7.4 \mathrm{~Hz}, 1 \mathrm{H}), 7.35(\mathrm{~s}, 1 \mathrm{H}), 7.43$ (d, $J=7.8 \mathrm{~Hz}, 1 \mathrm{H}), 7.49$ (d, $J=7.9 \mathrm{~Hz}$, $1 \mathrm{H}), 7.66(\mathrm{t}, J=7.8 \mathrm{~Hz}, 2 \mathrm{H}), 7.76-7.78(\mathrm{~m}, 2 \mathrm{H}), 7.96(\mathrm{~d}, J=7.4 \mathrm{~Hz}, 2 \mathrm{H}), 12.04 \mathrm{ppm}$ (broad s, disappeared on treatment with $\left.\mathrm{D}_{2} \mathrm{O}, 1 \mathrm{H}\right)$. IR: $v 1603,3303 \mathrm{~cm}^{-1}$. Anal. $\left(\mathrm{C}_{28} \mathrm{H}_{24} \mathrm{~N}_{2} \mathrm{O}_{6} \mathrm{~S}\right.$ (516.56)) C, H, N, S.

(2-(Furan-2-yl)-1 H-indol-3-yl)(3,4,5-trimethoxyphenyl)-methanone (21)-21 was synthesized similarly to $\mathbf{9}$, starting from $\mathbf{1 1 1} .{ }^{19}$ Yield $5 \%$, mp $115-117{ }^{\circ} \mathrm{C}$ (from ethanol). ${ }^{1} \mathrm{H}$ NMR $\left(\mathrm{CDCl}_{3}\right): \delta 3.80(\mathrm{~s}, 6 \mathrm{H}), 3.95(\mathrm{~s}, 3 \mathrm{H}), 6.50-6.51(\mathrm{~m}, 1 \mathrm{H}), 7.10-7.15(\mathrm{~m}$, $3 \mathrm{H}), 7.22-7.26(\mathrm{~m}, 2 \mathrm{H}), 7.41-7.46(\mathrm{~m}, 2 \mathrm{H}), 7.52-7.53(\mathrm{~m}, 1 \mathrm{H}), 9.11 \mathrm{ppm}$ (broad s, disappeared on treatment with $\left.\mathrm{D}_{2} \mathrm{O}, 1 \mathrm{H}\right)$. IR: $v 1643,3358 \mathrm{~cm}^{-1}$. Anal. $\left(\mathrm{C}_{22} \mathrm{H}_{19} \mathrm{NO}_{5}\right.$ (377.99)) C, H, N.

(2-(Furan-3-yl)-1 H-indol-3-yl)(3,4,5-trimethoxyphenyl)-methanone (23)—23 was synthesized similarly to $\mathbf{9}$, starting from $\mathbf{1 1 2} .{ }^{19}$ Yield $32 \%$, mp $115-117^{\circ} \mathrm{C}$ (from ethanol). ${ }^{1} \mathrm{H}$ NMR $\left(\mathrm{CDCl}_{3}\right): \delta 3.78(\mathrm{~s}, 6 \mathrm{H}), 3.93(\mathrm{~s}, 3 \mathrm{H}), 6.60-6.61(\mathrm{~m}, 1 \mathrm{H}), 7.13(\mathrm{~s}, 2 \mathrm{H})$, 7.15-7.19 (m, 1H), 7.25-7.27 (m, 1H), 7.43-7.46 (m, 2H), 7.58-7.60 (m, 1H), 8.05-8.06 
(m, 1H), 8.70 ppm (broad s, disappeared on treatment with $\mathrm{D}_{2} \mathrm{O}, 1 \mathrm{H}$ ). IR: $v 1598,3345$ $\mathrm{cm}^{-1}$. Anal. $\left(\mathrm{C}_{22} \mathrm{H}_{19} \mathrm{NO}_{5}(377.99)\right) \mathrm{C}, \mathrm{H}, \mathrm{N}$.

(2-(Thiophen-3-yl)-1 H-indol-3-yl)(3,4,5-trimethoxyphenyl)-methanone (25)-25 was synthesized similarly to 9 , starting from $113 .{ }^{19}$ Yield $25 \%, \mathrm{mp} 118-120{ }^{\circ} \mathrm{C}$ (from ethanol). ${ }^{1} \mathrm{H} \mathrm{NMR}\left(\mathrm{CDCl}_{3}\right): \delta 3.74(\mathrm{~s}, 6 \mathrm{H}), 3.87(\mathrm{~s}, 3 \mathrm{H}), 7.04(\mathrm{~s}, 2 \mathrm{H}), 7.11-7.12(\mathrm{~m}, 1 \mathrm{H})$, 7.23-7.28 (m, 2H), 7.29-7.33 (m, 1H), 7.44-7.47 (m, 1H), 7.50-7.51 (m, 1H), 7.91-7.93 $(\mathrm{m}, 1 \mathrm{H}), 8.60 \mathrm{ppm}$ (broad s, disappeared on treatment with $\left.\mathrm{D}_{2} \mathrm{O}, 1 \mathrm{H}\right)$. IR: $v 1606,3337$ $\mathrm{cm}^{-1}$. Anal. $\left(\mathrm{C}_{22} \mathrm{H}_{19} \mathrm{NO}_{4} \mathrm{~S}(393.46)\right) \mathrm{C}, \mathrm{H}, \mathrm{N}, \mathrm{S}$.

(2-(Oxazol-2-yl)-1 H-indol-3-yl)(3,4,5-trimethoxyphenyl)-methanone (32)-32 was synthesized similarly to $\mathbf{9}$, starting from $\mathbf{1 1 4}$. Yield $28 \%, \mathrm{mp} 208-210{ }^{\circ} \mathrm{C}$ (from ethanol). ${ }^{1} \mathrm{H}$ $\operatorname{NMR}\left(\mathrm{CDCl}_{3}\right): \delta 3.79(\mathrm{~s}, 6 \mathrm{H}), 3.94(\mathrm{~s}, 3 \mathrm{H}), 7.20-7.26(\mathrm{~m}, 4 \mathrm{H}), 7.35-7.39(\mathrm{~m}, 1 \mathrm{H}), 7.49$ $7.56(\mathrm{~m}, 2 \mathrm{H}), 7.73-7.75(\mathrm{~m}, 1 \mathrm{H}), 9.69 \mathrm{ppm}$ (broad s, disappeared on treatment with $\mathrm{D}_{2} \mathrm{O}$, 1H). IR: $v 1608,3137 \mathrm{~cm}^{-1}$. Anal. $\left(\mathrm{C}_{21} \mathrm{H}_{18} \mathrm{~N}_{2} \mathrm{O}_{5}(378.38)\right) \mathrm{C}, \mathrm{H}, \mathrm{N}$.

(2-Phenyl-1 H-indol-3-yl)(3,4,5-trimethoxyphenyl)-methanone (35)-35 was synthesized similarly to $\mathbf{9}$, starting from $\mathbf{8 4}$. Yield $95 \%$, mp $207-212{ }^{\circ} \mathrm{C}$ (from ethanol). ${ }^{1} \mathrm{H}$ NMR (DMSO- $\left.d_{6}\right): \delta 3.59(\mathrm{~s}, 3 \mathrm{H}), 3.60(\mathrm{~s}, 6 \mathrm{H}), 6.82(\mathrm{~s}, 2 \mathrm{H}), 7.18-7.28(\mathrm{~m}, 5 \mathrm{H}), 7.36-7.38$ $(\mathrm{m}, 2 \mathrm{H}), 7.50-7.53(\mathrm{~m}, 1 \mathrm{H}), 7.92-7.94(\mathrm{~m}, 1 \mathrm{H}), 12.20 \mathrm{ppm}$ (broad s, disappeared on treatment with $\left.\mathrm{D}_{2} \mathrm{O}, 1 \mathrm{H}\right)$. IR: $v 1590,3213 \mathrm{~cm}^{-1}$. Anal. $\left(\mathrm{C}_{24} \mathrm{H}_{21} \mathrm{NO}_{4}(387.43)\right) \mathrm{C}, \mathrm{H}, \mathrm{N}$.

(2-(2-Fluorophenyl)-1 H-indol-3-yl)(3,4,5-trimethoxyphenyl)-methanone (40)—40 was synthesized similarly to $\mathbf{9}$, starting from 86 . Yield $65 \%, \mathrm{mp} 180-185^{\circ} \mathrm{C}$ (from toluene). ${ }^{1} \mathrm{H}$ NMR (DMSO- $\left.d_{6}\right): \delta 3.59(\mathrm{~s}, 3 \mathrm{H}), 3.64(\mathrm{~s}, 6 \mathrm{H}), 6.79(\mathrm{~s}, 2 \mathrm{H}), 7.07-7.35(\mathrm{~m}$, $5 \mathrm{H}), 7.45-7.48(\mathrm{~m}, 1 \mathrm{H}), 7.52(\mathrm{~d}, J=8.0 \mathrm{~Hz}, 1 \mathrm{H}), 7.95(\mathrm{~d}, J=7.8 \mathrm{~Hz}, 1 \mathrm{H}), 12.27 \mathrm{ppm}$ (broad s, disappeared on treatment with $\left.\mathrm{D}_{2} \mathrm{O}, 1 \mathrm{H}\right)$. IR: $v 1591,3187 \mathrm{~cm}^{-1}$. Anal. $\left(\mathrm{C}_{24} \mathrm{H}_{20} \mathrm{FNO}_{4}\right.$ (405.42)) C, H, N, F.

(2-(3-Fluorophenyl)-1 H-indol-3-yl)(3,4,5-trimethoxyphenyl)-methanone (42)—42 was synthesized similarly to $\mathbf{9}$, starting from $\mathbf{8 7}$. Yield $52 \%$, mp $190-195{ }^{\circ} \mathrm{C}$ (from toluene). ${ }^{1} \mathrm{H}$ NMR (DMSO- $\left.d_{6}\right): \delta 3.60(\mathrm{~s}, 3 \mathrm{H}), 3.62(\mathrm{~s}, 6 \mathrm{H}), 6.78(\mathrm{~s}, 2 \mathrm{H}), 7.11(\mathrm{t}, J=8.8 \mathrm{~Hz}$, $2 \mathrm{H}), 7.20(\mathrm{t}, J=7.6 \mathrm{~Hz}, 1 \mathrm{H}), 7.27(\mathrm{t}, J=7.2 \mathrm{~Hz}, 1 \mathrm{H}), 7.39-7.42(\mathrm{~m}, 2 \mathrm{H}), 7.51(\mathrm{~d}, J=7.9$ $\mathrm{Hz}, 1 \mathrm{H}), 7.94(\mathrm{~d}, J=7.8 \mathrm{~Hz}, 1 \mathrm{H}), 12.21 \mathrm{ppm}$ (broad s, disappeared on treatment with $\mathrm{D}_{2} \mathrm{O}$, 1H). IR: $v 1594,3221 \mathrm{~cm}^{-1}$. Anal. $\left(\mathrm{C}_{24} \mathrm{H}_{20} \mathrm{FNO}_{4}(405.42)\right) \mathrm{C}, \mathrm{H}, \mathrm{F}, \mathrm{N}$.

(2-(4-Fluorophenyl)-1 H-indol-3-yl)(3,4,5-trimethoxyphenyl)-methanone (44)-44 was synthesized similarly to $\mathbf{9}$, starting from $\mathbf{8 8}$. Yield $64 \%, \mathrm{mp} 210-215^{\circ} \mathrm{C}$ (from toluene). ${ }^{1} \mathrm{H}$ NMR (DMSO- $\left.d_{6}\right): \delta 3.60(\mathrm{~s}, 3 \mathrm{H}), 3.62(\mathrm{~s}, 6 \mathrm{H}), 6.78(\mathrm{~s}, 2 \mathrm{H}), 7.10(\mathrm{t}, J=8.9 \mathrm{~Hz}$, $2 \mathrm{H}), 7.20(\mathrm{t}, J=7.3 \mathrm{~Hz}, 1 \mathrm{H}), 7.27(\mathrm{t}, J=7.1 \mathrm{~Hz}, 2 \mathrm{H}), 7.38-7.42(\mathrm{~m}, 2 \mathrm{H}), 7.51(\mathrm{~d}, J=8.2$ $\mathrm{Hz}, 1 \mathrm{H}$ ), $12.22 \mathrm{ppm}$ (broad s, disappeared on treatment with $\mathrm{D}_{2} \mathrm{O}, 1 \mathrm{H}$ ). IR: $v 1592,3218$ $\mathrm{cm}^{-1}$. Anal. $\left(\mathrm{C}_{24} \mathrm{H}_{20} \mathrm{FNO}_{4}(405.42)\right) \mathrm{C}, \mathrm{H}, \mathrm{F}, \mathrm{N}$.

(2-(4-Methoxyphenyl)-1 H-indol-3-yl)(3,4,5-trimethoxyphenyl)-methanone (51)$\mathbf{5 1}$ was synthesized similarly to $\mathbf{9}$, starting from $\mathbf{9 1}$. Yield $9 \%, \mathrm{mp} 170-175^{\circ} \mathrm{C}$ (from ethanol). ${ }^{1} \mathrm{H} \mathrm{NMR}\left(\mathrm{CDCl}_{3}\right): \delta 3.72(\mathrm{~s}, 6 \mathrm{H}), 3.78(\mathrm{~s}, 3 \mathrm{H}), 3.84(\mathrm{~s}, 3 \mathrm{H}), 6.76-6.79(\mathrm{~m}, 2 \mathrm{H})$, 7.00 (s, 2H), 7.27-7.34 (m, 4H), 7.46-7.48 (m, 1H), 8.03-8.05 (m, 1H), 8.70 ppm (broad s, disappeared on treatment with $\left.\mathrm{D}_{2} \mathrm{O}, 1 \mathrm{H}\right)$. IR: $v 1614,3144 \mathrm{~cm}^{-1}$. Anal. $\left(\mathrm{C}_{25} \mathrm{H}_{23} \mathrm{NO}_{5}\right.$ (417.45)) C, H, N.

(2-(Naphthalen-2-yl)-1 H-indol-3-yl)(3,4,5-trimethoxyphenyl)-methanone (63)$\mathbf{6 3}$ was synthesized similarly to $\mathbf{9}$, starting from $\mathbf{1 0 3}$. Yield $33 \%$, mp $182-186{ }^{\circ} \mathrm{C}$ (from 
ethanol). ${ }^{1} \mathrm{H}$ NMR $\left(\mathrm{CDCl}_{3}\right): \delta 3.56(\mathrm{~s}, 3 \mathrm{H}), 3.63(\mathrm{~s}, 6 \mathrm{H}), 6.98(\mathrm{~s}, 2 \mathrm{H}), 7.29-7.37(\mathrm{~m}, 2 \mathrm{H})$, $7.43(\mathrm{dd}, J=1.8$ and $8.5 \mathrm{~Hz}, 1 \mathrm{H}), 7.47-7.52(\mathrm{~m}, 3 \mathrm{H}), 7.68-7.78(\mathrm{~m}, 3 \mathrm{H}), 7.87(\mathrm{~m}, 1 \mathrm{H})$, $8.10-8.11(\mathrm{~m}, 1 \mathrm{H}), 8.80 \mathrm{ppm}$ (broad s, disappeared on treatment with $\mathrm{D}_{2} \mathrm{O}, 1 \mathrm{H}$ ). IR: $v 1609$, $3170 \mathrm{~cm}^{-1}$. Anal. $\left(\mathrm{C}_{28} \mathrm{H}_{23} \mathrm{NO}_{4}(437.49)\right) \mathrm{C}, \mathrm{H}, \mathrm{N}$.

(2-Cyclobutyl-1 H-indol-3-yl)(3,4,5-trimethoxyphenyl)-methanone (74)-74 was synthesized similarly to 9 , starting from 94 . Yield $75 \%$, mp $60-63{ }^{\circ} \mathrm{C}$ (from ethanol). ${ }^{1} \mathrm{H}$ NMR (DMSO- $\left.d_{6}\right): \delta 1.84-1.94(\mathrm{~m}, 2 \mathrm{H}), 2.14-2.22(\mathrm{~m}, 2 \mathrm{H}), 2.29-2.39(\mathrm{~m}, 2 \mathrm{H}), 3.76(\mathrm{~s}$, $6 \mathrm{H}), 3.77(\mathrm{~s}, 3 \mathrm{H}), 3.79-3.81(\mathrm{~m}, 1 \mathrm{H}), 6.94(\mathrm{~s}, 2 \mathrm{H}), 7.02-7.06(\mathrm{~m}, 1 \mathrm{H}), 7.12-7.16(\mathrm{~m}, 1 \mathrm{H})$, 7.40-7.42 (m, 1H), 7.44-7.47 (m, 1H), $11.95 \mathrm{ppm}$ (broad s, disappeared on treatment with $\left.\mathrm{D}_{2} \mathrm{O}, 1 \mathrm{H}\right)$. IR: $v 1600,3217 \mathrm{~cm}^{-1}$. Anal. $\left(\mathrm{C}_{22} \mathrm{H}_{23} \mathrm{NO}_{4}(365.42)\right) \mathrm{C}, \mathrm{H}, \mathrm{N}$.

(2-Cyclopentyl-1 H-indol-3-yl)(3,4,5-trimethoxyphenyl)-methanone (76)-76 was synthesized similarly to 9 , starting from 95 . Yield $68 \%, \mathrm{mp} 110-115^{\circ} \mathrm{C}$ (from ethanol). ${ }^{1} \mathrm{H}$ NMR (DMSO- $\left.d_{6}\right): \delta 1.57-1.62(\mathrm{~m}, 2 \mathrm{H}), 1.75-1.85(\mathrm{~m}, 4 \mathrm{H}), 1.95-1.99(\mathrm{~m}, 2 \mathrm{H}), 3.37-3.45$ (m, 1H), $3.76(\mathrm{~s}, 6 \mathrm{H}), 3.78(\mathrm{~s}, 3 \mathrm{H}), 6.97(\mathrm{~s}, 2 \mathrm{H}), 7.0-7.04(\mathrm{~m}, 1 \mathrm{H}), 7.10-7.14(\mathrm{~m}, 1 \mathrm{H}), 7.29-$ $7.31(\mathrm{~m}, 1 \mathrm{H}), 7.41-7.43(\mathrm{~m}, 1 \mathrm{H}), 11.77 \mathrm{ppm}$ (broad s, disappeared on treatment with $\mathrm{D}_{2} \mathrm{O}$, 1H). IR: $v 1603,3261 \mathrm{~cm}^{-1}$. Anal. $\left(\mathrm{C}_{23} \mathrm{H}_{25} \mathrm{NO}_{4}(379.45)\right) \mathrm{C}, \mathrm{H}, \mathrm{N}$.

(2-Cyclohexyl-1H-indol-3-yl)(3,4,5-trimethoxyphenyl)-methanone (78)—78 was synthesized similarly to 9 , starting from 96 . Yield $68 \%$, mp $140-145{ }^{\circ} \mathrm{C}$ (from ethanol). ${ }^{1} \mathrm{H}$ NMR (DMSO- $\left.d_{6}\right): \delta 1.08-1.26(\mathrm{~m}, 3 \mathrm{H}), 1.59-1.70(\mathrm{~m}, 3 \mathrm{H}), 1.77-1.83(\mathrm{~m}, 4 \mathrm{H}), 2.89-2.95$ (m, 1H), $3.75(\mathrm{~s}, 6 \mathrm{H}), 3.76(\mathrm{~s}, 3 \mathrm{H}), 6.95(\mathrm{~s}, 2 \mathrm{H}), 7.01-7.05(\mathrm{~m}, 1 \mathrm{H}), 7.10-7.14(\mathrm{~m}, 1 \mathrm{H})$, 7.38-7.42 (m, 2H), $11.80 \mathrm{ppm}$ (broad s, disappeared on treatment with $\left.\mathrm{D}_{2} \mathrm{O}, 1 \mathrm{H}\right) . \mathrm{IR}: v$ $1602,3285 \mathrm{~cm}^{-1}$. Anal. $\left(\mathrm{C}_{24} \mathrm{H}_{27} \mathrm{NO}_{4}(393.48)\right) \mathrm{C}, \mathrm{H}, \mathrm{N}$.

((2-(1H-Pyrrol-3-yl)-1 H-indol-3-yl)(3,4,5-trimethoxyphenyl)-methanone (8)-A mixture of $9(0.1 \mathrm{~g}, 0.00019 \mathrm{~mol})$ and $2 \mathrm{M} \mathrm{NaOH}(0.6 \mathrm{~mL})$ in methanol $(2 \mathrm{~mL})$ was heated at reflux for $3 \mathrm{~h}$. After cooling, the reaction mixture was made acidic with $2 \mathrm{M} \mathrm{HCl}(\mathrm{pH} \approx$ 3-4) and extracted with ethyl acetate. The organic layer was washed with brine, dried, and filtered. Removal of the solvent gave a residue that was purified by column chromatography (silica gel, ethyl acetate $/ n$-hexane $=1: 1$ as eluent) to furnish $8(0.7 \mathrm{~g}, 98 \%), \mathrm{mp} 114-115^{\circ} \mathrm{C}$ (from ethanol). ${ }^{1} \mathrm{H}$ NMR $\left(\mathrm{CDCl}_{3}\right): \delta 3.77(\mathrm{~s}, 6 \mathrm{H}), 3.90(\mathrm{~s}, 3 \mathrm{H}), 6.42-6.44(\mathrm{~m}, 1 \mathrm{H}), 6.77-$ $6.79(\mathrm{~m}, 1 \mathrm{H}), 7.10(\mathrm{~s}, 2 \mathrm{H}), 7.14-7.25(\mathrm{~m}, 3 \mathrm{H}), 7.40-7.42(\mathrm{~m}, 1 \mathrm{H}), 7.67-7.71(\mathrm{~m}, 1 \mathrm{H}), 8.38$ (broad s, disappeared on treatment with $\mathrm{D}_{2} \mathrm{O}, 1 \mathrm{H}$ ), $8.53 \mathrm{ppm}$ (broad s, disappeared on treatment with $\left.\mathrm{D}_{2} \mathrm{O}, 1 \mathrm{H}\right)$. IR: $v 1614,3241,3333 \mathrm{~cm}^{-1}$. Anal. $\left(\mathrm{C}_{22} \mathrm{H}_{20} \mathrm{~N}_{2} \mathrm{O}_{4}(376.41)\right) \mathrm{C}, \mathrm{H}$, N.

(2-(4,5-Dihydrooxazol-2-yl)-1H-indol-3-yl)(3,4,5-trimethoxy-phenyl)methanone (29) -29 was synthesized similarly to 28, starting from 3-(3,4,5-trimethoxybenzoyl)- $1 \mathrm{H}$ indole-2-carboxylic acid. Yield 47\%, mp 220-225 ${ }^{\circ} \mathrm{C}$ (from ethanol). ${ }^{1} \mathrm{H} \mathrm{NMR}\left(\mathrm{CDCl}_{3}\right): \delta$ $3.84(\mathrm{~s}, 6 \mathrm{H}), 3.94(\mathrm{~s}, 3 \mathrm{H}), 3.99(\mathrm{~m}, 2 \mathrm{H}), 4.19(\mathrm{~m}, 2 \mathrm{H}), 7.19(\mathrm{~s}, 2 \mathrm{H}), 7.21-7.23(\mathrm{~m}, 1 \mathrm{H})$, 7.34-7.37 (m, 1H), 7.45-7.47 (m, 1H), 7.70-7.72 (m, 1H), $10.24 \mathrm{ppm}$ (broad s, disappeared on treatment with $\left.\mathrm{D}_{2} \mathrm{O}, 1 \mathrm{H}\right)$. IR: $v 3182 \mathrm{~cm}^{-1}$. Anal. $\left(\mathrm{C}_{21} \mathrm{H}_{20} \mathrm{~N}_{2} \mathrm{O}_{5}(380.39)\right) \mathrm{C}, \mathrm{H}, \mathrm{N}$.

3-(3,4,5-Trimethoxybenzoyl)-1 H-indole-2-carboxylic Acid-A mixture of methyl 3(3,4,5-trimethoxybenzoyl)-1 $H$-indole-2-carboxylate ${ }^{18}(0.42 \mathrm{~g}, 0.00065 \mathrm{~mol})$ and $3 \mathrm{~N} \mathrm{NaOH}$ $\left(2 \mathrm{~mL}\right.$ ) was placed into the microwave cavity (closed vessel mode, $P_{\max }=250 \mathrm{psi}$ ). A starting microwave irradiation of $150 \mathrm{~W}$ was used, the temperature being ramped from 25 to $110^{\circ} \mathrm{C}$, while stirring. Once $110^{\circ} \mathrm{C}$ was reached, taking about $1 \mathrm{~min}$, the reaction mixture was held at this temperature for $2 \mathrm{~min}$, then made acidic with $3 \mathrm{~N} \mathrm{HCl}(\mathrm{pH} \approx 3-4)$ and extracted with ethyl acetate. The organic layer was washed with brine, dried, filtered, and 
evaporated to give 3-(3,4,5-trimethoxybenzoyl)- $1 H$-indole-2-carboxylic acid $(0.23 \mathrm{~g}$, 98\%). ${ }^{1} \mathrm{H}$ NMR $\left(\mathrm{CDCl}_{3}\right): \delta 3.84(\mathrm{~s}, 6 \mathrm{H}), 1.09(\mathrm{~s}, 3 \mathrm{H}), 7.11(\mathrm{~s}, 2 \mathrm{H}), 7.16-7.20(\mathrm{~m}, 1 \mathrm{H})$, 7.23-7.25 (m, 1H), 7.39-7.43 (m, 1H), $7.67(\mathrm{~m}, 1 \mathrm{H}), 10.88$ (broad s, disappeared on treatment with $\left.\mathrm{D}_{2} \mathrm{O}, 1 \mathrm{H}\right), 12.95 \mathrm{ppm}$ (broad s, disappeared on treatment with $\mathrm{D}_{2} \mathrm{O}, 1 \mathrm{H}$ ). IR: $v 1696,2837,2933,3265 \mathrm{~cm}^{-1}$. Anal. $\left(\mathrm{C}_{19} \mathrm{H}_{17} \mathrm{NO}_{6}(355.34)\right) \mathrm{C}, \mathrm{H}, \mathrm{N}$.

Arylmethyl-1 H-indoles. General Procedure for the Preparation of Compounds 12, 22, 24 , 26, 36, 52, 61, and 65. Example: 2-(1-(Phenylsulfonyl)-1 H-pyrrol-3-yl)-3-(3,4,5trimethoxybenzyl)-1 $H$-indole (12)

Borane-tetrahydrofuran complex, $1 \mathrm{M}$ solution in THF $(0.85 \mathrm{~mL}, 0.00085 \mathrm{~mol})$, was added to an ice-cooled solution of $9(0.1 \mathrm{~g}, 0.019 \mathrm{~mol})$ in acetonitrile $(1.2 \mathrm{~mL})$ containing methanol $(0.017 \mathrm{~mL})$ under an Ar stream. The reaction mixture was heated at $50{ }^{\circ} \mathrm{C}$ for $1 \mathrm{~h}$. After cooling, the reaction mixture was quenched on crushed ice and extracted with ethyl acetate. The organic layer was washed with brine, dried, and filtered. Removal of the solvent gave a residue that was purified by column chromatography ( silica gel, ethyl acetate/ $n$-hexane $=1: 1$ as eluent) to furnish $12(0.08 \mathrm{~g}, 84 \%), \mathrm{mp} 176-180{ }^{\circ} \mathrm{C}$ (from ethanol). ${ }^{1} \mathrm{H}$ NMR (DMSO$\left.d_{6}\right): \delta 3.55(\mathrm{~s}, 6 \mathrm{H}), 3.59(\mathrm{~s}, 3 \mathrm{H}), 4.15(\mathrm{~s}, 2 \mathrm{H}), 6.46(\mathrm{~s}, 2 \mathrm{H}), 6.81-6.82(\mathrm{~m}, 1 \mathrm{H}), 6.97-6.99(\mathrm{~m}$, $1 \mathrm{H}), 7.06-7.08(\mathrm{~m}, 1 \mathrm{H}), 7.32(\mathrm{~d}, J=8.0 \mathrm{~Hz}, 1 \mathrm{H}), 7.48-7.50(\mathrm{~m}, 2 \mathrm{H}), 7.62-7.66(\mathrm{~m}, 3 \mathrm{H})$, 7.75-7.76 (m, 1H), 7.94-7.96 (m, 2H), $11.16 \mathrm{ppm}$ (broad s, disappeared on treatment with $\left.\mathrm{D}_{2} \mathrm{O}, 1 \mathrm{H}\right)$. IR: $v 3293 \mathrm{~cm}^{-1}$. Anal. $\left(\mathrm{C}_{28} \mathrm{H}_{26} \mathrm{~N}_{2} \mathrm{O}_{5} \mathrm{~S}(502.58)\right) \mathrm{C}, \mathrm{H}, \mathrm{N}, \mathrm{S}$.

2-(Furan-2-yl)-3-(3,4,5-trimethoxybenzyl)-1 $\boldsymbol{H}$-indole (22)-22 was synthesized similarly to 12, starting from 21 . Yield $9 \%, \mathrm{mp} 78-80{ }^{\circ} \mathrm{C}$ (from ethanol). ${ }^{1} \mathrm{H}$ NMR $\left(\mathrm{CDCl}_{3}\right)$ : $\delta 3.76(\mathrm{~s}, 6 \mathrm{H}), 3.82(\mathrm{~s}, 3 \mathrm{H}), 4.30(\mathrm{~s}, 2 \mathrm{H}), 6.51-6.55(\mathrm{~m}, 4 \mathrm{H}), 7.09-7.13(\mathrm{~m}, 1 \mathrm{H}), 7.21-7.24$ (m, 1H), 7.40-7.43 (m, 1H), 7.52-7.54 (m, 2H), $8.49 \mathrm{ppm}$ (broad s, disappeared on treatment with $\left.\mathrm{D}_{2} \mathrm{O}, 1 \mathrm{H}\right)$. IR: $v 3347 \mathrm{~cm}^{-1}$. Anal. $\left(\mathrm{C}_{22} \mathrm{H}_{21} \mathrm{NO}_{4}(363.41)\right) \mathrm{C}, \mathrm{H}, \mathrm{N}$.

2-(Furan-3-yl)-3-(3,4,5-trimethoxybenzyl)-1 $\mathrm{H}$-indole (24)-24 was synthesized similarly to $\mathbf{1 2}$, starting from $\mathbf{2 3}$. Yield $13 \%, \mathrm{mp} 102-105{ }^{\circ} \mathrm{C}$ (from ethanol). ${ }^{1} \mathrm{H}$ NMR $\left(\mathrm{CDCl}_{3}\right): \delta 3.73(\mathrm{~s}, 6 \mathrm{H}), 3.80(\mathrm{~s}, 3 \mathrm{H}), 4.18(\mathrm{~s}, 2 \mathrm{H}), 6.44(\mathrm{~s}, 2 \mathrm{H}), 6.65-6.66(\mathrm{~m}, 1 \mathrm{H}), 7.07-$ $7.11(\mathrm{~m}, 1 \mathrm{H}), 7.17-7.19(\mathrm{~m}, 1 \mathrm{H}), 7.37-7.39(\mathrm{~m}, 1 \mathrm{H}), 7.47-7.49(\mathrm{~m}, 1 \mathrm{H}), 7.52-7.53(\mathrm{~m}, 1 \mathrm{H})$, 7.63-7.64 (m, 1H), $8.07 \mathrm{ppm}$ (broad s, disappeared on treatment with $\mathrm{D}_{2} \mathrm{O}, 1 \mathrm{H}$ ). IR: $v 3320$ $\mathrm{cm}^{-1}$. Anal. $\left(\mathrm{C}_{22} \mathrm{H}_{21} \mathrm{NO}_{4}(363.41)\right) \mathrm{C}, \mathrm{H}, \mathrm{N}$.

2-(Thiophen-3-yl)-3-(3,4,5-trimethoxybenzyl)-1 H-indole (26)-26 was synthesized similarly to $\mathbf{1 2}$, starting from 25 . Yield $61 \%$, mp $165-168{ }^{\circ} \mathrm{C}$ (from ethanol). ${ }^{1} \mathrm{H}$ NMR $\left(\mathrm{CDCl}_{3}\right): \delta 3.74(\mathrm{~s}, 6 \mathrm{H}), 3.83(\mathrm{~s}, 3 \mathrm{H}), 4.25(\mathrm{~s}, 2 \mathrm{H}), 6.48(\mathrm{~s}, 2 \mathrm{H}), 7.10-7.14(\mathrm{~m}, 1 \mathrm{H}), 7.21-$ $7.25(\mathrm{~m}, 1 \mathrm{H}), 7.34-7.35(\mathrm{~m}, 1 \mathrm{H}), 7.40-7.46(\mathrm{~m}, 3 \mathrm{H}), 7.51-7.53(\mathrm{~m}, 1 \mathrm{H}), 8.22 \mathrm{ppm}$ (broad s, disappeared on treatment with $\left.\mathrm{D}_{2} \mathrm{O}, 1 \mathrm{H}\right)$. IR: $v 3353 \mathrm{~cm}^{-1}$. Anal. $\left(\mathrm{C}_{22} \mathrm{H}_{21} \mathrm{NO}_{3} \mathrm{~S}(379.47)\right)$ $\mathrm{C}, \mathrm{H}, \mathrm{N}, \mathrm{S}$.

2-Phenyl-3-(3,4,5-trimethoxybenzyl)-1 $\boldsymbol{H}$-indole (36)-36 was synthesized similarly to 12, starting from 35 . Yield $26 \%, \mathrm{mp} 187-192{ }^{\circ} \mathrm{C}$ (from ethanol). ${ }^{1} \mathrm{H}$ NMR (DMSO- $d_{6}$ ): $\delta$ $3.59(\mathrm{~s}, 3 \mathrm{H}), 3.60(\mathrm{~s}, 6 \mathrm{H}), 4.17(\mathrm{~s}, 2 \mathrm{H}), 6.46(\mathrm{~s}, 2 \mathrm{H}), 6.97-7.01(\mathrm{~m}, 1 \mathrm{H}), 7.09-7.13(\mathrm{~m}, 1 \mathrm{H})$, 7.37-7.41 (m, 2H), 7.47-7.53 (m, 3H), 7.64-7.66 (m, 2H), $11.28 \mathrm{ppm}$ (broad s, disappeared on treatment with $\left.\mathrm{D}_{2} \mathrm{O}, 1 \mathrm{H}\right)$. IR: $v 3351 \mathrm{~cm}^{-1}$. Anal. $\left(\mathrm{C}_{24} \mathrm{H}_{23} \mathrm{NO}_{3}(373.44)\right) \mathrm{C}, \mathrm{H}, \mathrm{N}$.

2-(4-Methoxyphenyl)-3-(3,4,5-trimethoxybenzyl)-1 $\boldsymbol{H}$-indole (52)—52 was synthesized similarly to $\mathbf{1 2}$, starting from $\mathbf{5 1}$. Yield $4 \%$ as an oil. ${ }^{1} \mathrm{H}$ NMR $\left(\mathrm{CDCl}_{3}\right): \delta 3.73$ (s, 6H), 3.82 (s, 3H), 3.86 (s, 3H), 4.19 (s, 2H), 6.46 (s, 2H), 6.98-7.00 (m, 2H), 7.07-7.11 (m, 1H), 7.18-7.22 (m, 1H), 7.39-7.41 (m, 1H), 7.46-7.49 (m, 3H), 8.09 ppm (broad s, 
disappeared on treatment with $\left.\mathrm{D}_{2} \mathrm{O}, 1 \mathrm{H}\right)$. IR: $v 3356 \mathrm{~cm}^{-1}$. Anal. $\left(\mathrm{C}_{25} \mathrm{H}_{25} \mathrm{NO}_{4}(403.47)\right) \mathrm{C}$, $\mathrm{H}, \mathrm{N}$.

2-(Naphthalen-1-yl)-3-(3,4,5-trimethoxybenzyl)-1H-indole (61)-61 was synthesized similarly to $\mathbf{1 2}$, starting from $\mathbf{6 0}$. Yield 99\%, mp $142-147{ }^{\circ} \mathrm{C}$ (from ethanol). ${ }^{1} \mathrm{H}$ NMR $\left(\mathrm{CDCl}_{3}\right): \delta 3.60(\mathrm{~s}, 6 \mathrm{H}), 3.74(\mathrm{~s}, 3 \mathrm{H}), 4.03(\mathrm{~s}, 2 \mathrm{H}), 6.26(\mathrm{~s}, 2 \mathrm{H}), 7.18-7.20(\mathrm{~m}, 1 \mathrm{H})$, 7.25-7.29 (m, 1H), 7.43-7.47 (m, 2H), 7.52-7.58 (m, 3H), 7.63-7.65 (m, 1H), 7.84-7.86 $(\mathrm{m}, 1 \mathrm{H}), 7.94-7.96(\mathrm{~m}, 2 \mathrm{H}), 8.19 \mathrm{ppm}$ (broad s, disappeared on treatment with $\left.\mathrm{D}_{2} \mathrm{O}, 1 \mathrm{H}\right)$. IR: $v 3317 \mathrm{~cm}^{-1}$. Anal. $\left(\mathrm{C}_{28} \mathrm{H}_{25} \mathrm{NO}_{3}(423.50)\right) \mathrm{C}, \mathrm{H}, \mathrm{N}$.

2-(Naphthalen-2-yl)-3-(3,4,5-trimethoxybenzyl)-1 $H$-indole (65)-65 was synthesized similarly to $\mathbf{1 2}$, starting from $\mathbf{6 3}$. Yield $73 \%$, mp $138-140{ }^{\circ} \mathrm{C}$ (from ethanol). ${ }^{1} \mathrm{H}$ $\operatorname{NMR}\left(\mathrm{CDCl}_{3}\right): \delta 3.73(\mathrm{~s}, 6 \mathrm{H}), 3.83(\mathrm{~s}, 3 \mathrm{H}), 4.29(\mathrm{~s}, 2 \mathrm{H}), 6.52(\mathrm{~s}, 2 \mathrm{H}), 7.13-7.17(\mathrm{~m}, 1 \mathrm{H})$, $7.24-7.28(\mathrm{~m}, 1 \mathrm{H}), 7.46(\mathrm{~d}, J=8.1 \mathrm{~Hz}, 1 \mathrm{H}), 7.51-7.58(\mathrm{~m}, 3 \mathrm{H}), 7.70(\mathrm{dd}, J=1.8$ and 8.5 $\mathrm{Hz}, 1 \mathrm{H}), 7.82-7.94(\mathrm{~m}, 3 \mathrm{H}), 7.99(\mathrm{~m}, 1 \mathrm{H}), 8.30 \mathrm{ppm}$ (broad s, disappeared on treatment with $\left.\mathrm{D}_{2} \mathrm{O}, 1 \mathrm{H}\right)$. IR: $v 3359 \mathrm{~cm}^{-1}$. Anal. $\left(\mathrm{C}_{28} \mathrm{H}_{25} \mathrm{NO}_{3}(423.50)\right) \mathrm{C}, \mathrm{H}, \mathrm{N}$.

2-(1 H-Pyrrol-3-yl)-3-(3,4,5-trimethoxybenzyl)-1 $\boldsymbol{H}$-indole (13)-13 was synthesized similarly to 8, from $\mathbf{1 2}$. Yield $20 \%, \mathrm{mp} 195-197{ }^{\circ} \mathrm{C}$ (from ethanol). ${ }^{1} \mathrm{H}$ NMR (DMSO- $d_{6}$ ): $\delta$ 3.59 (s, 3H), 3.61 (s, 6H), $4.14(\mathrm{~s}, 2 \mathrm{H}), 6.48-6.49(\mathrm{~m}, 1 \mathrm{H}), 6.53(\mathrm{~s}, 2 \mathrm{H}), 6.86-6.92(\mathrm{~m}, 2 \mathrm{H})$, 6.97-7.01 (m, 1H), 7.12-7.13 (m, 1H), 7.29 (d, $J=7.8 \mathrm{~Hz}, 1 \mathrm{H}), 7.37(\mathrm{~d}, J=7.7 \mathrm{~Hz}, 1 \mathrm{H})$, 10.88 (broad s, disappeared on treatment with $\mathrm{D}_{2} \mathrm{O}, 1 \mathrm{H}$ ), $11.03 \mathrm{ppm}$ (broad s, disappeared on treatment with $\left.\mathrm{D}_{2} \mathrm{O}, 1 \mathrm{H}\right)$. IR: $v 3242 \mathrm{~cm}^{-1}$. Anal. $\left(\mathrm{C}_{22} \mathrm{H}_{22} \mathrm{~N}_{2} \mathrm{O}_{3}(362.42)\right) \mathrm{C}, \mathrm{H}, \mathrm{N}$.

\section{2-(3-(3,4,5-Trimethoxybenzyl)-1H-indol-2-yl)-4,5-dihydroxazole (30)-30 was} synthesized similarly to $\mathbf{2 8}$, starting from 3-(3,4,5-tri-methoxybenzyl)- $1 H$-indole-2carboxylic acid. Yield $80 \%, \mathrm{mp} 160-165{ }^{\circ} \mathrm{C}$ (from ethanol). ${ }^{1} \mathrm{H} \mathrm{NMR}\left(\mathrm{CDCl}_{3}\right): \delta 3.77$ (s, $6 \mathrm{H}), 3.80(\mathrm{~s}, 3 \mathrm{H}), 4.09(\mathrm{t}, J=9.5 \mathrm{~Hz}, 2 \mathrm{H}), 4.44-4.52(\mathrm{~m}, 4 \mathrm{H}), 6.55(\mathrm{~s}, 2 \mathrm{H}), 7.07-7.11(\mathrm{~m}$, $1 \mathrm{H}), 7.25-7.29(\mathrm{~m}, 1 \mathrm{H}), 7.36(\mathrm{dd}, J=0.75$ and $8.3 \mathrm{~Hz}, 1 \mathrm{H}), 7.56(\mathrm{~d}, J=8.2 \mathrm{~Hz}, 1 \mathrm{H}), 9.10$ ppm (broad s, disappeared on treatment with $\mathrm{D}_{2} \mathrm{O}, 1 \mathrm{H}$ ). IR: $v 3257 \mathrm{~cm}^{-1}$. Anal. $\left(\mathrm{C}_{21} \mathrm{H}_{22} \mathrm{~N}_{2} \mathrm{O}_{4}(366.41)\right) \mathrm{C}, \mathrm{H}, \mathrm{N}$.

3-(3,4,5-Trimethoxybenzyl)-1H-indole-2-carboxylic Acid-The compound was obtained from methyl 3-(3,4,5-trimethoxybenzyl)-1 $H$-indole-2-carboxylate. ${ }^{18}$ Yield $96 \%$, $\mathrm{mp} 240-245^{\circ} \mathrm{C}$ (from ethanol). ${ }^{1} \mathrm{H}$ NMR (DMSO- $\left.d_{6}\right): \delta 3.56(\mathrm{~s}, 3 \mathrm{H}), 3.66(\mathrm{~s}, 6 \mathrm{H}), 4.37(\mathrm{~s}$, 2H), 6.65 (s, 2H), 6.99-7.03 (m, 1H), 7.18-7.23 (m, 1H), $7.38(\mathrm{~d}, J=8.0 \mathrm{~Hz}, 1 \mathrm{H}), 7.67$ (d, $J$ $=8.0 \mathrm{~Hz}, 1 \mathrm{H}), 11.48\left(\right.$ broad $\mathrm{s}$, disappeared on treatment with $\left.\mathrm{D}_{2} \mathrm{O}, 1 \mathrm{H}\right), 13.05 \mathrm{ppm}$ (broad s, disappeared on treatment with $\left.\mathrm{D}_{2} \mathrm{O}, 1 \mathrm{H}\right)$. IR: $v 1663,2840,3354 \mathrm{~cm}^{-1}$. Anal. $\left(\mathrm{C}_{19} \mathrm{H}_{19} \mathrm{NO}_{5}\right.$ (341.36)) C, H, N.

\section{Arylmethanol-1 H-indoles. (2-(1 H-Pyrrol-3-yl)-1 H-indol-3-yl)-(3,4,5- trimethoxyphenyl)methanol (10)}

$\mathbf{1 0}$ was synthesized similarly to $\mathbf{8}$, starting from $\mathbf{1 1}$. Yield $98 \%$, mp $189-191{ }^{\circ} \mathrm{C}$ (from ethanol). ${ }^{1} \mathrm{H}$ NMR $\left(\mathrm{CDCl}_{3}\right): \delta 3.57(\mathrm{~s}, 6 \mathrm{H}), 3.86(\mathrm{~s}, 3 \mathrm{H}), 5.13(\mathrm{~m}, 1 \mathrm{H}), 6.00$ (s, disappeared on treatment with $\left.\mathrm{D}_{2} \mathrm{O}, 1 \mathrm{H}\right), 6.38-6.39(\mathrm{~m}, 2 \mathrm{H}), 6.54(\mathrm{~s}, 1 \mathrm{H}), 6.62-6.64(\mathrm{~m}, 1 \mathrm{H}), 6.75-6.78$ $(\mathrm{m}, 3 \mathrm{H}), 6.85-6.88(\mathrm{~m}, 1 \mathrm{H}), 7.00-7.03(\mathrm{~m}, 1 \mathrm{H}), 8.01$ (broad s, disappeared on treatment with $\mathrm{D}_{2} \mathrm{O}, 1 \mathrm{H}$ ), $8.16 \mathrm{ppm}$ (broad s, disappeared on treatment with $\mathrm{D}_{2} \mathrm{O}, 1 \mathrm{H}$ ). IR: $v 2852$, 2922, $3382 \mathrm{~cm}^{-1}$. Anal. $\left(\mathrm{C}_{22} \mathrm{H}_{22} \mathrm{~N}_{2} \mathrm{O}_{4}(378.42)\right) \mathrm{C}, \mathrm{H}, \mathrm{N}$. 
General Procedure for the Preparation of Compounds 11 and 64. Example: (2-(1(Phenylsulfonyl)-1 H-pyrrol-3-yl)-1 H-indol-3-yl)(3,4,5-trimethoxyphenyl)methanol (11)

To a solution of $9(0.5 \mathrm{~g}, 0.0013 \mathrm{~mol})$ in THF $(2.6 \mathrm{~mL})$ containing $0.08 \mathrm{~mL}$ of water was carefully added sodium borohydride $(0.049 \mathrm{~g}, 0.0013 \mathrm{~mol})$. The reaction mixture was heated at $80^{\circ} \mathrm{C}$ for $2 \mathrm{~h}$. After cooling, the mixture was diluted with water and extracted with ethyl acetate. The organic layer was washed with brine, dried, and filtered. Removal of the solvent gave a residue that was purified by column chromatography (silica gel, acetone $/ n$-hexane $=$ 1:1 as eluent) to furnish $11(0.54 \mathrm{~g}, 97 \%), \mathrm{mp} 90-94{ }^{\circ} \mathrm{C}$ (from ethanol). ${ }^{1} \mathrm{H}$ NMR (DMSO$\left.d_{6}\right): 3.58(\mathrm{~s}, 6 \mathrm{H}), 3.60(\mathrm{~s}, 3 \mathrm{H}), 5.71-5.73(\mathrm{~m}, 1 \mathrm{H}), 6.05$ (s, disappeared on treatment with $\left.\mathrm{D}_{2} \mathrm{O}, 1 \mathrm{H}\right), 6.63(\mathrm{~s}, 2 \mathrm{H}), 6.83-6.86(\mathrm{~m}, 2 \mathrm{H}), 7.01(\mathrm{t}, J=6.6 \mathrm{~Hz}, 1 \mathrm{H}), 7.27(\mathrm{~d}, J=8.4 \mathrm{~Hz}, 1 \mathrm{H})$, $7.36(\mathrm{~d}, J=7.4 \mathrm{~Hz}, 1 \mathrm{H}), 7.47-7.50(\mathrm{~m}, 1 \mathrm{H}), 7.63-7.67(\mathrm{~m}, 2 \mathrm{H}), 7.74-7.77(\mathrm{~m}, 2 \mathrm{H}), 7.98(\mathrm{~d}$, $J=7.1 \mathrm{~Hz}, 2 \mathrm{H}$ ), $11.14 \mathrm{ppm}$ (broad s, disappeared on treatment with $\mathrm{D}_{2} \mathrm{O}, 1 \mathrm{H}$ ). IR: $v 2938$, $3341 \mathrm{~cm}^{-1}$. Anal. $\left(\mathrm{C}_{28} \mathrm{H}_{26} \mathrm{~N}_{2} \mathrm{O}_{6} \mathrm{~S}(518.58)\right) \mathrm{C}, \mathrm{H}, \mathrm{N}, \mathrm{S}$.

(2-(Naphthalen-2-yl)-1 H-indol-3-yl)(3,4,5-trimethoxyphenyl)methanol (64)-64 was synthesized similarly to $\mathbf{1 1}$, starting from $\mathbf{6 3}$. Yield $44 \%, \mathrm{mp} 145-150{ }^{\circ} \mathrm{C}$ (from ethanol). ${ }^{1} \mathrm{H}$ NMR $\left(\mathrm{CDCl}_{3}\right): \delta 2.29(\mathrm{~d}, J=4.2 \mathrm{~Hz}, 1 \mathrm{H}), 3.76(\mathrm{~s}, 6 \mathrm{H}), 3.84(\mathrm{~s}, 3 \mathrm{H}), 6.29(\mathrm{~d}, J$ $=4.2 \mathrm{~Hz}, 1 \mathrm{H}), 6.79(\mathrm{~s}, 2 \mathrm{H}), 7.07-7.11(\mathrm{~m}, 1 \mathrm{H}), 7.22-7.26(\mathrm{~m}, 1 \mathrm{H}), 7.43-7.46(\mathrm{~m}, 1 \mathrm{H})$, 7.55-7.60 (m, 3H), $7.72(\mathrm{dd}, J=1.8$ and $8.5 \mathrm{~Hz}, 1 \mathrm{H}), 7.87-7.91(\mathrm{~m}, 2 \mathrm{H}), 7.96(\mathrm{~d}, J=8.4$ $\mathrm{Hz}, 1 \mathrm{H}), 8.06(\mathrm{~m}, 1 \mathrm{H}), 8.35 \mathrm{ppm}$ (broad s, disappeared on treatment with $\left.\mathrm{D}_{2} \mathrm{O}, 1 \mathrm{H}\right)$. IR: $v$ $3286,3295,3293 \mathrm{~cm}^{-1}$. Anal. $\left(\mathrm{C}_{28} \mathrm{H}_{25} \mathrm{NO}_{4}(439.50)\right) \mathrm{C}, \mathrm{H}, \mathrm{N}$.

Synthetic Intermediates. 2-(1H-Imidazol-2-yl)-1 $\mathrm{H}$-indole (79). (Diacetoxyiodo)benzene $(0.52 \mathrm{~g}, 0.0016 \mathrm{~mol})$ was added to a mixture of 2-(4,5-dihydro- $1 H$-imidazol-2-yl)- $1 H$-indole (83) $(0.27 \mathrm{~g}, 0.0015 \mathrm{~mol})$ and potassium carbonate $(0.22 \mathrm{~g}, 0.0016 \mathrm{~mol})$ in DMSO $(15 \mathrm{~mL})$. The reaction mixture was stirred at $25^{\circ} \mathrm{C}$ for $12 \mathrm{~h}$ under an Ar stream, then diluted with a saturated aqueous solution of sodium hydrogen carbonate and extracted with ethyl acetate. The organic layer was washed with brine, dried, and filtered. Removal of the solvent gave a residue that was purified by column chromatography (silica gel, ethyl acetate/ $n$-hexane $=1: 1$ as eluent) to furnish $79(0.08 \mathrm{~g}, 29 \%), \mathrm{mp} 130-133{ }^{\circ} \mathrm{C}$ (from ethanol). ${ }^{1} \mathrm{H}$ NMR (DMSO$\left.d_{6}\right): \delta 7.12(\mathrm{t}, J=7.5 \mathrm{~Hz}, 1 \mathrm{H}), 7.20(\mathrm{t}, J=7.4 \mathrm{~Hz}, 1 \mathrm{H}), 7.23-7.25(\mathrm{~m}, 3 \mathrm{H}), 7.32(\mathrm{~d}, J=7.8$ $\mathrm{Hz}, 1 \mathrm{H}), 7.41(\mathrm{~d}, J=7.8 \mathrm{~Hz}, 1 \mathrm{H}), 11.90$ (broad s, disappeared on treatment with $\left.\mathrm{D}_{2} \mathrm{O}, 1 \mathrm{H}\right)$, $12.19 \mathrm{ppm}$ (broad s, disappeared on treatment with $\mathrm{D}_{2} \mathrm{O}, 1 \mathrm{H}$ ). IR: $v 2920,3060,3120,3380$ $\mathrm{cm}^{-1}$.

2-(1H-1,2,4-Triazol-1-yl)-1 H-indole (81)—A mixture of 2-iodo- $1 H$-indole ${ }^{23}$ (106) $(0.5$ $\mathrm{g}, 0.0021 \mathrm{~mol})$ and triazole $(0.14 \mathrm{~g}, 0.021 \mathrm{~mol})$ was heated at $150^{\circ} \mathrm{C}$ for $2 \mathrm{~h}$. After cooling, the mixture was purified by column chromatography (silica gel, ethyl acetate as eluent) to give $81(0.16 \mathrm{~g}, 42 \%)$ as a powder. ${ }^{1} \mathrm{H}$ NMR (DMSO- $\left.d_{6}\right): \delta 6.76(\mathrm{~s}, 1 \mathrm{H}), 7.08(\mathrm{t}, J=7.2 \mathrm{~Hz}$, $1 \mathrm{H}), 7.17(\mathrm{t}, J=7.1 \mathrm{~Hz}, 1 \mathrm{H}), 7.43(\mathrm{~d}, J=8.3 \mathrm{~Hz}, 1 \mathrm{H}), 7.58(\mathrm{~d}, J=7.9 \mathrm{~Hz}, 1 \mathrm{H}), 8.32(\mathrm{~s}, 1 \mathrm{H})$, 9.22 (broad s, disappeared on treatment with $\mathrm{D}_{2} \mathrm{O}, 1 \mathrm{H}$ ), $12.19 \mathrm{ppm}$ (broad s, disappeared on treatment with $\left.\mathrm{D}_{2} \mathrm{O}, 1 \mathrm{H}\right)$. IR: $v 3112 \mathrm{~cm}^{-1}$.

2-(1 H-Indol-2-yl)thiazole (82)—A mixture 2-bromo-1-(thiazol-2-yl)ethanone (122) (0.5 $\mathrm{g}, 0.0024 \mathrm{~mol})$ and aniline $(0.67 \mathrm{~g}, 0.6 \mathrm{~mL}, 0.0072 \mathrm{~mol})$ in anhydrous DMF $(2 \mathrm{~mL})$ was placed into the microwave cavity (closed vessel mode, $P_{\max }=250 \mathrm{psi}$ ). A starting microwave irradiation of $100 \mathrm{~W}$ was used, the temperature being ramped from 25 to $150{ }^{\circ} \mathrm{C}$, while stirring. Once $150{ }^{\circ} \mathrm{C}$ was reached, taking about $1 \mathrm{~min}$, the reaction mixture was held at this temperature for $1 \mathrm{~min}$, while cooling, then diluted with water and extracted with ethyl acetate. The organic layer was washed with brine, dried, and filtered. Removal of the solvent gave a residue that was purified by column chromatography (silica gel, ethyl acetate/nhexane $=2: 7$ as eluent) to furnish $82(0.25 \mathrm{~g}, 52 \%), \mathrm{mp} 112-115^{\circ} \mathrm{C}$ (from ethanol). 
2-(4,5-Dihydro-1 $\boldsymbol{H}$-imidazol-2-yl)-1 $\boldsymbol{H}$-indole (83)—Ethylenediamine $(0.23 \mathrm{~g}, 0.25 \mathrm{~mL}$, $0.0038 \mathrm{~mol})$ was added to a solution of $1 H$-indole-2-carbaldehyde ${ }^{26}(\mathbf{1 1 5})(0.5 \mathrm{~g}, 0.0034$ $\mathrm{mol})$ in tert-butanol $(34 \mathrm{~mL})$. The reaction mixture was stirred at $25^{\circ} \mathrm{C}$ for 30 min under an Ar stream. Iodine $(1.08 \mathrm{~g}, 0.00425 \mathrm{~mol})$ and potassium carbonate $(1.41 \mathrm{~g}, 0.01 \mathrm{~mol})$ were added, and the reaction mixture was heated at $70{ }^{\circ} \mathrm{C}$ for $3 \mathrm{~h}$. After cooling, the mixture was diluted with a saturated aqueous solution of sodium sulfite and extracted with chloroform. The organic layer was washed with brine, dried, and filtered. Removal of the solvent gave a residue that was purified by column chromatography (silica gel, chloroform/ethanol $=8: 2$ as eluent) to furnish $83(0.27 \mathrm{~g}, 43 \%) .{ }^{1} \mathrm{H}$ NMR (D MSO- $\left.d_{6}\right): \delta 3.70(\mathrm{~s}, 4 \mathrm{H}), 7.13(\mathrm{t}, J=7.6$ $\mathrm{Hz}, 1 \mathrm{H}), 7.25(\mathrm{t}, J=6.9 \mathrm{~Hz}, 1 \mathrm{H}), 7.35(\mathrm{~d}, J=7.8 \mathrm{~Hz}, 1 \mathrm{H}), 7.42(\mathrm{~d}, J=8.2 \mathrm{~Hz}, 1 \mathrm{H}), 8.68$ (broad s, disappeared on treatment with $\mathrm{D}_{2} \mathrm{O}, 1 \mathrm{H}$ ), $11.79 \mathrm{ppm}$ (broad s, disappeared on treatment with $\left.\mathrm{D}_{2} \mathrm{O}, 1 \mathrm{H}\right)$. IR: $v 3387,2855 \mathrm{~cm}^{-1}$.

\section{General Procedure for the Preparation of Compounds 85, 91-94, and 103. Example: 2-(4-} Chlorophenyl)-1 $\mathrm{H}$-indole (85)

1-(1-(4-Chlorophenyl)ethylidene)-2-phenylhydrazine (128) (2.82 g, $0.011 \mathrm{~mol})$ was added in portions to polyphosphoric acid $(28 \mathrm{~g})$ at $120^{\circ} \mathrm{C}$. The reaction mixture was stirred at the same temperature for $1 \mathrm{~h}$. After cooling, it was quenched on crushed ice and treated with 3 $\mathrm{N} \mathrm{NaOH}$ with stirring, then extracted with ethyl acetate. The organic layer was washed with brine, dried, and filtered. Removal of the solvent gave a residue that was purified by column chromatography (silica gel, ethyl acetate $/ n$-hexane $=1: 1$ as eluent) to furnish $\mathbf{8 5}(2.1 \mathrm{~g}$, $80 \%$ ), mp $210-212{ }^{\circ} \mathrm{C}$ (from ethanol). Lit. ${ }^{37} \mathrm{mp} 206.3-209.0^{\circ} \mathrm{C}$.

2-(4-Methoxyphenyl)-1 $\mathrm{H}$-indole (91)—91 was synthesized similarly to 85 , starting from 1-(1-(4-methoxyphenyl)ethylidene)-2-phenylhydrazine (129). Yield 37\%, mp 222-224 ${ }^{\circ} \mathrm{C}$ (from toluene). Lit. ${ }^{38} \mathrm{mp} 224-227^{\circ} \mathrm{C}$.

2-(Pyridin-2-yl)-1 H-indole (92)—92 was synthesized similarly to 85, starting from 2-(1(2-phenylhydrazono)ethyl)pyridine ${ }^{29}$ (130). Yield $73 \%, \mathrm{mp} 154-156^{\circ} \mathrm{C}$ (from ethanol). Lit. ${ }^{39} \mathrm{mp} 156.0-156.5^{\circ} \mathrm{C}$.

2-(Pyridin-3-yl)-1 H-indole (93)—93 was synthesized similarly to 85, starting from 3-(1(2-phenylhydrazono)ethyl)pyridine ${ }^{29}(\mathbf{1 3 1})$. Yield $73 \%, \mathrm{mp} 168-170{ }^{\circ} \mathrm{C}$ (from ethanol). Lit. ${ }^{40} \mathrm{mp} 169-170{ }^{\circ} \mathrm{C}$.

2-(Pyridin-4-yl)-1 $\mathbf{H}$-indole (94) - 94 was synthesized similarly to 85 , starting from 4-(1(2-phenylhydrazono)ethyl)pyridine ${ }^{29}$ (132). Yield 68\%, mp 237-239 ${ }^{\circ} \mathrm{C}$ (from ethanol). Lit. ${ }^{41} \mathrm{mp} 238-239^{\circ} \mathrm{C}$.

2-(Naphthalen-2-yl)-1 H-indole (103)-103 was synthesized similarly to 85, starting from 1-(1-(naphthalen-2-yl)ethylidene)-2-phenylhydrazine ${ }^{29}$ (133). Yield 45\%, mp 165-168 ${ }^{\circ} \mathrm{C}$ (petroleum ether). Lit. ${ }^{42} \mathrm{mp} 163-165^{\circ} \mathrm{C}$.

General Procedure for the Preparation of Compounds 86-90, 101, and 102. Example: 2-(2Fluorophenyl)- $1 \mathrm{H}$-indole (86)

A solution of bromo-1-(2-fluorophenyl)ethanone (116) $(0.9 \mathrm{~g}, 0.0041)$ in $N, N$ dimethylaniline $(1.8 \mathrm{~mL})$ was added dropwise to aniline $(1.26 \mathrm{~g}, 1.2 \mathrm{~mL}, 0.0135 \mathrm{~mol})$ in the same solvent $(2.4 \mathrm{~mL})$. The reaction mixture was heated at $170{ }^{\circ} \mathrm{C}$ for $15 \mathrm{~min}$. After cooling to $0{ }^{\circ} \mathrm{C}$, the mixture was made acidic with $1 \mathrm{M} \mathrm{HCl}(\mathrm{pH} \approx 3-4)$ and extracted with ethyl acetate. The organic layer was washed with brine, dried, and filtered. Removal of the solvent gave a residue that was purified by column chromatography (silica gel, acetone $/ n$-hexane $=$ 
1:3 as eluent) to furnish $89(0.3 \mathrm{~g}, 35 \%)$, mp $102-104{ }^{\circ} \mathrm{C}$ (from toluene). ${ }^{1} \mathrm{H}$ NMR (DMSO$\left.d_{6}\right): \delta 6.92(\mathrm{~s}, 1 \mathrm{H}), 7.03(\mathrm{t}, J=7.8 \mathrm{~Hz}, 1 \mathrm{H}), 7.14(\mathrm{t}, J=7.8 \mathrm{~Hz}, 1 \mathrm{H}), 7.34-7.40(\mathrm{~m}, 3 \mathrm{H}), 7.45$ $(\mathrm{d}, J=8.0 \mathrm{~Hz}, 1 \mathrm{H}), 7.58(\mathrm{~d}, J=7.9 \mathrm{~Hz}, 1 \mathrm{H}), 7.93(\mathrm{t}, J=7.6 \mathrm{~Hz}, 1 \mathrm{H}), 11.48 \mathrm{ppm}$ (broad s, disappeared on treatment with D2O, 1H). IR: $v 3412 \mathrm{~cm}^{-1}$. Lit. ${ }^{43} \mathrm{mp} 97-98.5^{\circ} \mathrm{C}$.

2-(3-Fluorophenyl)-1 $\mathbf{H}$-indole (87)—87 was synthesized similarly to $\mathbf{8 6}$, starting from 2-bromo-1-(3-fluorophenyl)ethanone (117). Yield 42\%, mp 142-144 ${ }^{\circ} \mathrm{C}$ (from toluene). Lit. ${ }^{44} \mathrm{mp} 144{ }^{\circ} \mathrm{C}$.

2-(4-Fluorophenyl)-1 $\mathbf{H}$-indole (88) $\mathbf{8 8}$ was synthesized similarly to $\mathbf{8 6}$, starting from 2-bromo-1-(4-fluorophenyl)ethanone (118). Yield $42 \%$, mp $182-187^{\circ} \mathrm{C}$ (from toluene). Lit. ${ }^{44} \mathrm{mp} 185^{\circ} \mathrm{C}$.

2-(2-Methoxyphenyl)-1 $\mathbf{H}$-indole (89)—89 was synthesized similarly to $\mathbf{8 6}$, starting from 2-bromo-1-(2-methoxyphenyl)ethanone ${ }^{28}$ (119). Yield $41 \%, \mathrm{mp} 75-80^{\circ} \mathrm{C}$ (from toluene). Lit. ${ }^{45} \mathrm{mp} 78.5-81.0^{\circ} \mathrm{C}$.

2-(3-Methoxyphenyl)-1 $\mathrm{H}$-indole (90)—90 was synthesized similarly to $\mathbf{8 6}$, starting from 2-bromo-1-(3-methoxyphenyl)ethanone ${ }^{28}$ (120). Yield 40\%, mp 138-141 ${ }^{\circ} \mathrm{C}$ (from toluene). Lit. ${ }^{37} \mathrm{mp} 139.4-140.7^{\circ} \mathrm{C}$.

2-(3-Isopropoxy-4-methoxyphenyl)-1H-indole (101) - 101 was synthesized similarly to 86, starting from 2-bromo-1-(4-fluorophenyl)-ethanone (121). Yield 12\%, mp 137-141 ${ }^{\circ} \mathrm{C}$ (from ethanol). ${ }^{1} \mathrm{H}$ NMR $\left(\mathrm{CDCl}_{3}\right): \delta 1.42(\mathrm{~s}, 3 \mathrm{H}), 1.43(\mathrm{~s}, 3 \mathrm{H}), 3.90(\mathrm{~s}, 3 \mathrm{H}), 4.64(\mathrm{~m}$, $1 \mathrm{H}), 6.72(\mathrm{~s}, 1 \mathrm{H}), 6.95(\mathrm{~d}, J=8.2 \mathrm{~Hz}, 1 \mathrm{H}), 7.10-7.27(\mathrm{~m}, 5 \mathrm{H}), 7.39(\mathrm{~d}, J=8.0 \mathrm{~Hz}, 1 \mathrm{H}), 7.62$ $(\mathrm{d}, J=8.0 \mathrm{~Hz}, 1 \mathrm{H}), 8.3 \mathrm{ppm}$ (broad s, disappeared on treatment with $\mathrm{D}_{2} \mathrm{O}, 1 \mathrm{H}$ ). IR: $v 3366$ $\mathrm{cm}^{-1}$.

2-(Naphthalen-1-yl)-1 H-indole (102)—102 was synthesized similarly to 86, starting from 2-bromo-1-(naphthalen-1-yl)ethanone (122). Yield 28\%, mp 95-97 ${ }^{\circ} \mathrm{C}$ (from petroleum ether). Lit. $^{46} \mathrm{mp} 97-99^{\circ} \mathrm{C}$.

\section{General Procedure for the Preparation of Compounds 95-97, 100, and 104. Example: 2-( $p$ - Tolyl)-1H-indole (100)}

$n$-Butyllithium ( $4.2 \mathrm{~mL}, 0.0067 \mathrm{~mol}, 1.6 \mathrm{M}$ in $n$-hexane) was added dropwise to a $-40{ }^{\circ} \mathrm{C}$ solution of 4-methyl- $N$ - $(o$-tolyl)benzamide (123) $(0.5 \mathrm{~g}, 0.0022 \mathrm{~mol})$ in anhydrous THF (18 $\mathrm{mL}$ ). After being stirred at $-40^{\circ} \mathrm{C}$ for $1 \mathrm{~h}$, the reaction mixture was warmed at $0{ }^{\circ} \mathrm{C}$ for $1 \mathrm{~h}$ and then kept at $25^{\circ} \mathrm{C}$ for $12 \mathrm{~h}$. The mixture was diluted with water and extracted with ethyl acetate. The organic layer was washed with brine, dried, and filtered. Removal of the solvent gave a residue that was purified by column chromatography (silica gel, ethyl acetate/nhexane $=1: 5$ as eluent $)$ to furnish $100(0.2 \mathrm{~g}, 43 \%), \mathrm{mp} 217-219^{\circ} \mathrm{C}$ (from ethanol). Lit. ${ }^{45}$ mp $218.0-219.9^{\circ} \mathrm{C}$.

2-((1,1'-Biphenyl)-4-yl)-1 H-indole (104)-104 was synthesized similarly to $\mathbf{1 0 0}$, starting from $N$-(o-tolyl)-(1,1'-biphenyl)-4-carboxamide (124). Yield $22 \%, \mathrm{mp} 296-298{ }^{\circ} \mathrm{C}$ (from ethanol). ${ }^{1} \mathrm{H}$ NMR (DMSO- $\left.d_{6}\right): \delta 6.97-7.04(\mathrm{~m}, 2 \mathrm{H}), 7.10-7.14(\mathrm{t}, J=8.0 \mathrm{~Hz}, 1 \mathrm{H})$, 7.37-7.41 (m, 2H), 7.44-7.56 (m, 3H), 7.75-7.80 (m, 4H), 7.97 (d, J=8.0 Hz, 2H), 11,61 ppm (broad s, disappeared on treatment with $\mathrm{D}_{2} \mathrm{O}, 1 \mathrm{H}$ ). IR: $v 3681 \mathrm{~cm}^{-1}$. Lit. ${ }^{47} \mathrm{mp} 297-$ $298^{\circ} \mathrm{C}$.

2-Cyclobutyl-1 $\mathrm{H}$-indole (95) —95 was synthesized similarly to $\mathbf{1 0 0}$, starting from $\mathrm{N}$-(otolyl)cyclobutanecarboxamide (125). Yield $40 \%, \mathrm{mp} 73-76^{\circ} \mathrm{C}$ (from toluene). ${ }^{1} \mathrm{H}$ NMR 
(DMSO- $\left.d_{6}\right): \delta 1.86-2.03(\mathrm{~m}, 2 \mathrm{H}), 2.19-2.33(\mathrm{~m}, 2 \mathrm{H}), 2.58-2.54(\mathrm{~m}, 2 \mathrm{H}), 3.57-3.66(\mathrm{~m}$, 1H), 6.16-6.17 (m, 1H), 6.89-6.93 (m, 1H), 6.98-7.01 (m, 1H), 7.26-7.29 (m, 1H), 7.39$7.41(\mathrm{~m}, 1 \mathrm{H}), 10.90$ (broad s, disappeared on treatment with $\mathrm{D}_{2} \mathrm{O}, 1 \mathrm{H}$ ). IR: $v 3334 \mathrm{~cm}^{-1}$.

2-Cyclopentyl-1 $\mathrm{H}$-indole (96)—96 was synthesized similarly to 100 , starting from $\mathrm{N}$-(otolyl)cyclopentanecarboxamide (126). Yield $40 \%, \mathrm{mp} 80-82{ }^{\circ} \mathrm{C}$ (from toluene). Lit. ${ }^{48} \mathrm{mp}$ $84-85^{\circ} \mathrm{C}$.

2-Cyclohexyl-1 $\mathrm{H}$-indole (97)—97 was synthesized similarly to 100 , starting from $\mathrm{N}-(\mathrm{o}-$ tolyl)cyclohexanecarboxamide (127). Yield 59\%, mp 101-103 ${ }^{\circ} \mathrm{C}$ (from toluene). Lit. ${ }^{49} \mathrm{mp}$ $103-105^{\circ} \mathrm{C}$.

2-(1 H-Pyrazol-4-yl)-1 H-indole (98)—A mixture of $\mathbf{1 0 6}$ (0.1 g, $0.00041 \mathrm{~mol}), 1$-Bocpyrazole-4-boronic acid pinacol ester $(0.18 \mathrm{~g}, 0.000615 \mathrm{~mol})$, and $1 \mathrm{M}$ potassium carbonate $(4.1 \mathrm{~mL})$ in DMF $(2 \mathrm{~mL})$ was degassed for $30 \mathrm{~min} . \mathrm{PdCl}_{2}\left(\mathrm{PPh}_{3}\right)_{2}(0.003 \mathrm{~g}, 0.0041 \mathrm{mmol})$ was added, and the reaction mixture was placed into the microwave cavity (closed vessel mode, $P_{\max }=250 \mathrm{psi}$ ). A starting microwave irradiation of $250 \mathrm{~W}$ was used, the temperature being ramped from 25 to $160^{\circ} \mathrm{C}$, while stirring. Once $160{ }^{\circ} \mathrm{C}$ was reached, taking about $2 \mathrm{~min}$, the reaction mixture was held at this temperature for $10 \mathrm{~min}$. The reaction mixture was diluted with water, neutralized with $1 \mathrm{M} \mathrm{HCl}$, and extracted with ethyl acetate. The organic layer was washed with brine, dried, and filtered. Removal of the solvent gave a residue that was purified by column chromatography (silica gel, diethyl ether/ methanol $/ n$-hexane $=8: 1: 2$ as eluent) to furnish $79(0.02 \mathrm{~g}, 26 \%), \mathrm{mp} 220-225^{\circ} \mathrm{C}$ (from ethanol). ${ }^{1} \mathrm{H}$ NMR (DMSO- $\left.d_{6}\right): \delta 6.55(\mathrm{~s}, 1 \mathrm{H}), 6.97(\mathrm{t}, J=7.6 \mathrm{~Hz}, 1 \mathrm{H}), 7.00(\mathrm{t}, J=7.1 \mathrm{~Hz}$, $1 \mathrm{H}), 7.32(\mathrm{~d}, J=7.9 \mathrm{~Hz}, 1 \mathrm{H}), 7.44(\mathrm{~d}, J=7.7 \mathrm{~Hz}, 1 \mathrm{H}), 7.90-8.22(\mathrm{~m}, 2 \mathrm{H}), 11.24$ (broad s, disappeared on treatment with $\left.\mathrm{D}_{2} \mathrm{O}, 1 \mathrm{H}\right), 13.05 \mathrm{ppm}$ (broad s, disappeared on treatment with $\left.\mathrm{D}_{2} \mathrm{O}, 1 \mathrm{H}\right)$. IR: $v 2851,2921,3111,3400 \mathrm{~cm}^{-1}$.

3-((3,4,5-Trimethoxyphenyl)thio)-1 $H$-indole-2-carboxylic Acid (107)-107 was synthesized similarly to 14, starting from $\mathbf{1 0 5}$. Yield $45 \%, \mathrm{mp} 240-245^{\circ} \mathrm{C}$ (from ethanol). ${ }^{1} \mathrm{H}$ NMR (DMSO- $d_{6}$ ): $\delta 3.33$ (broad s, disappeared on treatment with $\mathrm{D}_{2} \mathrm{O}, 1 \mathrm{H}$ ), $3.56(\mathrm{~s}, 6 \mathrm{H}), 3.57(\mathrm{~s}, 3 \mathrm{H}), 6.42(\mathrm{~s}, 2 \mathrm{H}), 7.06-7.10(\mathrm{~m}, 1 \mathrm{H}), 7.26-7.30(\mathrm{~m}, 1 \mathrm{H}), 7.40-7.42$ $(\mathrm{m}, 1 \mathrm{H}), 7.47-7.49(\mathrm{~m}, 1 \mathrm{H}), 12.18$ (broad s, disappeared on treatment with $\left.\mathrm{D}_{2} \mathrm{O}, 1 \mathrm{H}\right), 13.10$ ppm (broad s, disappeared on treatment with $\mathrm{D}_{2} \mathrm{O}, 1 \mathrm{H}$ ). IR: $v 1738,2574,3329 \mathrm{~cm}^{-1}$.

2-lodo-3-((3,4,5-trimethoxyphenyl)thio)-1 $\boldsymbol{H}$-indole (108)-108 was synthesized similarly to $\mathbf{1 4}$, starting from 106. Yield $93 \%, \mathrm{mp} 135-140{ }^{\circ} \mathrm{C}$ (from ethanol). ${ }^{1} \mathrm{H}$ NMR $\left(\mathrm{CDCl}_{3}\right): \delta 3.71(\mathrm{~s}, 6 \mathrm{H}), 3.79(\mathrm{~s}, 6 \mathrm{H}), 6.68(\mathrm{~s}, 2 \mathrm{H}), 7.13-7.22(\mathrm{~m}, 2 \mathrm{H}), 7.37-7.39(\mathrm{~m}, 1 \mathrm{H})$, $7.61-7.63(\mathrm{~m}, 1 \mathrm{H}), 8.65 \mathrm{ppm}$ (broad s, disappeared on treatment with $\mathrm{D}_{2} \mathrm{O}, 1 \mathrm{H}$ ). IR: $v 3331$ $\mathrm{cm}^{-1}$.

2-(1 H-Indol-2-yl)oxazole (114)-114 was synthesized similarly to 31, using 106 and 2(tributylstannyl)oxazole. ${ }^{50}$ Yield $26 \%, \mathrm{mp} 33-35^{\circ} \mathrm{C}$ (from toluene). ${ }^{1} \mathrm{H}$ NMR $\left(\mathrm{CDCl}_{3}\right): \delta$ 7.16-7.20 (m, 2H), 7.27-7.32 (m, 2H), 7.40-7.43 (m, 1H), $7.71(\mathrm{~d}, J=8.0 \mathrm{~Hz}, 1 \mathrm{H}), 7.77$ (s, $1 \mathrm{H}), 8.65$ (broad s, disappeared on treatment with $\mathrm{D}_{2} \mathrm{O}, 1 \mathrm{H}$ ), $10.05 \mathrm{ppm}$ (broad s, disappeared on treatment with $\left.\mathrm{D}_{2} \mathrm{O}, 1 \mathrm{H}\right)$. IR: $v 3271 \mathrm{~cm}^{-1}$.

\section{General Procedure for the Preparation of Compounds 116-122. Example: 2-Bromo-1-(2- fluorophenyl)ethanone (116)}

A solution of bromine $(3.47 \mathrm{~g}, 1.1 \mathrm{~mL}, 0.0217 \mathrm{~mol})$ in anhydrous dichloromethane $(7 \mathrm{~mL})$ was dropped into a solution of 1-(2-fluorophenyl)-ethanone ( $3.0 \mathrm{~g}, 2.6 \mathrm{~mL}, 0.00217 \mathrm{~mol})$ in the same solvent $(14 \mathrm{~mL})$. The reaction mixture was stirred at $25^{\circ} \mathrm{C}$ for $2 \mathrm{~h}$, then diluted 
with a saturated aqueous solution of sodium hydrogen carbonate and extracted with dichloromethane. The organic layer was washed with brine, dried, and filtered. Removal of the solvent gave a residue that was purified by column chromatography (silica gel, dichloromethane/petroleum ether $=7: 3$ as eluent $)$ to furnish 116 as a slurry $(0.9 \mathrm{~g}, 20 \%) .51$

2-Bromo-1-(3-fluorophenyl)ethanone (117)-117 was synthesized similarly to 116, starting from 1-(3-fluorophenyl)ethanone. Yield $21 \%$ as a slurry. ${ }^{1} \mathrm{H}$ NMR (DMSO- $d_{6}$ ): $\delta$ $4.97(\mathrm{~s}, 2 \mathrm{H}), 7.53-7.65(\mathrm{~m}, 2 \mathrm{H}), 7.79-7.81(\mathrm{~m}, 1 \mathrm{H}), 7.85-7.87 \mathrm{ppm}(\mathrm{m}, 1 \mathrm{H})$. IR: $v 1686$ $\mathrm{cm}^{-1}$.

2-Bromo-1-(4-fluorophenyl)ethanone (118)-118 was synthesized similarly to $\mathbf{1 1 6}$, starting from 1-(4-fluorophenyl)ethanone. Yield 73\%, mp 47-49 ${ }^{\circ} \mathrm{C}$ (from $n$-hexane). ${ }^{1} \mathrm{H}$ NMR (DMSO- $\left.d_{6}\right): \delta 4.93(\mathrm{~s}, 2 \mathrm{H}), 7.37-7.49(\mathrm{~m}, 2 \mathrm{H}), 8.03-8.12 \mathrm{ppm}(\mathrm{m}, 2 \mathrm{H})$. IR: $v 1694$ $\mathrm{cm}^{-1}$. Lit. ${ }^{52} \mathrm{mp} 48-49{ }^{\circ} \mathrm{C}$.

2-Bromo-1-(3-isopropoxy-4-methoxyphenyl)ethanone (121)-A solution of 1-(3hydroxy-4-methoxyphenyl)ethanone $(0.25 \mathrm{~g}, 0.0015 \mathrm{~mol})$ in anhydrous DMF $(5 \mathrm{~mL})$ was added to a mixture of 2-iodopropane $(0.28 \mathrm{~g}, 0.2 \mathrm{~mL}, 0.00165 \mathrm{~mol})$ and potassium carbonate $(0.415 \mathrm{~g}, 0.003 \mathrm{~mol})$ in the same solvent $(5 \mathrm{~mL})$. The mixture was heated at $50{ }^{\circ} \mathrm{C}$ for $3 \mathrm{~h}$. After cooling, the mixture was neutralized with $1 \mathrm{M} \mathrm{HCl}$ and extracted with ethyl acetate. The organic layer was washed with brine, dried, and filtered. Removal of the solvent gave a residue that was purified by column chromatography (silica gel, ethyl acetate/ $n$ hexane $=3: 7$ as eluent $)$ to furnish 1-(3-isopropoxy-4-methoxyphenyl)-ethanone $(0.27 \mathrm{~g}$, $86 \%$ ), mp $53-56{ }^{\circ} \mathrm{C}$ (from toluene/ $n$-hexane). $\mathrm{Lit}^{53} \mathrm{mp} 56^{\circ} \mathrm{C}$. As described above for 116, 1-(3-isopropoxy-4-methoxyphenyl)ethanone was converted to 121, yield $65 \%$, mp $75-80^{\circ} \mathrm{C}$ (from ethanol).

Bromo-1-(naphthalen-1-yl)ethanone (122)—The compound was synthesized similarly to 116, starting from 1-(naphthalen-1-yl)-ethanone. Yield 90\%, mp 175-177 ${ }^{\circ} \mathrm{C}$ (petroleum ether). Lit. ${ }^{54} \mathrm{mp} 177-179{ }^{\circ} \mathrm{C}$.

\section{General Procedure for the Preparation of Compounds 123-127. Example N-(o-Tolyl)-4- methylbenzamide (123)}

To an ice-cooled solution of $o$-toluidine $(5.0 \mathrm{~g}, 5 \mathrm{~mL}, 0.0047 \mathrm{~mol})$ and triethyl-amine in anhydrous THF $(160 \mathrm{~mL})$ was added dropwise $p$-tolyl chloride $(8.66 \mathrm{~g}, 7.4 \mathrm{~mL}, 0.056 \mathrm{~mol})$. The mixture was stirred at $25^{\circ} \mathrm{C}$ for $12 \mathrm{~h}$. The mixture was diluted with a saturated solution of sodium hydrogen carbonate and extracted with ethyl acetate. The organic layer was washed with brine, dried, and filtered. Removal of the solvent gave a residue that was triturated with diethyl ether to furnish $\mathbf{1 2 3}(8.05 \mathrm{~g}, 76 \%), \mathrm{mp} 118-120{ }^{\circ} \mathrm{C}$ (from ethanol). Lit. ${ }^{55} \mathrm{mp} 121^{\circ} \mathrm{C}$.

$\mathbf{N}$-(o-Tolyl)(1,1'-biphenyl)-4-carboxamide (124)—124 was synthesized similarly to 123, starting from $\left(1,1^{\prime}\right.$-biphenyl)-4-carbonyl chloride. Yield $40 \%, \mathrm{mp} 176-178{ }^{\circ} \mathrm{C}$ (from ethanol). ${ }^{1} \mathrm{H}$ NMR $\left(\mathrm{CDCl}_{3}\right): \delta 2.39(\mathrm{~s}, 3 \mathrm{H}), 7.14-7.18(\mathrm{~m}, 1 \mathrm{H}), 7.27-7.33(\mathrm{~m}, 2 \mathrm{H}), 7.42-$ $7.44(\mathrm{~m}, 1 \mathrm{H}), 7.45-7.54(\mathrm{~m}, 2 \mathrm{H}), 7.65-7.68(\mathrm{~m}, 2 \mathrm{H}), 7.65-7.77(\mathrm{~m}, 3 \mathrm{H}), 7.98-8.02(\mathrm{~m}, 3 \mathrm{H})$. IR: $v 1637-3280 \mathrm{~cm}^{-1}$. Lit. ${ }^{56} \mathrm{mp} 179.5-180{ }^{\circ} \mathrm{C}$.

$\mathbf{N}$-(o-Tolyl)cyclobutanecarboxamide (125)—125 was synthesized similarly to $\mathbf{1 2 3}$, starting from cyclobutanecarbonyl chloride. Yield $90 \%, \mathrm{mp} 106-110{ }^{\circ} \mathrm{C}$ (from toluene). ${ }^{1} \mathrm{H}$ NMR $\left(\mathrm{CDCl}_{3}\right): \delta 1.91-2.07(\mathrm{~m}, 2 \mathrm{H}), 2.23(\mathrm{~s}, 3 \mathrm{H}), 2.26-2.44(\mathrm{~m}, 4 \mathrm{H}), 3.16-3.24(\mathrm{~m}, 1 \mathrm{H})$, 6.86 (broad s, disappeared on treatment with $\left.\mathrm{D}_{2} \mathrm{O}, 1 \mathrm{H}\right), 7.03-7.07(\mathrm{~m}, 1 \mathrm{H}), 7.16-7.22(\mathrm{~m}$, 2H), $7.86 \mathrm{ppm}(\mathrm{d}, J=7.8 \mathrm{~Hz}, 1 \mathrm{H})$. IR: $v 1646,3256 \mathrm{~cm}^{-1}$. 
$\mathbf{N}$-(o-Tolyl)cyclopentanecarboxamide (126)-126 was synthesized similarly to $\mathbf{1 2 3}$, starting from cyclopentanecarbonyl chloride. Yield $86 \%$, mp $125-129^{\circ} \mathrm{C}$ (from toluene). ${ }^{1} \mathrm{H}$ NMR (DMSO- $\left.d_{6}\right): \delta 1.52-1.88(\mathrm{~m}, 8 \mathrm{H}), 2.18(\mathrm{~s}, 3 \mathrm{H}), 2.81-2.88(\mathrm{~m}, 1 \mathrm{H}), 7.05-7.08(\mathrm{~m}$, $1 \mathrm{H}), 7.13-7.16(\mathrm{~m}, 1 \mathrm{H}), 7.19(\mathrm{~d}, J=7.6 \mathrm{~Hz}, 1 \mathrm{H}), 7.33(\mathrm{~d}, J=7.6 \mathrm{~Hz}, 1 \mathrm{H}), 9.19 \mathrm{ppm}$ (broad s, disappeared on treatment with $\left.\mathrm{D}_{2} \mathrm{O}, 1 \mathrm{H}\right)$. IR: $v 1648,3268 \mathrm{~cm}^{-1}$.

$\mathbf{N}$-(o-Tolyl)cyclohexanecarboxamide (127) - $\mathbf{1 2 7}$ was synthesized similarly to $\mathbf{1 2 3}$, starting from cyclohexanecarbonyl chloride. Yield $85 \%, \mathrm{mp} 150-155{ }^{\circ} \mathrm{C}$ (from toluene). ${ }^{1} \mathrm{H}$ NMR (DMSO- $\left.d_{6}\right): \delta 1.18-1.47(\mathrm{~m}, 5 \mathrm{H}), 1.64-1.84(\mathrm{~m}, 5 \mathrm{H}), 2.17(\mathrm{~s}, 3 \mathrm{H}), 2.37-2.42(\mathrm{~m}$, $1 \mathrm{H}), 7.05-7.08(\mathrm{~m} \mathrm{1H}), 7.12-7.16(\mathrm{~m}, 1 \mathrm{H}), 7.19(\mathrm{~d}, J=7.4 \mathrm{~Hz}, 1 \mathrm{H}), 7.32(\mathrm{~d}, J=7.1 \mathrm{~Hz}$, 1H), $9.13 \mathrm{ppm}$ (broad s, disappeared on treatment with $\mathrm{D}_{2} \mathrm{O}, 1 \mathrm{H}$ ). IR: $v 1649,3284 \mathrm{~cm}^{-1}$.

\section{General Procedure for the Preparation of Compounds 128 and 129. Example: 1-(1-(4- Chlorophenyl)ethylidene)-2-phenyl-hydrazine (128)}

A mixture of phenylhydrazine hydrochloride $(2.2 \mathrm{~g}, 0.015 \mathrm{~mol}), 4^{\prime}$-chloroacetophenone $(1.054 \mathrm{~g}, 1.3 \mathrm{~mL}, 0.01 \mathrm{~mol})$, and sodium acetate $(1.23 \mathrm{~g}, 0.015 \mathrm{~mol})$ in ethanol $(25 \mathrm{~mL})$ was placed into the microwave cavity (open vessel mode). Microwave irradiation of $250 \mathrm{~W}$ was used, the temperature being ramped from 25 to $80^{\circ} \mathrm{C}$. Once $80^{\circ} \mathrm{C}$ was reached, taking about $1 \mathrm{~min}$, the reaction mixture was held at this temperature for $5 \mathrm{~min}$ with stirring. The reaction mixture was cooled to $0{ }^{\circ} \mathrm{C}$, filtered, washed with petroleum ether, and dried to give $\mathbf{1 2 8}$ (3.38 g, 92\%), mp $114-116{ }^{\circ} \mathrm{C}$ (from ethanol). ${ }^{1} \mathrm{H}$ NMR $\left(\mathrm{CDCl}_{3}\right): \delta 2.23(\mathrm{~s}, 3 \mathrm{H}), 6.91$ (broad s, disappeared on treatment with $\left.\mathrm{D}_{2} \mathrm{O}, 1 \mathrm{H}\right), 7.17-7.19(\mathrm{~m}, 2 \mathrm{H}), 7.28-7.37(\mathrm{~m}, 5 \mathrm{H})$, 7.28-7.75 ppm (m, 2H). IR: $v 3349 \mathrm{~cm}^{-1}$. Lit. ${ }^{57} \mathrm{mp} 112-113{ }^{\circ} \mathrm{C}$.

1-(1-(4-Methoxyphenyl)ethylidene)-2-phenylhydrazine (129)-129 was synthesized similarly to 128, starting from $4^{\prime}$-methoxyacetophenone (166). Yield $95 \%, \mathrm{mp} 102-104{ }^{\circ} \mathrm{C}$ (from ethanol). ${ }^{1} \mathrm{H}$ NMR $\left(\mathrm{CDCl}_{3}\right): \delta 2.23(\mathrm{~s}, 3 \mathrm{H}), 3.85(\mathrm{~s}, 3 \mathrm{H}), 6.87$ (broad s, disappeared on treatment with $\left.\mathrm{D}_{2} \mathrm{O}, 1 \mathrm{H}\right), 6.90-6.94(\mathrm{~m}, 2 \mathrm{H}), 7.17-7.19(\mathrm{~m}, 2 \mathrm{H}), 7.28-7.31(\mathrm{~m}, 2 \mathrm{H})$, 7.75-7.77 ppm (m, 2H). IR: $v 3336 \mathrm{~cm}^{-1}$. Lit. ${ }^{58} \mathrm{mp} 97-98^{\circ} \mathrm{C}$.

\section{Biology. Tubulin Assembly}

The reaction mixtures contained $0.8 \mathrm{M}$ monosodium glutamate $(\mathrm{pH} 6.6$ with $\mathrm{HCl}$ in a $2 \mathrm{M}$ stock solution), $10 \mu \mathrm{M}$ tubulin, and varying concentrations of drug. Following a $15 \mathrm{~min}$ preincubation at $30^{\circ} \mathrm{C}$, samples were chilled on ice, GTP to $0.4 \mathrm{mM}$ was added, and turbidity development was followed at $350 \mathrm{~nm}$ in a temperature controlled recording spectrophotometer for $20 \mathrm{~min}$ at $30^{\circ} \mathrm{C}$. Extent of reaction was measured. Full experimental details were previously reported..$^{59}$

\section{$\left[{ }^{3} \mathrm{H}\right]$ Colchicine Binding Assay}

The reaction mixtures contained $1.0 \mu \mathrm{M}$ tubulin, $5.0 \mu \mathrm{M}\left[{ }^{3} \mathrm{H}\right]$ colchicine, and $5.0 \mu \mathrm{M}$ inhibitor and were incubated $10 \mathrm{~min}$ at $37^{\circ} \mathrm{C}$. Complete details were described previously. ${ }^{60}$

\section{Cell Cultures and Treatment}

Cell lines were obtained from the American Tissue Culture Collection (ATCC), unless specified otherwise. Cells were grown in Dulbecco's modified Eagle medium (DMEM) supplemented with $10 \%$ fetal bovine serum (FBS) at $37{ }^{\circ} \mathrm{C}$ with $5 \% \mathrm{CO}_{2}$. In all experiments 300000 cells were plated in $9 \mathrm{~cm}^{2}$ dishes and exposed to test compound dissolved in DMSO ( $0.1 \%$ final concentration) at the indicated concentrations.

HeLa, HT29, and A549 cell lines were grown at $37^{\circ} \mathrm{C}$ in DMEM containing $10 \mathrm{mM}$ glucose supplemented with $10 \% \mathrm{FBS}, 100 \mathrm{units} / \mathrm{mL}$ each of penicillin and streptomycin, and $2 \mathrm{mM}$ 
glutamine. At the onset of each experiment, cells were placed in fresh medium and cultured in the presence of test compounds from 0.01 to $25 \mu \mathrm{M}$.

231-MDA and A549 cells were cultured in DMEM supplemented with 10\% FBS for 24, 48, and $72 \mathrm{~h}$ in a 96 -well tissue culture plate at $37{ }^{\circ} \mathrm{C}$ and $5 \% \mathrm{CO}_{2}$ in the absence or presence of different drug concentrations.

HCT 116, HCT 15, Messa, and Messa/Dx5 were seeded into 96-well plates (Corning Inc., Costar) at a density of $2 \times 10^{3}$ cells/well in a volume of $50 \mu \mathrm{L}$ of the appropriate tissue culture medium. Compounds were added with different concentrations for the indicated incubation time at $37^{\circ} \mathrm{C}$ with $5 \% \mathrm{CO}_{2}$.

The human glioblastoma multiforme (GBM) cell line U87MG was purchased from the National Institute for Cancer Research (ICLC) of Genoa (Italy). The U87MG cells $\left(1 \times 10^{6}\right)$ were seeded onto T-75 flasks and cultured in RPMI medium supplemented with 10\% FBS, 2 $\mathrm{mM}_{\mathrm{L}}$-glutamine, $100 \mathrm{U} / \mathrm{mL}$ penicillin, $100 \mathrm{mg} / \mathrm{mL}$ streptomycin, and $1 \%$ nonessential amino acids at $37{ }^{\circ} \mathrm{C}$ in humidified atmosphere composed of oxygen (95\%) and carbon dioxide (5\%). For U87MG cell treatments, the cells were seeded at appropriate densities (5000 cells/well in 96-well plates or 50000 cells/well in 24-well plates) in complete medium. After 12 or $24 \mathrm{~h}$ the medium was replaced either with complete culture medium containing DMSO (untreated control cells) or with complete medium supplemented with the compound $\mathbf{1 8}$ or $\mathbf{5 7}$ dissolved in DMSO ( $<1 \% \mathrm{v} / \mathrm{v}$ of medium).

\section{Cell Viability Assay}

The methodology for the evaluation of the growth of human MCF-7 breast carcinoma, OVCAR-8, and NCI/ADR-RES cells, obtained from the National Cancer Institute drug screening laboratory, was previously described except that cells were grown for $96 \mathrm{~h}$ for $\mathrm{IC}_{50}$ determinations. ${ }^{61}$

Cell viability in Hela, HT-29, and A549 cells was determined using the 3-(4,5dimethylthiazol-2-yl)-2,5-diphenyltetrazolium bromide (MTT) colorimetric assay. The test is based on the ability of mitochondrial dehydrogenase to convert, in viable cells, the yellow MTT reagent into a soluble blue formazan dye. Cells were seeded into 96-well plates to a density of $7 \times 10^{3} / 100 \mu \mathrm{L}$ well. After $24 \mathrm{~h}$ of growth to allow attachment of cells to the wells, compounds were added at various concentrations (from 0.01 to $25 \mu \mathrm{M}$ ). After $48 \mathrm{~h}$ of growth and after removal of the culture medium, $100 \mu \mathrm{L} /$ well medium containing $1 \mathrm{mg} / \mathrm{mL}$ MTT was added. Cell cultures were further incubated at $37^{\circ} \mathrm{C}$ for $2 \mathrm{~h}$ in the dark. The solution was then gently aspirated from each well, and the formazan crystals within the cells were dissolved with $100 \mu \mathrm{L}$ of DMSO. Optical densities were read at $550 \mathrm{~nm}$ using a Multiskan Spectrum Thermo Electron Corporation reader. Results were expressed as a percentage relative to vehicle-treated control (0.5\% DMSO was added to untreated cells), and the $\mathrm{IC}_{50}$ values were determined by linear and polynomial regression. Experiments were performed in triplicate.

231-MDA and A549 cells were plated at different cellular densities in order to test the compounds on logarithmic phase cells. Test compounds were added after cell adhesion. On the day of the assay, $10 \mu \mathrm{L}$ of MTT $(5 \mathrm{mg} / \mathrm{mL}$ ) was added to each well, and cells were incubated for $2 \mathrm{~h}\left(37^{\circ} \mathrm{C}, 5 \% \mathrm{CO}_{2}\right)$. When dark crystals appeared at the well bottom, culture medium was discarded, and ethanol $(100 \mu \mathrm{L})$ was added to each well to solubilize the crystals, yielding a purple solution. Absorbance was read with an enzyme-linked immunosorbent assay (ELISA) reader at $570 \mathrm{~nm}$. 
The antiproliferative effect of the tubulin polymerization inhibitors on cell proliferation was evaluated against HCT 116, HCT 15, Messa, and Messa/Dx5 tumor cell lines using the CellTiter-Glo luminescent cell viability assay (Promega, Madison, WI) according to the manufacturer's instructions. The cells, in exponential growth, were incubated for $72 \mathrm{~h}$ at different concentrations of the inhibitors. Then an equivalent of the CellTiter-Glo reagent was added, the solution was mixed for $2 \mathrm{~min}$ in order to induce cell lysis, and the luminescence was recorded after a further $10 \mathrm{~min}$. $\mathrm{GI}_{50}$ values were calculated using the parameters of a nonlinear regression analysis program (GraphPad Prism statistics software).

The effects of the treatments with $\mathbf{1 8}$ or $\mathbf{5 7}$ on U87MG cell survival/growth were estimated by a colorimetric MTS conversion assay ${ }^{32}$ and trypan blue exclusion assay. ${ }^{59}$ The MTS assay was used to determine $\mathrm{IC}_{50}$ values on $\mathrm{U} 87 \mathrm{MG}$ cell survival following $24 \mathrm{~h}$ of treatment. U87MG cells were seeded in 96-well plates and incubated with compound concentrations ranging from $0.1 \mathrm{nM}$ to $20 \mu \mathrm{M}$ (DMSO as control). After $24 \mathrm{~h}$, MTS reagent was added, and the absorbance at $590 \mathrm{~nm}$ was measured by a microplate reader (Wallac, Victor 2, 1420 multilabel counter, PerkinElmer). The percentage of proliferating cells after compound exposure was calculated with respect to control cells (100\%). Sigmoid dose response curves were performed using GraphPad Prism 4 software (GraphPad Software Inc., San Diego, CA).

The trypan blue exclusion assay was used to examine the effects of compound treatments on U87MG cell survival. U87MG cells were seeded in 24-well plates and exposed to $1 \mathrm{nM}$ to $10 \mu \mathrm{M}$ compound concentrations for 8,24 , and $48 \mathrm{~h}$. After exposure, the number of viable (white) and dead (blue) cells was counted using a microscope, and the percentage of viable or dead cells was calculated with respect to control cells (100\%).

\section{Immunofluorescence (IF) and Microscopy}

HeLa epithelial cells were grown in Dulbecco's modified Eagle medium (DMEM) supplemented with $10 \% \mathrm{FBS}$ at $37^{\circ} \mathrm{C}$ with $5 \% \mathrm{CO}_{2}$. All compounds were dissolved in DMSO ( $0.1 \%$ final concentration) and used at the indicated concentrations for $24 \mathrm{~h}$.

HeLa cells were seeded at $3 \times 10^{5}$ cells in culture dishes containing sterile coverslips coated with poly-L-lysine. At the end of the treatment, they were directly fixed in methanol for 10 min at $-20{ }^{\circ} \mathrm{C}$ and processed for IF with either mouse anti- $\boldsymbol{a}$-tubulin antibody (Sigma clone B5.1.2, 1:2000 dilution) followed by FITC-conjugated goat anti-mouse secondary antibody (Jackson Immunoresearch Laboratories, 115-095-068, 1:200) or with mouse anti-cyclin B1 antibody (Santa Cruz, GNS1, 1:50) followed by Texas Red conjugated horse anti-mouse (Vector, TI-2000, 1:800) secondary antibody. Slides were counterstained with $0.05 \mu \mathrm{g} / \mathrm{mL}$ DAPI (Sigma) and mounted in Vectashield (Vector). Cells were analyzed under an epifluorescence Olympus AX70 microscope with a CCD camera (Diagnostic Instrument, Spot RT slider model 2.3.0). Unfixed cell cultures were observed under an inverted NIKON TE 300 microscope with a 10x objective, and images were acquired using the ACT-1 software and a DMX1200 CCD (resolution $1280 \times 1024$ pixels).

\section{Flow Cytometric Analysis}

Cell cycle distribution was analyzed after incubation with PI (Sigma-Aldrich), whereas apoptosis was analyzed with annexin V-FITC (Immunological Sciences, IK-11120) staining alone or with annexin V-FITC in combination with PI. Cell samples were analyzed in a Coulter Epics XL cytofluorometer (Beckman Coulter) equipped with EXPO 32 ADC software. Data from at least 10000 cells per sample were acquired. 


\section{Evaluation of Mitochondrial Potential}

JC-1 (Invitrogen, T3168, Carlsbad, CA) is a cationic dye that exhibits potential-dependent accumulation in mitochondria indicated by a fluorescence emission shift from green ( 525 $\mathrm{nm})$ to red $(\sim 590 \mathrm{~nm})$. Consequently, mitochondrial depolarization is indicated by a decrease in the red/green fluorescence intensity ratio and can be quantified by using fluorescence microscopy. ${ }^{33 \mathrm{~b}}$ To evaluate the mitochondrial depolarization induced by drug treatment, U87MG cells were seeded in 96-well plates and stained for $10 \mathrm{~min}$ in medium containing JC- 1 at a final concentration of $50 \mu \mathrm{g} / \mathrm{mL}$. After removal of JC-1, fresh medium containing the drug at $100 \mathrm{nM}$ (approximately its $\mathrm{IC}_{50}$ value in U87MG cells) or $30 \mu \mathrm{M}$ $\mathrm{CCCP}$, a standard mitochondrial potential dissipation drug, was added to the cells. Immediately and $24 \mathrm{~h}$ after the treatment, pictures were taken under an Axiovert fluorescent microscope (Zeiss) using the filter set 10, 488010-0000 (Zeiss) (excitation 450-490 nm, emission 515-565 nm). Pictures were then split in the RGB channels (red and green) and analyzed by using the program ImageJ. ${ }^{62}$ For each treatment, the red/green fluorescence ratio obtained at $24 \mathrm{~h}$ was normalized with respect to red/green fluorescence ratio obtained at $0 \mathrm{~h}$.

\section{ROS Production}

The generation of ROS was assessed by the fluorogenic probe DCFH2-DA (Molecular Probes, Invitrogen) in U87MG cells after compound exposure (DMSO, $\mathrm{H}_{2} \mathrm{O}_{2}$ as control standard). DCFH2-DA is a reduced and acetylated form of fluorescein used as an indicator for ROS in cells. ${ }^{63}$ This nonfluorescent molecule is readily converted to a green-fluorescent form (FDA) when the acetate group is removed by intracellular esterases and an oxidation by ROS occurs within the cell.

U87MG cells were seeded in 96-wells plate, washed in PBS/10 mM glucose, and loaded with $10 \mu \mathrm{M}$ DCFH2-DA for $30 \mathrm{~min}$ in the dark at $37^{\circ} \mathrm{C}$. Afterward, the cells were washed with the same medium and incubated with or without the compound at $100 \mathrm{nM}$.

Fluorescence increase was estimated in a plate reader at $485 \mathrm{~nm}$ (excitation) and $520 \mathrm{~nm}$ (emission) after 1, 5, 15, and $60 \mathrm{~min}$ (Wallac, Victor 2, 1420 multilabel counter, PerkinElmer). The fluorescence values were normalized between samples for cell number content and assessed by a crystal violet cell staining assay. Crystal violet is an intense stain that binds to cell nuclei and gives an $A_{595}$ reading that is proportional to cell number. After washing with PBS/10 mM glucose, the cells were incubated with crystal violet for $30 \mathrm{~min}$ at room temperature. After washing, a solution of $1 \%$ SDS was added to each well, and the plates were shaken mechanically for $1 \mathrm{~h}$ at room temperature. $A_{595}$ was then determined on a plate reader. The measured optical density readings indicated the relative number of cells in each well, and this was used to normalize the absorbance obtained with FDA.

\section{Statistical Analyses}

For data analysis and graphic presentations, the nonlinear multipurpose curve-fitting program GraphPad Prism (GraphPad) was used. All data are presented as the mean \pm SEM. Statistical analysis was performed by one-way analysis of variance (ANOVA) with Bonferroni's corrected $t$ test for posthoc pairwise comparisons. $P<0.05$ was considered statistically significant.

\section{In Vivo Vascular Disrupting Effects}

This study was approved by the institutional ethical committee for the use and care of laboratory animals. Six adult WAG/Rij rats (Iffa Credo, Brussels, Belgium) weighing $250 \mathrm{~g}$ received intrahepatic implantation of bifocal rhabdomyosarcoma allografts, which mimics hypervascular human liver metastases as previously described. ${ }^{34}$ Three rats bearing six liver 
tumors in total were thus prepared for studying $\mathbf{1 8}$ and $\mathbf{5 7}$ separately. The growth of tumors was monitored with MRI until their diameter reached about $10 \mathrm{~mm}$ for therapy. MRI was carried out with a clinical 3.0 T whole-body MR magnet (Trio; Siemens, Erlangen, Germany) with a maximum gradient capability of $45 \mathrm{mT} / \mathrm{m}$ by using an eight-channel phased-array wrist coil (In Vivo, Latham, NY). The rat was gas-anesthetized with 2\% isoflurane in a mixture of $20 \%$ oxygen and $80 \%$ room air, through a mask connected by a tube to a Harvard Apparatus system (Holliston, MA) and placed supine in a plastic holder. The penile vein of the rat was cannulated for MRI contrast agent or VDA compound administration. With intravenous bolus of gadoterate meglumine (Dotarem; Guerbet, Brussels, Belgium) at $0.1 \mathrm{mmol} / \mathrm{kg}$, contrast enhanced $T_{1}$ weighted MRI (CE-T1WI) ${ }^{35}$ was performed at baseline at 1 and $24 \mathrm{~h}$ post-VDA treatment to document intratumoral vascular disrupting event. After baseline CE-T1WI, a VDA solution of $\mathbf{1 8}$ or $\mathbf{5 7}$ was intravenously injected at a dose of $15 \mathrm{mg} / \mathrm{kg}$ for subsequent evaluation of the therapeutic effects.

\section{LC-MS/MS Analytical Method}

Samples from the metabolic stability, solubility, and permeability assays were analyzed under the following conditions: UPLC instrument (Waters) interfaced with a Premiere XE triple quadrupole instrument (Waters). Eluents were the following: phase A consisting of $95 \% \mathrm{H}_{2} \mathrm{O}, 5 \%$ acetonitrile $+0.1 \% \mathrm{HCOOH}$; phase B consisting of $5 \% \mathrm{H}_{2} \mathrm{O}, 95 \%$ acetonitrile $+0.1 \% \mathrm{HCOOH}$. Parameters were as follows: flow rate, $0.6 \mathrm{~mL} / \mathrm{min}$; column, Acquity BEH C18, $50 \mathrm{~mm} \times 2.1 \mathrm{~mm}, 1.7 \mu \mathrm{m}$ at $50{ }^{\circ} \mathrm{C}$; injection volume, $5 \mu \mathrm{L}$. LC-MS/MS analyses were carried out using an ESI(+) interface in multiple reaction monitoring mode.

\section{Metabolic Stability}

Compounds 18, 20, 55, and $\mathbf{5 7}$ were dissolved in DMSO in duplicate at a final concentration of $1 \mu \mathrm{M}$ and preincubated for $10 \mathrm{~min}$ at $37^{\circ} \mathrm{C}$ in potassium phosphate buffer, $\mathrm{pH} 7.4,3 \mathrm{mM}$ $\mathrm{MgCl}_{2}$, with rat liver microsomes (Xenotech) at a final concentration of $0.5 \mathrm{mg} / \mathrm{mL}$. After the preincubation period, reactions were started by adding the cofactors mixture (NADP, Glc6P, G6P-DH). Samples were taken at times 0 and $30 \mathrm{~min}$ and added to acetonitrile to stop the reaction and centrifuged. Supernatants were analyzed and quantified by LC-MS/ MS. A control sample without cofactors was always added in order to check the stability of test compounds in the reaction mixtures. 7-Ethoxycoumarin was used as a reference standard. A fixed concentration of verapamil was added in every sample as an internal standard for LC-MS/MS. The percent of test compound remaining after a 30 min incubation period was calculated from the peak area relative to the area of the compound after the 30 min incubation time.

\section{Aqueous Solubility}

The solubilities of compounds $\mathbf{1 8}, \mathbf{2 0}, \mathbf{5 5}$, and $\mathbf{5 7}$ were measured using a high throughput screening assay format. Samples prepared at the target concentration of $200 \mu \mathrm{M}$ were placed in a 96-well filter plate and incubated at room temperature for $90 \mathrm{~min}$. The plate was then filtered, and solutions were analyzed by LC/MS-UV. Final concentrations are evaluated by comparing the area under the curve of the $\mathrm{MeOH}$ stock solution with those of the test compound solutions.

\section{Caco-2 Cell Permeability}

Caco-2 cells (ECACC) were cultured in DMEM, 10\% FBS, 1\% NEAA, 10 mM Hepes buffer, $50 \mathrm{U}$ penicillin, and $50 \mathrm{ug} / \mathrm{mL}$ streptomycin and split at confluence by trypsinization. For transport studies, 200000 cells/well were seeded on Millicell 24-well cell culture plates. After $24 \mathrm{~h}$ at $37^{\circ} \mathrm{C}$ in a humidified, $5 \% \mathrm{CO}_{2}$ atmosphere, the medium was exchanged for enterocyte differentiation medium with additives (Becton Dickinson), which allows Caco-2 
cells to establish within 3 days a differentiated enterocyte monolayer. TEER, measured with a Millicell-ERS (Millipore, Corp), must be $>1000 \Omega$. Transport across the Caco-2 monolayer was determined by adding a $10 \mu \mathrm{M}$ solution of compound in DMEM (1\% final concentration of DMSO) to the side from which permeability was to be determined. The $\mathrm{A} \rightarrow \mathrm{B}$ transport across Caco-2 monolayer cells was determined by adding the compound to the apical side at $\mathrm{pH} 6.5$. After $2 \mathrm{~h}$, the basolateral side solution at $\mathrm{pH} 7.4$ and the apical and starting solutions were analyzed by LC-MS/MS. In the B $\rightarrow$ A experiment, the compound was added to the basolateral side and collected on the apical side. Monolayer integrity was assessed with a lucifer yellow assay at the end of each experiment. The apparent permeability $\left(P_{\text {app }}\right)$ was calculated with the following equation:

$$
P_{\text {app }}=J / C_{0}
$$

where $J$ is the flux (dX/dt per $A$ ) and $C_{0}$ is the donor concentration $(\mu \mathrm{M})$ at $t=0 ; \mathrm{d} X / \mathrm{d} t$ is the change in mass $(X, \mathrm{nmol})$ per time $(t, \mathrm{~s})$, and $A$ is the filter surface area $\left(\mathrm{cm}^{2}\right)$.

\section{CYP450 Inhibition}

Compounds 18, 20, 55, and $\mathbf{5 7}$ were evaluated in a 96-well plate containing an appropriate buffer and a NADPH regenerating system. Cytochrome P450 inhibition experiments were carried out according to the manufacturer's instructions (BD Biosciences, Franklin Lakes, NJ, U.S.). The compounds were dissolved in a 96-well plate at a $10 \mu \mathrm{M}$ final concentration in potassium phosphate buffer ( $\mathrm{pH}$ 7.4) containing an NADPH regenerating system. For all enzyme/substrate pairs, the final cofactor concentrations were $1.3 \mathrm{mM} \mathrm{NADP}{ }^{+}, 3.3 \mathrm{mM}$ glucose 6-phosphate, and $0.4 \mathrm{U} / \mathrm{mL}$ glucose 6-phosphate dehydrogenase. The reaction was initiated by the addition of specific isoenzymes (Supersomes, Gentest) and substrates at 37 ${ }^{\circ} \mathrm{C}$. Furafylline (for CYP1A2, $100 \mathrm{mM}$ ), sulfaphenazole (for CYP2C9, $10 \mathrm{mM}$ ), tranylcypromine (for CYP2C19, $500 \mu \mathrm{M}$ ), quinidine (for CYP2D6, $0.5 \mathrm{mM}$ ), and ketoconazole (for CYP3A4, $1.66 \mathrm{mM}$ ) were employed as control inhibitors in one-third serial dilution. Incubations were carried out for $15 \mathrm{~min}(0.5 \mathrm{pmol}$ of CYP1A2, $5 \mathrm{mM} 3-$ cyano-7-ethoxycoumarin), $30 \mathrm{~min}$ (0.5 pmol of CYP2C19, $25 \mathrm{mM}$ 3-cyano-7ethoxycoumarin, 1 pmol of CYP3A4, $50 \mathrm{mM}$ 7-benzyloxy-4-(trifluoromethyl)coumarin; 1.5 pmol of CYP2D6, $1.5 \mathrm{mM} 3$-[2-( $N, N$-diethyl- $N$-methylamino)-ethyl]-7-methoxy-4methylcoumarin), or $45 \mathrm{~min}$ (1 pmol of CYP2C9, $75 \mathrm{mM} 7$-methoxy-4-

(trifluoromethyl)coumarin). The reaction was then quenched by adding $75 \mathrm{~mL}$ of a mixture containing $80 \% \mathrm{CH}_{3} \mathrm{CN}$ and $20 \%$ Tris base $(0.5 \mathrm{~m})$, and plates were read on a fluorimeter at the appropriate emission/excitation wavelengths. The percentage inhibition was calculated relative to enzyme samples without inhibitors.

\section{Supplementary Material}

Refer to Web version on PubMed Central for supplementary material.

\section{Acknowledgments}

This research was supported by PRIN 2008 (Grant 200879X9N9), Progetti di Ricerca di Università, Sapienza Università di Roma (Grant 2011), and Bando Futuro in Ricerca 2010 (Grant RBFR10ZJQT). The authors also thank FILAS (Finanziaria Laziale di Sviluppo, Grant 2010) for partial support. A.C. thanks Istituto Pasteur-Fondazione Cenci Bolognetti for his Borsa di Studio per Ricerche in Italia. A.R. thanks Bando Futuro in Ricerca 2010 for her Borsa di Studio. We are grateful to Alessio Bolognesi, Maria Giubettini, and Annalisa Verrico for experimental work in cell cycle analysis. This work was supported in part by funds from AIRC (Grant IG 10164) to P.L. 


\section{ABBREVIATIONS USED}

MT

ATI

CSA4

VBL

PTX

VRB

SAR

$\boldsymbol{P}_{\max }$

BOP

$\mathbf{P d}_{2}(\mathrm{dba})_{3}$

$\mathbf{P}(\mathrm{Cy})$

$\mathrm{Pd}(\mathrm{dppf})-\mathrm{Cl}_{2} \cdot \mathrm{CH}_{2} \mathrm{Cl}_{2}$

$\mathbf{P d C l}_{2}\left(\mathbf{P P h}_{3}\right)_{2}$

$\operatorname{Pd}(\mathrm{OAc})_{2}$

$\mathbf{P d}\left(\mathbf{P P h}_{3}\right)_{4}$

SPhos

MI

DAPI

Pgp

FBS

CCCP

DCFH2-DA

ROS

JC-1

FDA microtubule

arylthioindole

combretastatin A-4

vinblastine

paclitaxel

vinorelbine

structure-activity relationship

maximum pressure

benzotriazol-1-yloxytris(dimethylamino)phosphonium hexafluorophosphate

tris(dibenzylideneacetone)-dipalladium(0)

tricyclohexylphosphine

1,1' 'bis(diphenylphosphino)ferrocene]-dichloropalladium(II), complex with dichloromethane

bis(triphenylphosphine)palladium(II) dichloride

palladium(II) acetate

tetrakis-(triphenylphosphine)palladium(0)

2-dicyclohexylphosphino-2', $6^{\prime}$-dimethoxybiphenyl

mitotic index

4',6-diamidino-2-phenylindole

P-glycoprotein

fetal bovine serum

carbonyl cyanide $m$-chlorophenylhydrazone

6-carboxy-2 ${ }^{\prime}, 7^{\prime}$-dichlorodihydrofluorescein diacetate

reactive oxygen species

$5,5^{\prime}, 6,6^{\prime}$-tetrachloro-1, $1^{\prime} 3,3^{\prime}$ -

tetraethylbenzimidazoylcarbocyanine iodide

green-fluorescent form of 6-carboxy- $2^{\prime}, 7^{\prime}$ -

dichlorodihydrofluorescein diacetate

vascular disrupting agent

\section{References}

1. Jordan MA, Wilson L. Microtubules as a target for anticancer drugs. Nat Rev Cancer. 2004; 4:253265. [PubMed: 15057285]

2. Honore S, Pasquier E, Braguer D. Undestanding microtubule dynamics for improved cancer therapy. Cell Mol Life Sci. 2005; 62:3039-3056. [PubMed: 16314924]

3. Hsieh HP, Liou JP, Mahindroo N. Pharmaceutical design of antimitotic agents based on combretastatins. Curr Pharm Des. 2005; 11:1655-1677. [PubMed: 15892667] 
4. Tron GC, Pirali T, Sorba G, Pagliai F, Busacca S, Genazzani AA. Medicinal chemistry of combretastatin A4: present and future directions. J Med Chem. 2006; 49:3033-3044. [PubMed: 16722619]

5. Teicher BA. Newer cytotoxic agents: attacking cancer broadly. Clin Cancer Res. 2008; 14:16101617. [PubMed: 18347161]

6. Bhattacharyya B, Panda D, Gupta S, Banerjee M. Anti-mitotic activity of colchicine and the structural basis for its interaction with tubulin. Med Res Rev. 2008; 28:155-183. [PubMed: 17464966]

7. Ravelli RB, Gigant B, Curmi PA, Jourdain I, Lachkar S, Sobel A, Knossow M. Insight into tubulin regulation from a complex with colchicine and a stathmin-like domain. Nature. 2004; 428:198-202. [PubMed: 15014504]

8. Lin MC, Ho HH, Pettit GR, Hamel E. Antimitotic natural products combretastatin A-4 and combretastatin A-2: studies on the mechanism of their inhibition of the binding to colchicine to tubulin. Biochemistry. 1989; 28:6984-6991. [PubMed: 2819042]

9. Nogales E, Whittaker M, Milligan RA, Downing KH. High-resolution model of the microtubule. Cell. 1999; 96:79-88. [PubMed: 9989499]

10. Nettles JH, Li H, Cornett B, Krahn JM, Snyder JP, Downing KH. The binding mode of epothilone A on alpha, beta-tubulin by electron crystallography. Science. 2004; 305:866-869. [PubMed: 15297674]

11. Buey RM, Calvo E, Barasoain I, Pineda O, Edler MC, Matesanz R, Cerezo G, Vanderwal CD, Day BW, Sorensen EJ, Lopez JA, Andreu JM, Hamel E, Diaz JF. Cyclostreptin binds covalently to microtubule pores and lumenal taxoid binding sites. Nat Chem Biol. 2007; 3:117-125. [PubMed: 17206139]

12. Beckers T, Mahboobi S. Natural, semisynthetic and synthetic microtubule inhibitors for cancer therapy. Drugs Future. 2003; 28:767-785.

13. Sridhare M, Macapinlac MJ, Goel S, Verdier-Pinard D, Fojo T, Rothenberg M, Colevas D. The clinical development of new mitotic inhibitors that stabilize the microtubule. Anti-Cancer Drugs. 2004; 15:553-555. [PubMed: 15205596]

14. Schmidt M, Bastians H. Mitotic drug targets and the development of novel anti-mitotic anticancer drugs. Drug Resist Updates. 2007; 10:162-181.

15. De Martino G, La Regina G, Coluccia A, Edler MC, Barbera MC, Brancale A, Wilcox E, Hamel E, Artico M, Silvestri R. Arylthioindoles, potent inhibitors of tubulin polymerization. J Med Chem. 2004; 47:6120-6123. [PubMed: 15566282]

16. De Martino G, Edler MC, La Regina G, Coluccia A, Barbera MC, Barrow D, Nicholson RI, Chiosis G, Brancale A, Hamel E, Artico M, Silvestri R. Arylthioindoles, potent inhibitors of tubulin polymerization. 2. Structure activity relationships and molecular modeling studies. J Med Chem. 2006; 49:947-954. [PubMed: 16451061]

17. La Regina G, Edler MC, Brancale A, Kandil S, Coluccia A, Piscitelli F, Hamel E, De Martino G, Matesanz R, Díaz JF, Scovassi AI, Prosperi E, Lavecchia A, Novellino E, Artico M, Silvestri R. New arythioindoles inhibitors of tubulin polymerization. 3. Biological evaluation, SAR and molecular modeling studies. J Med Chem. 2007; 50:2865-2874. [PubMed: 17497841]

18. La Regina G, Sarkar T, Bai R, Edler MC, Saletti R, Coluccia A, Piscitelli F, Minelli L, Gatti V, Mazzoccoli C, Palermo V, Mazzoni C, Falcone C, Scovassi AI, Giansanti V, Campiglia P, Porta A, Maresca B, Hamel E, Brancale A, Novellino E, Silvestri R. New arylthioindoles and related bioisosteres at the sulfur bridging group. 4. Synthesis, tubulin polymerization, cell growth inhibition, and molecular modeling studies. J Med Chem. 2009; 52:7512-7527. [PubMed: 19601594]

19. La Regina G, Bai R, Rensen W, Coluccia A, Piscitelli F, Gatti V, Bolognesi A, Lavecchia A, Granata I, Porta A, Maresca B, Soriani A, Iannitto ML, Mariani M, Santoni A, Brancale A, Ferlini C, Dondio G, Varasi M, Mercurio C, Hamel E, Lavia P, Novellino E, Silvestri R. Design and synthesis of 2-heterocyclyl-3-arylthio- $1 H$-indoles as potent tubulin polymerization and cell growth inhibitors with improved metabolic stability. J Med Chem. 2011; 54:8394-8406. [PubMed: 22044164] 
20. Vitale I, Galluzzi L, Castedo M, Kroemer G. Mitotic catastrophe: a mechanism for avoiding genomic instability. Nat Rev Mol Cell Biol. 2011; 12:385-392. [PubMed: 21527953]

21. Poirier M, Goudreau S, Poulin J, Savoie J, Beaulieu PL. Metal-free coupling of azoles with 2- and 3-haloindoles providing access to novel 2- or 3-(azol-1-yl)indole derivatives. Org Lett. 2010; 12:2334-2337. [PubMed: 20397735]

22. Gavina, BD.; Forte, B.; Mantegani, S.; Varasi, M.; Vianello, P. Preparation of Pyrazolyl-indole Derivatives Active As Kinase Inhibitors. PCT Int. Appl. WO 2005005414 A2. Jan 20. 2005

23. Bergman J, Venemalm L. Efficient synthesis of 2-chloro-, 2-bromo-, and 2-iodoindole. J Org Chem. 1992; 57:2495-2497.

24. Hudkins RL, Diebold JL, Marsh FD. Synthesis of 2-aryl- and 2-vinyl-1H-indoles via palladiumcatalyzed cross-coupling of aryl and vinyl halides with 1-carboxy-2-(tributylstannyl)indole. J Org Chem. 1995; 60:6218-6220.

25. Hugon B, Pfeiffer B, Renard P, Prudhomme M. Synthesis of isogranulatimide analogues possessing a pyrrole moiety instead of an imidazole heterocycle. Tetrahedron Lett. 2003; 44:39273930.

26. Kolhatkar RB, Ghorai SK, George C, Reith MEA, Dutta AK. Interaction of cis-(6benzhydrylpiperidin-3-yl)benzylamine analogues with monoamine transporters: structure-activity relationship study of structurally constrained 3,6-disubstituted piperidine analogues of (2,2diphenylethyl)-[1-(4-fluorobenzyl)piperidin-4-ylmethyl]amine. J Med Chem. 2003; 46:22052215. [PubMed: 12747792]

27. (a) Hoover, DJ.; Witter, KG. Heteroaromatic Compounds as PDE10a Inhibitors and Their Preparation, Pharmaceutical Compositions and Use in the Treatment of Central Nervous System Diseases. PCT Int Appl WO 2008004117. Jan 10. 2008 p. A1(b) Koradin C, Dohle W, Rodriguez AL, Schmid B, Knochel P. Synthesis of polyfunctional indoles and related heterocycles mediated by cesium and potassium bases. Tetrahedron. 2003; 59:1571-1587.

28. This compound was also purchased from Sigma-Aldrich.

29. La Regina G, Gatti V, Piscitelli F, Silvestri R. Open vessel and cooling while heating microwaveassisted synthesis of pyridinyl $N$-aryl hydrazones. ACS Comb Sci. 2011; 13:2-6. [PubMed: 21247117]

30. (a) Vakifahmetoglu HM, Olsson M, Zhivotovsky B. Death through a tragedy: mitotic catastrophe. Cell Death Differ. 2008; 15:1153-1162. [PubMed: 18404154] (b) Gascoigne KE, Taylor SS. How do anti-mitotic drugs kill cancer cells? J Cell Sci. 2009; 122:2579-2585. [PubMed: 19625502] (c) Vitale I, Galluzzi L, Castedo M, Kroemer G. Mitotic catastrophe: a mechanism for avoiding genomic instability. Nat Rev Mol Cell Biol. 2011; 12:385-392. [PubMed: 21527953]

31. Rieder C, Cole R. Microtubule disassembly delays the G2-M transition in vertebrates. Curr Biol. 2000; 10:1067-1070. [PubMed: 10996076]

32. (a) Mantymaa P, Siitonen T, Guttorm T, Saily M, Kinnula V, Savolainen ER, Koistinen P. Induction of mitochondrial manganese superoxide dismutase confers resistance to apoptosis in acute myeloblastic leukaemia cells exposed to etoposide. Br J Haematol. 2000; 108:574-581. [PubMed: 10759716] (b) Chelli B, Lena A, Vanacore R, Da Pozzo E, Costa B, Rossi L, Salvetti A, Scatena F, Ceruti S, Abbracchio MP, Gremigni V, Martini C. Peripheral benzodiazepine receptor ligands: mitochondrial transmembrane potential depolarization and apoptosis induction in rat C6 glioma cells. Biochem Pharmacol. 2004; 68:125-134. [PubMed: 15183124]

33. Nohl H, Gille L, Staniek K. Intracellular generation of reactive oxygen species by mitochondria. Biochem. Pharmacol. 2005; 69: 719-723. (b) Szilágyi, G., Simon, L., Koska, P., Telek, G., Nagy, $\mathrm{Z}$. Visualization of mitochondrial membrane potential and reactive oxygen species via double staining. Neurosci Lett. 2006; 399:206-209. [PubMed: 16530963]

34. Chen F, Sun X, De Keyzer F, Yu J, Peeters R, Coudyzer W, Vandecaveye V, Bosmans H, Van Hecke P, Landuyt W, Hermans R, Marchal G, Ni Y. Liver tumor model with implanted rhabdomyosarcoma in rats: MR imaging, microangiography, and histopathologic analysis. Radiology. 2006; 239:554-562. [PubMed: 16543589]

35. Wang HJ, Miranda Cona M, Chen F, Li JJ, Yu J, Feng YB, Peeters R, De Keyzer F, Marchal G, Ni Y. Comparison between nonspecific and necrosis-avid Gadolinium contrast agents in therapeutic necrosis of rodent tumors at 3.0T. Invest Radiol. 2011; 46:531-538. [PubMed: 21577133] 
36. Kerns, EH.; Di, L. Drug-like Properties: Concepts, Structure Design and Methods. Academic Press; Burlington, MA: 2008. Plasma Stability Methods; p. 329-347.

37. Ackermann L, Barfuesser S, Potukuchi HK. Copper-catalyzed N-arylation/hydroamin(d)ation domino synthesis of indoles and its application to the preparation of a Chek1/KDR kinase inhibitor pharmacophore. Adv Synth Catal. 2009; 351:1064-1072.

38. Bellina F, Cauteruccio S, Rossi R. Palladium- and copper-mediated direct C-2 arylation of azoles -including free $(\mathrm{NH})$-imidazole, -benzimidazole and -indole- under base-free and ligand-less conditions. Eur J Org Chem. 2006; 6:1379-1382.

39. Lipinska TM. Total synthesis of new indolo[2,3-a]quinolizine alkaloids sempervirine type, potential pharmaceuticals. Tetrahedron. 2006; 62:5736-5747.

40. Sakai H, Tsutsumi K, Morimoto T, Kakiuchi K. One-pot/four-step/palladium-catalyzed synthesis of indole derivatives: the combination of heterogeneous and homogeneous systems. Adv Synth Catal. 2008; 350:2498-2502.

41. Jin Z, Guo SX, Qiu LL, Wu GP, Fang JX. Well-defined NHC-Pd complex-mediated intermolecular direct annulations for synthesis of functionalized indoles ( $\mathrm{NHC}=\mathrm{N}$-hetero-cyclic carbene). Appl Organomet Chem. 2011; 25:502-507.

42. Nador F, Moglie Y, Vitale C, Yus M, Alonso F, Radivoy G. Reduction of polycyclic aromatic hydrocarbons promoted by cobalt or manganese nanoparticles. Tetrahedron. 2010; 66:4318-4325.

43. Klioze SS, Ehrgott FJ Jr, Glamkowski EJ. Synthesis of 7-substituted $1 H$-indolo[3, 2- $d][1$, 2]benzoxazepines. J Heterocycl Chem. 1984; 21:1257-1259.

44. Shen M, Leslie BE, Driver TG. Dirhodium(II)-catalyzed intramolecular C-H amination of aryl azides. Angew Chem, Int Ed. 2008; 47:5056-5059.

45. So CM, Lau CP, Kwong FY. Easily accessible and highly tunable indolyl phosphine ligands for Suzuki-Miyaura coupling of aryl chlorides. Org Lett. 2007; 9:2795-2798. [PubMed: 17602563]

46. Kraus GA, Guo H. One-pot synthesis of 2-substituted indoles from 2-aminobenzyl phosphonium salts. A formal total synthesis of arcyriacyanin A. Org Lett. 2008; 10:3061-3063. [PubMed: 18572918]

47. Kissman HM, Farnsworth DW, Witkop B. Fischer indole syntheses with polyphosphoric acid. J Am Chem Soc. 1952; 74:3948-3949.

48. De Cointet P, Pigerol C, Broll M, Eymard P, Werbenec JP. Synthesis and pharmacological properties of 3-acetoacetylindoles and their derivatives. Eur J Med Chem. 1976; 11:471-479.

49. Adkins H, Coonradt HL. Selective hydrogenation of derivatives of pyrrole, indole, carbazole and acridine. J Am Chem Soc. 1941; 63:1563-1570.

50. Slusasrchyk, WA.; Bolton, SA.; Herpin, T.; Bisacchi, GS.; Pi, Z.; Priestley, ES. Preparation of Oxazolylureidoanilines as Inhibitors of Serine Proteases Such as Factor VIIa. PCT. Int. Appl. WO 2004000788 A1. Dec 31. 2003

51. Kumar S, Gawandi VB, Capito N, Phillips RS. Substituent effects on the reaction of $\beta$ benzoylalanines with Pseudomonas fluorescens kynureninase. Biochemistry. 2010; 49:7913-7919. [PubMed: 20690660]

52. Lutz RE, Allison RK, Ashburn G, Bailey PS, Clark MT, Codington JF, Deinet AJ, Freek JA, Jordan RH, Leake NH, Martin TA, Nicodemus KC, Rowlett RJ Jr, Shearer NH Jr, Smith JD, Wilson JW III. Antimalarials. a-Phenyl- $\beta$-dialkylamino alcohols. J Org Chem. 1947; 12:617-703. [PubMed: 20264591]

53. McCoubrey A, Iyengar NK. $a$-Methylbenzylamines. II. 3-Cyclohexyloxy-4-hydroxy- $a$ methylbenzylamine, its desoxy derivative, and related ethers. J Chem Soc. 1951:3430-3433.

54. Imran M, Dudhe R, Sharma PK, Khan SA. Synthesis and anticonvulsant activity of 3-chloro-4substituted phenyl-1-[\{4-(1-naphthyl)-1,3-thiazol-2-yl \}amino]azetidin-2-ones. Int J Chem Sci. 2007; 5:189-200.

55. (a) Grammaticakis P. Remarks about the preparation and ultraviolet absorption of some $o-, m-$, and p-methylbenzoylarylamines. Compt Rend. 1962; 255:1456-1458.(b) Ma Y, Song C, Chai Q, Ma C, Andrus MB. Palladium-imidazolium N-heterocyclic carbene-catalyzed carbonylative amidation with boronic acids, aryl diazonium ions, and ammonia. Synthesis. 2003; 18:2886-2889.

56. Bachmann WE, Barton MX. The relative proportions of stereoisomeric oximes formed in the oximation of unsymmetrical ketones. J Org Chem. 1938; 3:300-311. 
57. Crowther AF, Mann FG, Purdie D. Mechanism of indole formation from phenacylarylamines. I. J Chem Soc. 1943:58-68.

58. Harej M, Dolenc D. Autoxidation of hydrazones. Some new insights. J Org Chem. 2007; 72:7214 7221. [PubMed: 17696476]

59. Hamel E. Evaluation of antimitotic agents by quantitative comparisons of their effects on the polymerization of purified tubulin. Cell Biochem Biophys. 2003; 38:1-21. [PubMed: 12663938]

60. Verdier-Pinard P, Lai JY, Yoo HD, Yu J, Marquez B, Nagle DG, Nambu M, White JD, Falck JR, Gerwick WH, Day BW, Hamel E. Structure-activity analysis of the interaction of curacin A, the potent colchicine site antimitotic agent, with tubulin and effects of analogs on the growth of MCF-7 breast cancer cells. Mol Pharmacol. 1998; 35:62-76. [PubMed: 9443933]

61. Ruan S, Okcu MF, Pong RC, Andreeff M, Levin V, Hsieh JT, Zhang W. Attenuation of WAF1/ Cip1 expression by an antisense adenovirus expression vector sensitizes glioblastoma cells to apoptosis induced by chemotherapeutic agents 1,3-bis(2-chloroethyl)-1-nitro-sourea and cisplatin. Clin Cancer Res. 1999; 5:197-202. [PubMed: 9918219]

62. Abramoff MD, Magelhaes PJ, Ram SJ. Image processing with image. Biophotonics Int. 2004; 11:36-42.

63. Barzegar A, Moosavi-Movahedi AA. Intracellular ROS protection efficiency and free radicalscavenging activity of curcumin. PLoS One. 2011; 6:e26012. [PubMed: 22016801] 

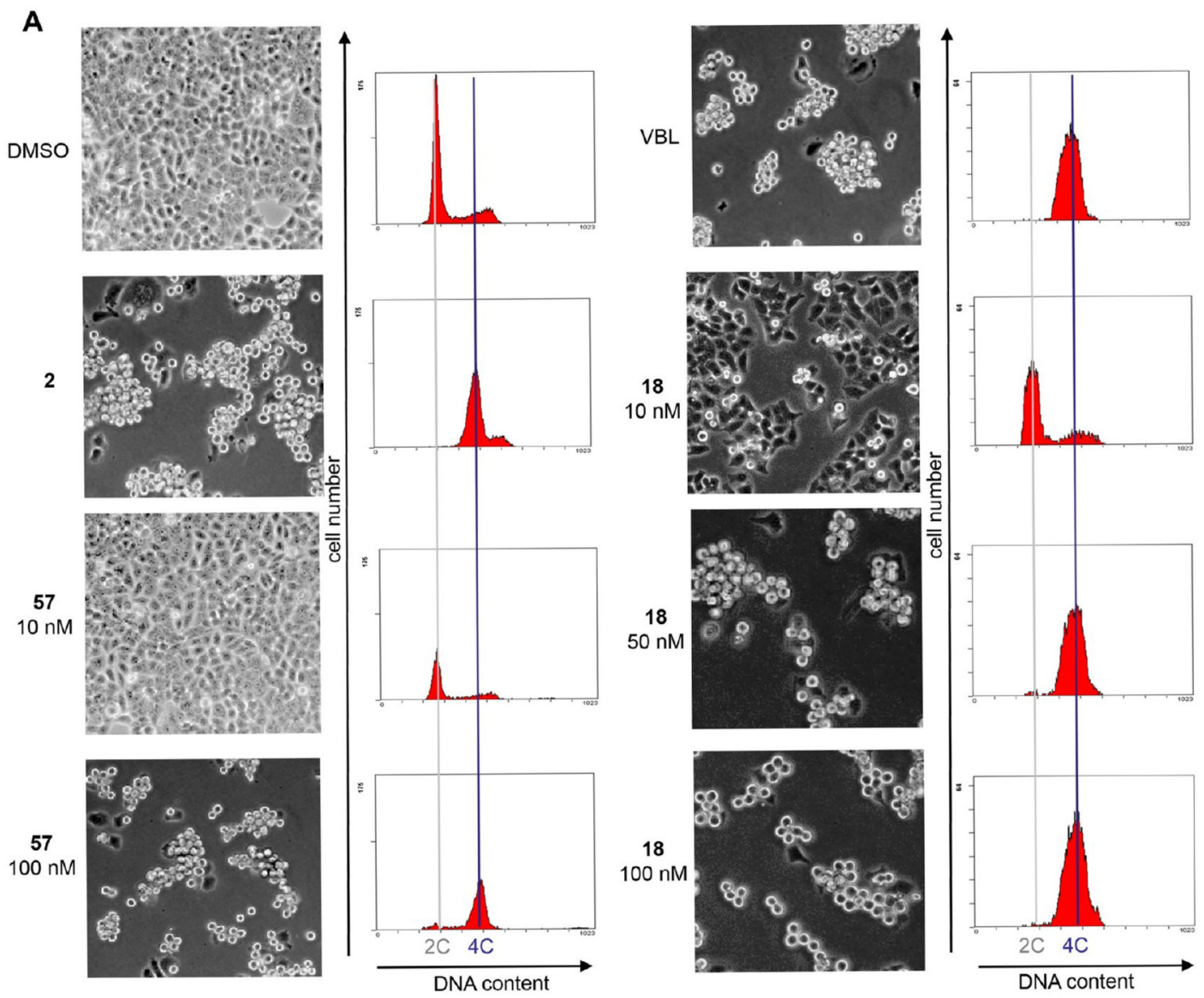

B

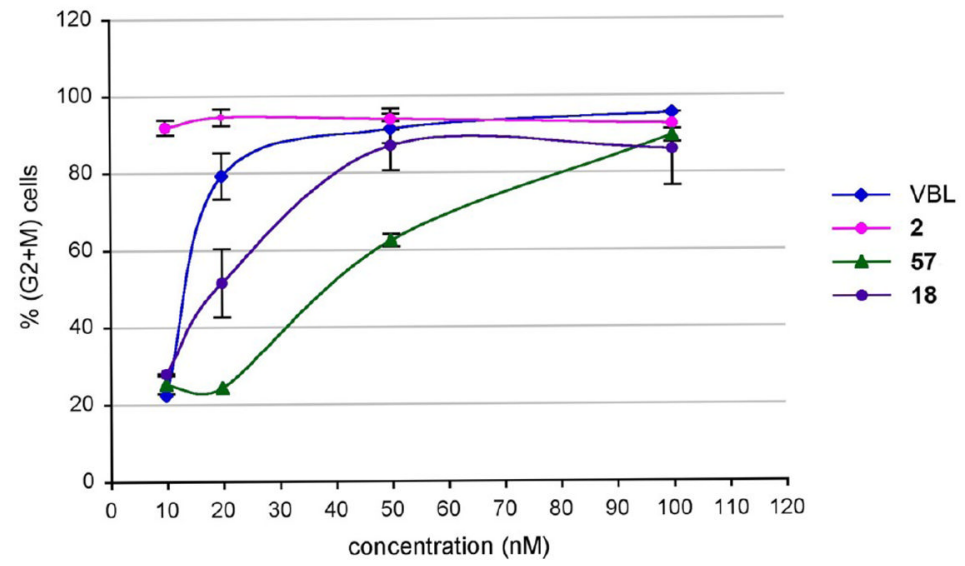

Figure 1.

(A) Analysis of HeLa cell cultures treated with $\mathbf{1 8}$ or $\mathbf{5 7}$ for $24 \mathrm{~h}$ by wide-field microscopy (left panels) and flow cytometry (right panels). The left panels in each group show representative fields from unfixed cultures (10× objective): cells arrested in mitosis detach from the culture dish surface, leaving ample empty spaces, and are recognizable from their rounded-up refractive morphology. Cycling cultures are adherent with only rare rounded mitotic cells. Flow cytometry profiles of the cultures are shown on the right. The histrograms represent the ditribution of cells according to their DNA content calculated from the emitted PI fluorescence. Fluorescence intensity values are plotted on the $x$ axis and cell numbers are plotted on the $y$ axis. The $2 \mathrm{C}$ peak (in gray) identifies G1 cells. The $4 \mathrm{C}$ peak (in 
blue) identifies G2/M cells. Cells with intermediate DNA content are in S phase. (B) Mean frequency and SD of the frequency of cells with a $4 \mathrm{C}$ content $(\mathrm{G} 2+\mathrm{M})$ under the indicated conditions. From three to five assays were carried out for every treatment, and data from 20 000 cells per assay were acquired. 
A

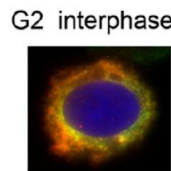

DAPI $\alpha$-tubulin

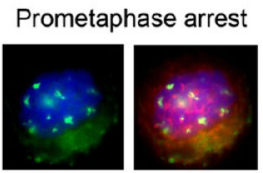

DAPI $\alpha$-tubulin merge w cyclin B1
Multinucleated cell

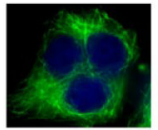

DAPI $\alpha$-tubulin

B

18

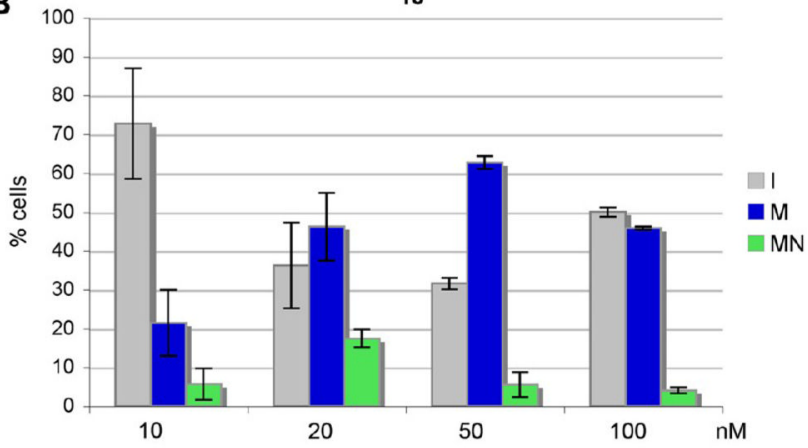

57

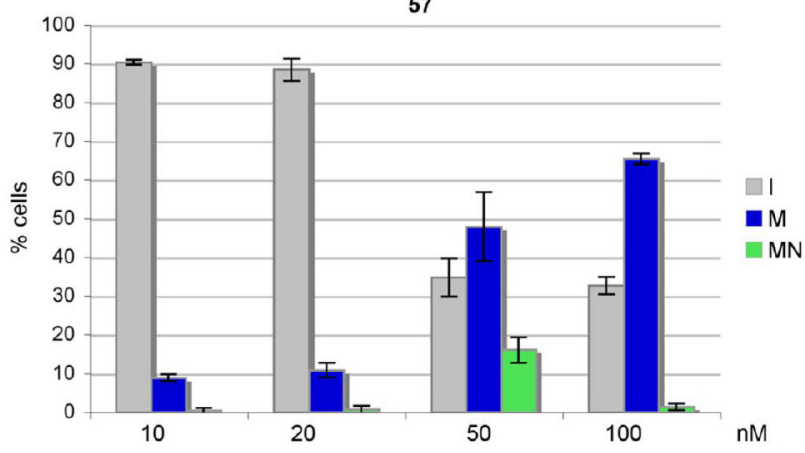

Figure 2.

(A) Examples of immunofluorescenctly stained cells. In all panels, the DNA is blue (stained with DAPI), $\boldsymbol{a}$-tubulin is green, and cyclin B1 is red, as indicated. Left: A G2 interphase cell identified by cyclin B1 expression. Middle: A cell arrested in prometaphase with depolymerized microtubules (shown with and without merging with cyclin B1 staining). Right: A multinucleated cell derived from mitotic slippage. (B) Distribution of cells in doseresponse experiments with $\mathbf{1 8}$ and $\mathbf{5 7}$. The histograms represent the mean frequency, and bars represent the SD of interphases (I), mitoses (M), and multinculeated cells (MN) from three independent experiments. 

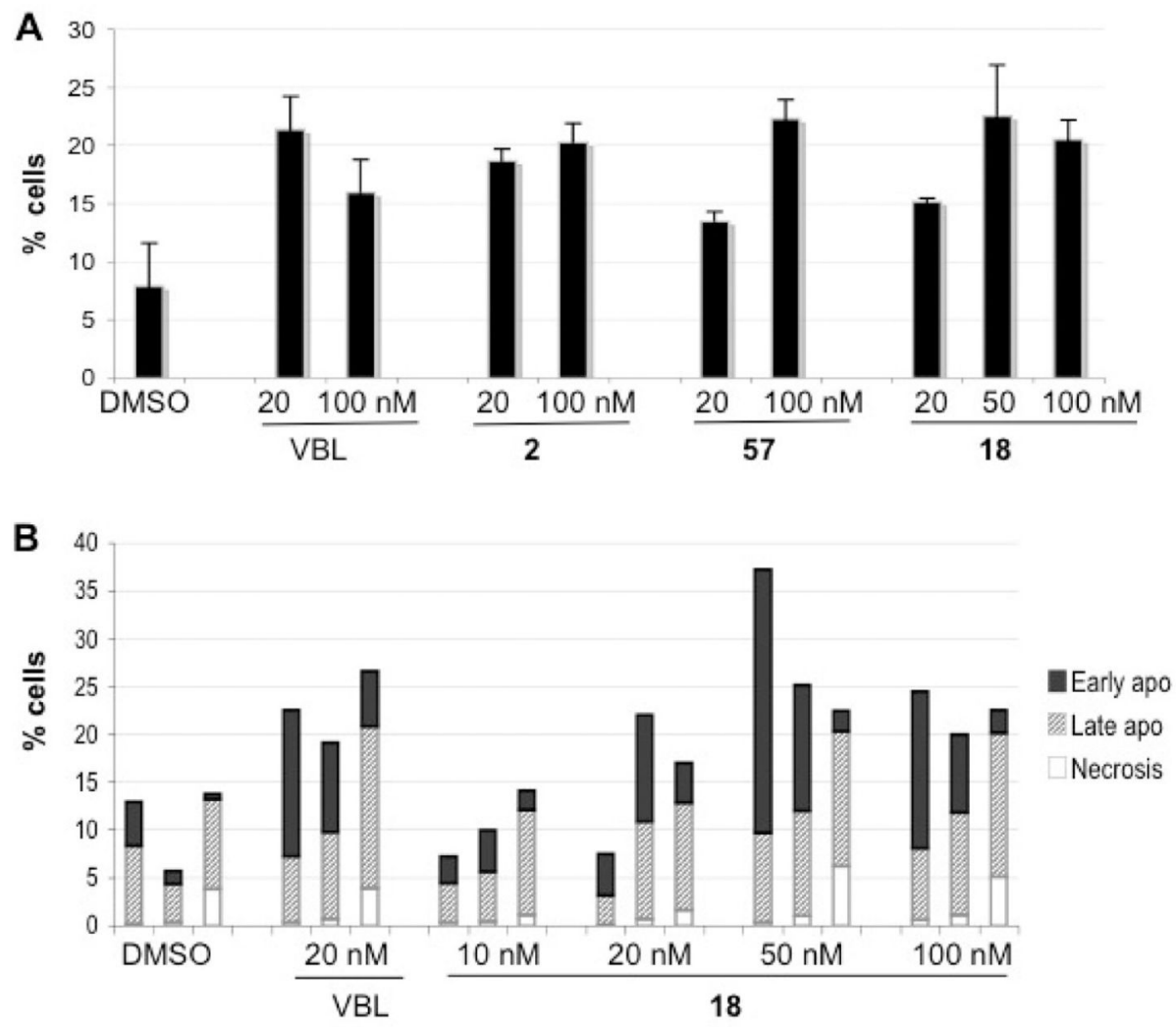

Figure 3.

(A) Frequency of apoptotic cells in cultures treated with the indicated concentrations of $\mathbf{1 8}$ or 57. The histograms represent mean frequencies, and bars represent the SDs of annexin V reactive cells from three independent experiments. (B) Biparametric analysis of cell death in dose-response experiments with 18. Early apoptotic, late apotptotic, and necrotic cells are distinguished by their differential reactivity to annexin V but not PI, to both annexin V and PI, and to PI but not annexin V, respectively. Results from three independent experiments are shown for each concentration. No condition induced significant necrosis compared with the baseline level seen in DMSO-treated controls (data from 20000 cells acquired per sample). 


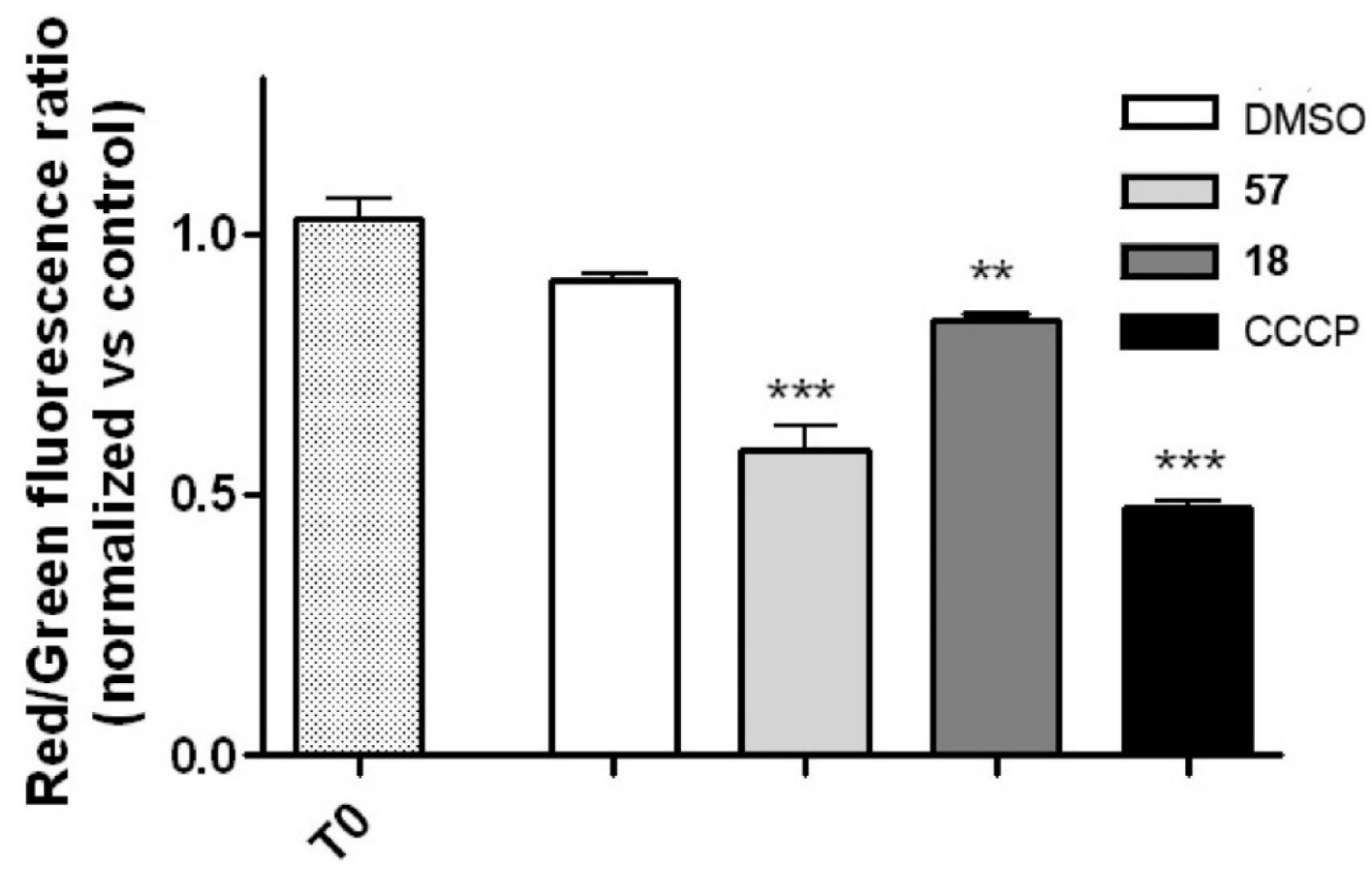

Figure 4.

Effects of a $24 \mathrm{~h}$ treatment with $100 \mathrm{nM} 57$ or 18 or $30 \mu \mathrm{M}$ CCCP on mitochondrial transmembrane potential as assessed by JC-1 staining. Graphs indicate the $\Delta \Psi$ dissipation expressed as red/green $(\mathrm{R} / \mathrm{G})$ fluorescence ratio. Each value has been normalized versus the $\mathrm{R} / \mathrm{G}$ ratio of the vehicle treated control to which an arbitrary value of $100 \%$ has been assigned. Data were the mean of two independent experiments performed in triplicate $\left(\left(^{* *}\right)\right.$ $p<0.01$ and $(* * *) p<0.001$, one-way ANOVA, Bonferroni's corrected $t$ test for post hoc pairwise comparisons). 

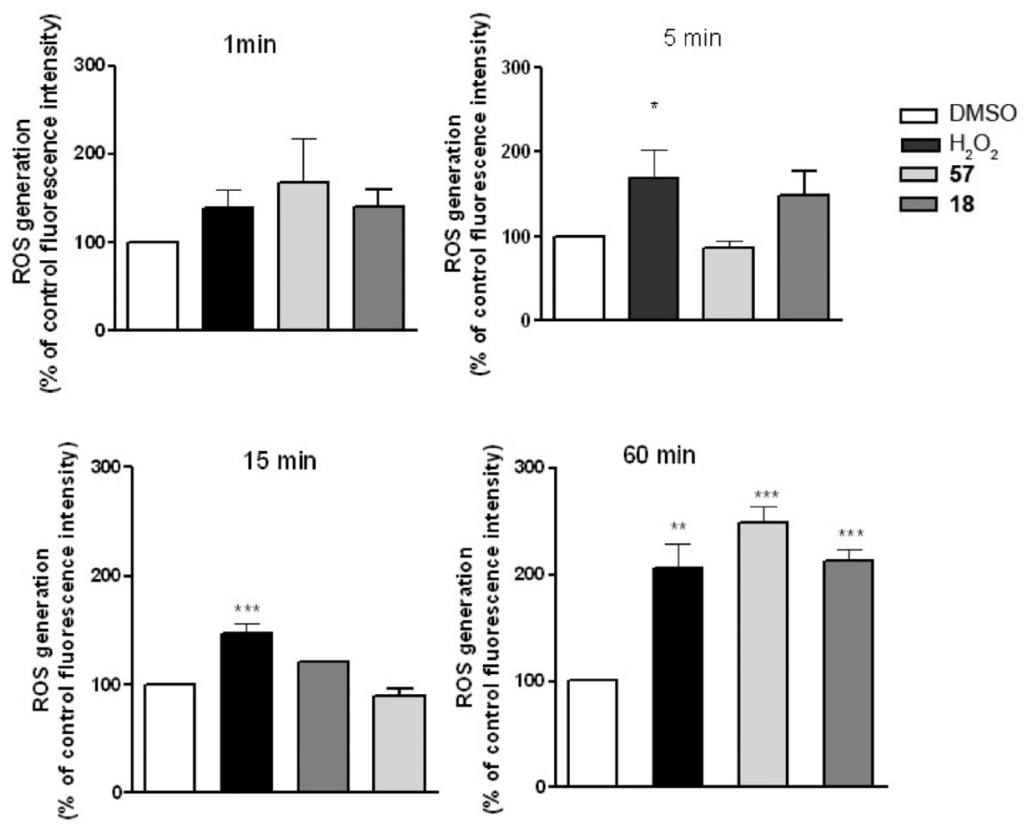

Figure 5.

Effects of treatment with $100 \mathrm{nM} 57$ or 18 and $400 \mathrm{mM} \mathrm{H}_{2} \mathrm{O}_{2}$ on ROS formation in U87MG cells after exposures of 1, 5, 15, and $60 \mathrm{~min}$. The results are expressed as \% of mean fluorescence intensity relative to the control and normalized for cell number. Data were the mean of two independent experiments performed in triplicate $\left(\left(^{*}\right) p<0.05,(* *) p<0.01\right.$, and $(* * *) p<0.001$, one-way ANOVA, Bonferroni's corrected $t$ test for post hoc pairwise comparisons). 

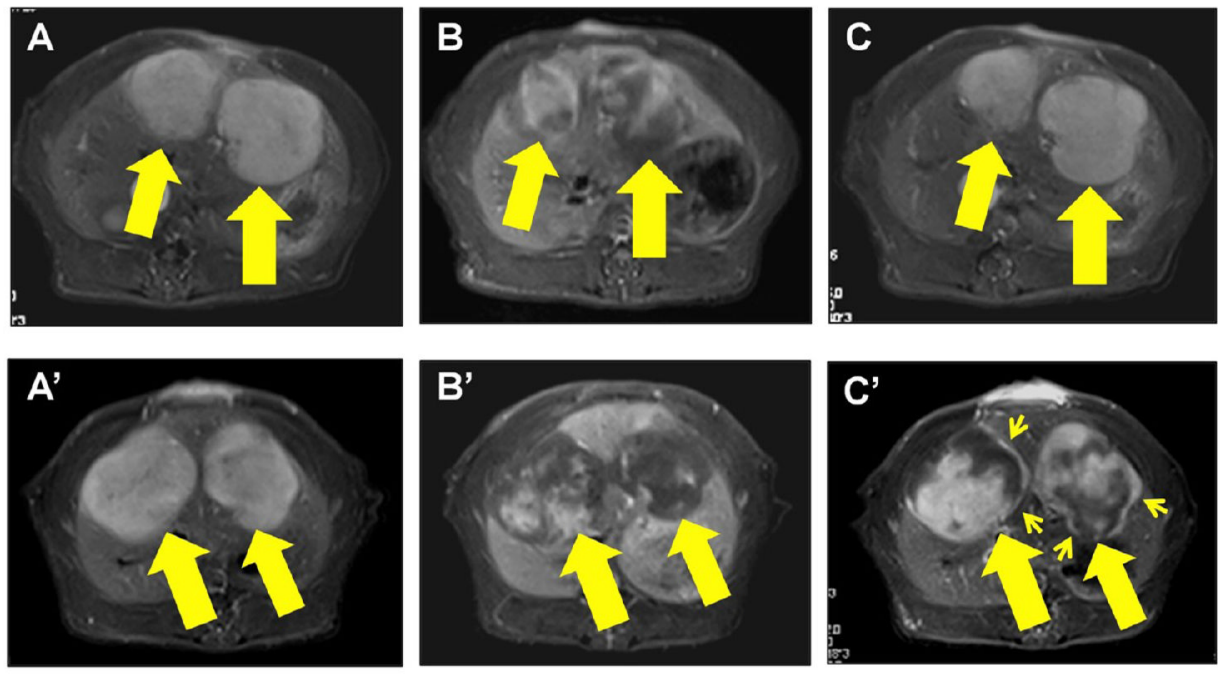

Figure 6.

Contrast enhanced MRI was used in rats with bifocal liver growths of rhabdomyosarcoma (smaller and larger arrows denote smaller and larger lesions, respectively). At baseline prior to treatment, the liver tumors appear as homogeneous hyperintense spheroidal nodules, suggesting their hypervascularity (A, $\left.A^{\prime}\right)$. At $1 \mathrm{~h}$ after intravenous injection of either 57 (B) or $18\left(\mathrm{~B}^{\prime}\right)$, the tumors were partially enhanced because of their early vascular disrupting effects, which appeared stronger with 18 (B') than $\mathbf{5 7}(\mathrm{B})$. At $24 \mathrm{~h}$ after treatment, the tumors treated with $\mathbf{5 7}$ were enhanced completely $(\mathrm{C})$, suggesting only transient early vascular disruption effect and the absence of any consequent tumor necrosis. However, tumors treated with $\mathbf{1 8}$ showed rim (arrowheads) and partial central enhancement $\left(\mathrm{C}^{\prime}\right)$, suggesting the presence of both tumor necrosis and viable residues. 


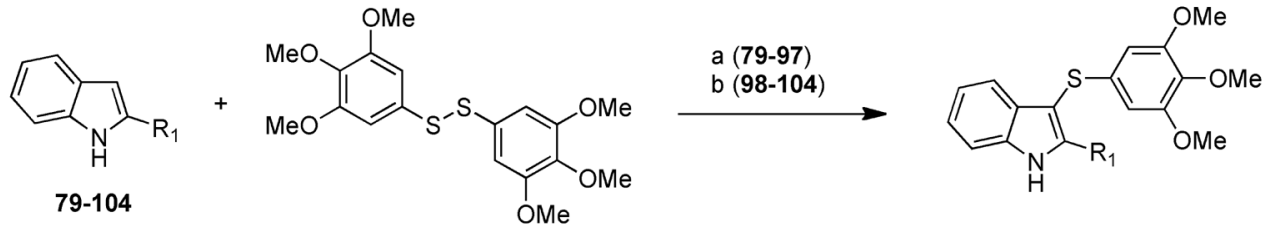

a: $17,18,20,27,33,34,38,39,41,43,45-47,53,55,57,73,75,77 ;$ b: $14,16,37,48,59,62,72$<smiles>COc1cc(SSc2cc(OC)c(OCC(=O)O)c(OC)c2)cc(OC)c1OC</smiles>
$106(\mathrm{R}=\mathrm{l})$<smiles>[R2]c1[nH]c2ccccc2c1Sc1cc(OC)c(OC)c(OC)c1</smiles><smiles>[R]C#[R]C</smiles><smiles>COc1cc(Sc2c(I)[nH]c3ccccc23)cc(OC)c1OC</smiles>

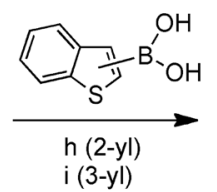<smiles></smiles>
67: $R_{2}=$ indol-5-yl<smiles>COc1cc(Sc2[nH]c3ccccc3c2-c2cc3ccccc3[nH]2)cc(OC)c1OC</smiles>

68
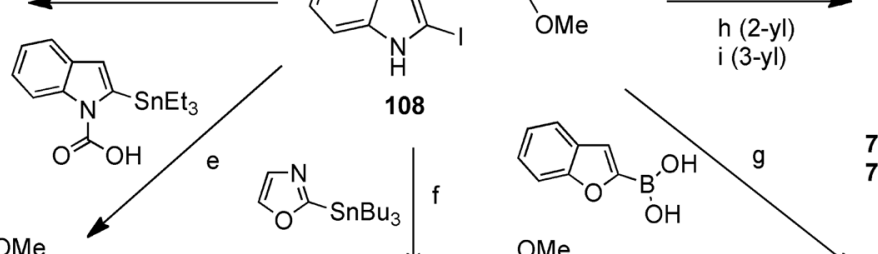

71 (thiophen-3-yl)

Scheme $1^{\text {a }}$

${ }^{a}$ Reagents and reaction conditions: (a) (i) indoles 79-97 $\left(\mathrm{R}_{1}=\right.$ imidazol-2-yl (79), imidazol-1-yl (80), ${ }^{21}$ 1,2,4-triazol-1-yl (81), thiazol-2-yl (82), 4,5-dihydro-1 $H$-imidazol-2-yl (83), phenyl (84), 4-chlorophenyl (85), 2-fluorophenyl (86), 3-fluorophenyl (87), 4fluorophenyl (88), 2-methoxyphenyl (89), 3-methoxyphenyl (90), 4-methoxyphenyl (91), pyridin-2-yl (92), pyridin-3-yl (93), pyridin-4-yl (94), cyclobutyl (95), cyclopentyl (96), cyclohexyl (97)), $\mathrm{NaH}$, anhydrous DMF, $25^{\circ} \mathrm{C}, 10 \mathrm{~min}$; (ii) closed vessel, $110^{\circ} \mathrm{C}, 150 \mathrm{~W}, 2$ min, yield 3-77\%; (b) (i) indoles 98-104 $\left(\mathrm{R}_{1}=\right.$ pyrazol-4-yl (98), pyrazol-3-yl (99), ${ }^{22} 4$ tolyl (100), 3-isopropoxy-4-methoxyphenyl (101), naphth-1-yl (102), naphth-2-yl (103), 1,1' $1^{\prime}$-biphenyl-4-yl (104), $\left.\mathrm{COOH}(\mathbf{1 0 5}), \mathrm{I}(\mathbf{1 0 6})^{23}\right)$, NaH, anhydrous DMF, $0{ }^{\circ} \mathrm{C}, 15 \mathrm{~min}, \mathrm{Ar}$ stream; (ii) $60^{\circ} \mathrm{C}, 12 \mathrm{~h}$, Ar stream, 35-72\%; (c) $\mathrm{Et}_{3} \mathrm{~N}$, BOP reagent, anhydrous DMF, 25 ${ }^{\circ} \mathrm{C}, 2 \mathrm{~h}, 30 \%$; (d) 6-quinolineboronic acid pinacol ester (for 66) or 1-Boc-indole-5-boronic acid pinacol ester (for 67), $\mathrm{Pd}(\mathrm{dppf}) \mathrm{Cl}_{2} \cdot \mathrm{CH}_{2} \mathrm{Cl}_{2}, \mathrm{~K}_{3} \mathrm{PO}_{4}$, anhydrous DMF, Ar stream, 110 ${ }^{\circ} \mathrm{C}, 12 \mathrm{~h}$, yield 26-70\%; (e) 2-(triethylstannyl)-1 $H$-indole-1-carboxylic acid ${ }^{24} \mathrm{PdCl}_{2}\left(\mathrm{PPh}_{3}\right)_{2}$, ethanol, reflux, $78{ }^{\circ} \mathrm{C}, 48 \mathrm{~h}$, Ar stream, yield 20\%; (f) 2-(tributylstannyl)oxazole, $\mathrm{Pd}_{2}\left(\mathrm{dba}_{3}\right.$, $\mathrm{P}(\mathrm{Cy})_{3}$, anhydrous DMF, closed vessel, $150{ }^{\circ} \mathrm{C}, 150 \mathrm{~W}, 10 \mathrm{~min}$, yield $35 \%$; (g) 2benzofuranylboronic acid, $\mathrm{Pd}(\mathrm{OCOMe})_{2}, \mathrm{~K}_{3} \mathrm{PO}_{4}, 1,4$-dioxane $/ \mathrm{H}_{2} \mathrm{O}, 100{ }^{\circ} \mathrm{C}, 24 \mathrm{~h}, \mathrm{Ar}$ stream, yield 88\%; (h) benzo[b]thien-2-ylboronic acid, $\mathrm{Pd}\left(\mathrm{PPh}_{3}\right)_{4}, \mathrm{Na}_{2} \mathrm{CO}_{3}$, THF, $60{ }^{\circ} \mathrm{C}, 24$ h, Ar stream, yield 10\%; (i) benzo[b]thien-3-ylboronic acid, $\mathrm{Pd}_{2}(\mathrm{dba})_{3}, \mathrm{SPhos}, \mathrm{K}_{3} \mathrm{PO}_{4}, n-$ $\mathrm{BuOH}, \mathrm{Ar}$ stream, $100^{\circ} \mathrm{C}, 12 \mathrm{~h}$, yield $36 \%$. 


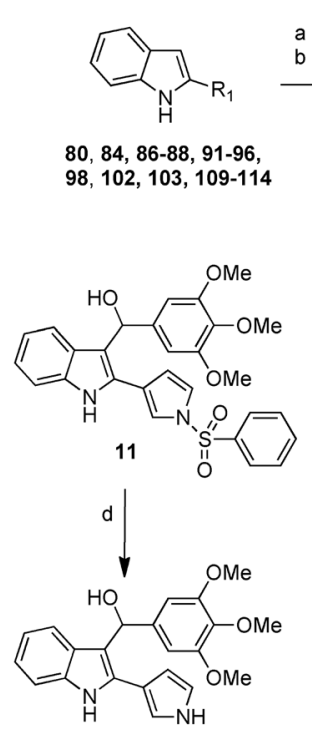

10 a $(80,92-94,98,102,109)$ b $(84,86-88,91,94-96,103,110-114)$

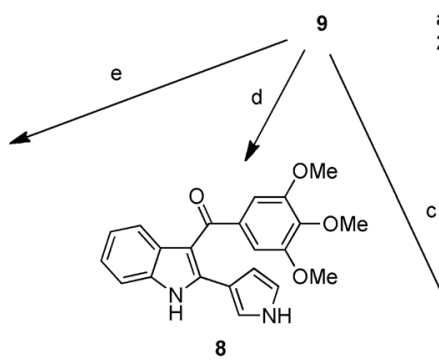

8

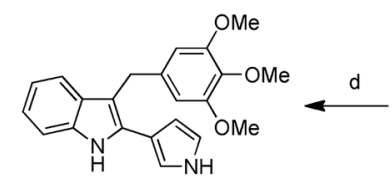

13

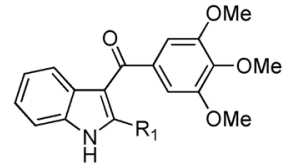

$7,15,19,54,56,58,60 ;$ b: $9,21,23$ $25,32,35,40,42,44,51,63,74,76,78$

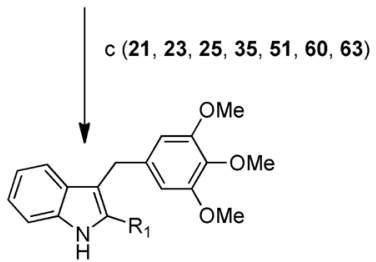

$22,24,26,36,52,61,65$

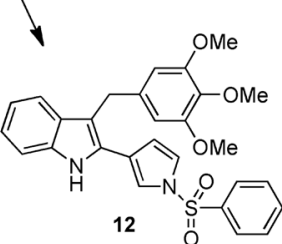

Scheme $2^{\mathbf{a}}$

${ }^{a}$ Reagents and reaction conditions: (a) (i) indoles 80, 92-94, 98, 102, $109\left(\mathrm{R}_{1}=\right.$ imidazol-1yl (80), ${ }^{21}$ pyridin-2-yl (92), pyridin-3-yl (93), pyridin-4-yl (94), pyrazol-4-yl (98), naphth-1yl (102), pyrrol-2-yl (109), ${ }^{25} \mathrm{MeMgBr}$, anhydrous $\mathrm{ZnCl}_{2}$, anhydrous $\mathrm{CH}_{2} \mathrm{Cl}_{2}, 25^{\circ} \mathrm{C}, 1 \mathrm{~h}, \mathrm{Ar}$ stream; (ii) 3,4,-5-trimethoxybenzoyl chloride, $25{ }^{\circ} \mathrm{C}, 1 \mathrm{~h}$, Ar stream; (iii) $\mathrm{SnCl}_{4}, 25^{\circ} \mathrm{C}, 12$ h, Ar stream, yield 3-67\%; (b) indoles 86-88, 91, 94-96, 103, 110-114 $\left(\mathrm{R}_{1}=\right.$ phenyl $(\mathbf{8 4})$, 2-fluorophenyl (86), 3-fluorophenyl (87), 4-fluorophenyl (88), 4-methoxyphenyl (91), cyclobutyl (94), cyclopentyl (95), cyclohexyl (96), naphth-2-yl (103), 1(phenylsulfonyl)-1 $H$-pyrrol-3-yl (110), ${ }^{19}$ furan-2-yl (111), ${ }^{19}$ furan-3-yl (112),${ }^{19}$ thiophen-3yl (113), ${ }^{19}$ oxazol-2-yl (114)), 3,4,5-trimethoxybenzoyl chloride, anhydrous $\mathrm{AlCl}_{3}, 1,2$ dichloroethane, closed vessel, $110^{\circ} \mathrm{C}, 150 \mathrm{~W}, 2$ min, yield 5-95\%; (c): $\mathrm{BH}_{3} / \mathrm{THF}, \mathrm{MeCN} /$ $\mathrm{MeOH}, 50{ }^{\circ} \mathrm{C}, 1 \mathrm{~h}$, Ar stream, yield 4-99\%; (d) $2 \mathrm{M} \mathrm{NaOH}, \mathrm{MeOH}$, reflux, $3 \mathrm{~h}$, yield 20$98 \%$; (e) $\mathrm{NaBH}_{4}, \mathrm{THF} / \mathrm{H}_{2} \mathrm{O}, 80{ }^{\circ} \mathrm{C}, 2 \mathrm{~h}$, yield $44-97 \%$. 

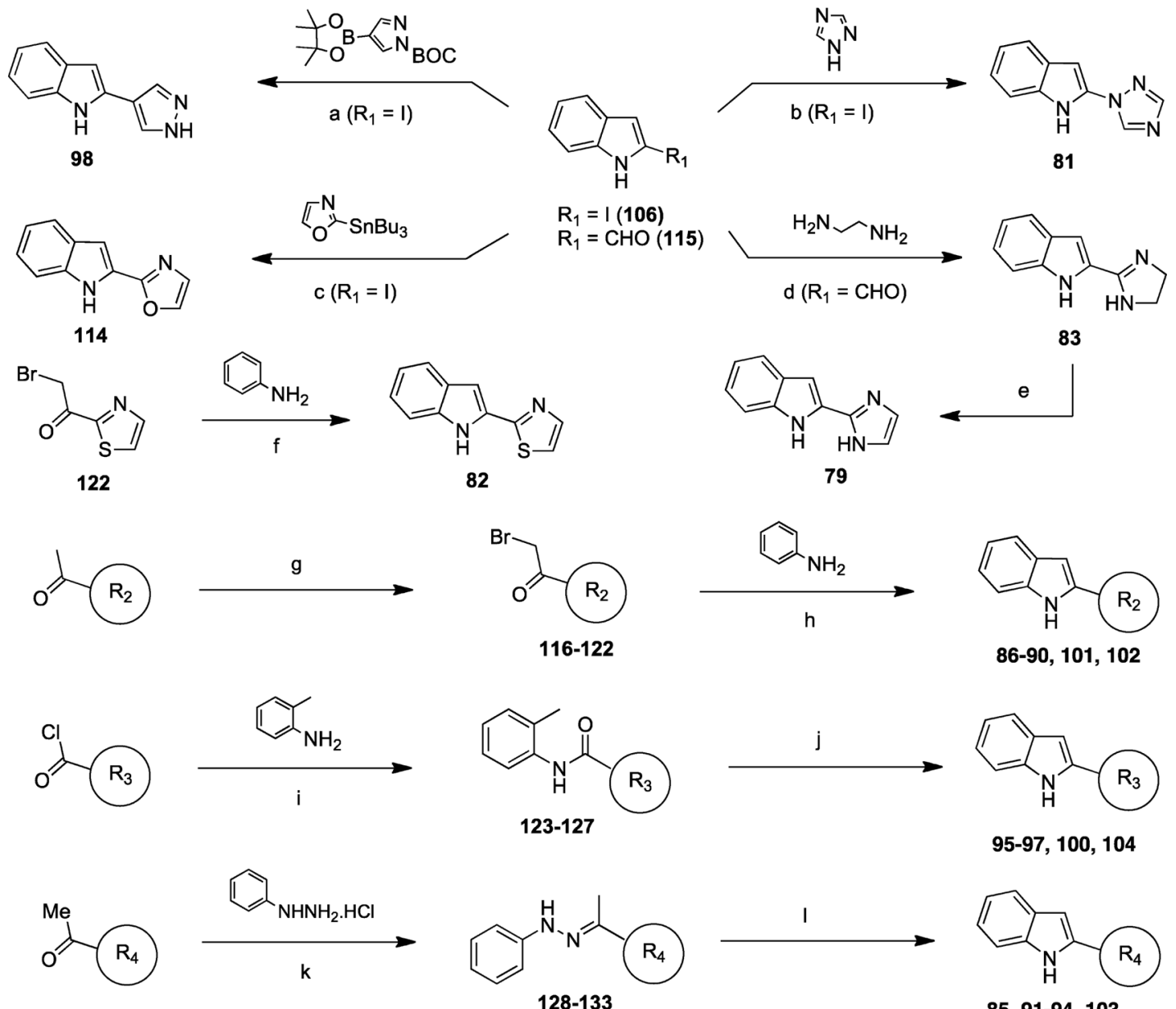

95-97, 100, 104

Scheme $3^{\mathrm{a}}$

${ }^{a} \mathrm{R}_{2}=2-\mathrm{F}-\mathrm{Ph}$ (86), 3-F-Ph (87), 4-F-Ph (88), 2-MeO-Ph (89), 3-MeO-Ph (90), 3-i-PrO-4$\mathrm{MeO}-\mathrm{Ph}$ (101), 1-naphthyl (102); $\mathrm{R}_{3}=$ cyclobutyl (95), cyclopentyl (96), cyclohexyl (97), 4Me-Ph (100), 1,1' -biphenyl-4-yl (104); $\mathrm{R}_{4}=4-\mathrm{Cl}-\mathrm{Ph}(\mathbf{8 5}), 4-\mathrm{MeO}-\mathrm{Ph}(\mathbf{9 1})$, pyridin-2-yl (92), pyridin-3-yl (93) pyridin-4-yl (94), 2-naphthyl (103). Reagents and reaction conditions: (a) $\mathrm{PdCl}_{2}\left(\mathrm{PPh}_{3}\right)_{2}, 1 \mathrm{M} \mathrm{Na}_{2} \mathrm{CO}_{3}, \mathrm{DMF}$, closed vessel, $160{ }^{\circ} \mathrm{C}, 250 \mathrm{~W}, 10 \mathrm{~min}$, yield 26\%; (b) $150{ }^{\circ} \mathrm{C}, 2 \mathrm{~h}$, yield $42 \%$; (c) $\mathrm{Pd}_{2}(\mathrm{dba})_{3}, \mathrm{P}(\mathrm{Cy})_{3}$, anhydrous DMF, closed vessel, $150{ }^{\circ} \mathrm{C}, 150$ $\mathrm{W}, 10 \mathrm{~min}$, yield $26 \%$; (d) (i) $t$ - $\mathrm{BuOH}$, Ar stream, $30 \mathrm{~min}$; (ii) $\mathrm{I}_{2}, \mathrm{~K}_{2} \mathrm{CO}_{3}, 70{ }^{\circ} \mathrm{C}, 3 \mathrm{~h}$, yield $43 \%$; (e) (diacetoxyiodo)benzene, $\mathrm{K}_{2} \mathrm{CO}_{3}$, DMSO, $25^{\circ} \mathrm{C}, 12 \mathrm{~h}$; (f) anhydrous DMF, closed vessel, $150{ }^{\circ} \mathrm{C}, 100 \mathrm{~W}, 1 \mathrm{~min}$, PowerMAX, yield $52 \%$; $(\mathrm{g}) \mathrm{Br}_{2}$, anhydrous $\mathrm{CH}_{2} \mathrm{Cl}_{2}, 25^{\circ} \mathrm{C}, 2$ h, yield 21-90\%; (h) $\mathrm{R}_{2}=2-\mathrm{F}$ (116), 3-F (117), 4-F (118), 2-MeO (119), ${ }^{28} 3-\mathrm{MeO}(\mathbf{1 2 0}),{ }^{28}$ 3-i-PrO-4-MeO (121), 1-naphthyl (122), N, $N$-dimethylaniline, $170{ }^{\circ} \mathrm{C}, 15 \mathrm{~min}$, yield $12-$ 42\%; (i) $\mathrm{R}_{3}=4$-tolyl (123), 1,1' -biphenyl-4-yl (124), cyclobutyl (125), cyclopentyl (126), cyclohexyl (127), anhydrous THF, $25^{\circ} \mathrm{C}, 12 \mathrm{~h}$, yield 40-90\%; (j) (i) $n$-BuLi, anhydrous $\mathrm{THF},-40{ }^{\circ} \mathrm{C}, 1 \mathrm{~h}$, Ar stream; (ii) $0{ }^{\circ} \mathrm{C}, 1 \mathrm{~h}$; (iii) $25^{\circ} \mathrm{C}, 12 \mathrm{~h}$, yield $22-59 \%$; (k) $\mathrm{R}_{4}=4-\mathrm{Cl}-\mathrm{Ph}$ (128), 4-MeO-Ph (129), pyridin-2-yl (130), ${ }^{29}$ pyridin-3-yl (131), ${ }^{29}$ pyridin-4-yl (132), ${ }^{29} 2$ naphthyl (133), ${ }^{29} \mathrm{CH}_{3} \mathrm{COONa}$, EtOH, open vessel, $250 \mathrm{~W}$, cooling-while-heating, $80{ }^{\circ} \mathrm{C}, 5$ min, yield 92-95\%; (1) polyphosphoric acid, $120{ }^{\circ} \mathrm{C}, 1 \mathrm{~h}$, yield $37-80 \%$. 

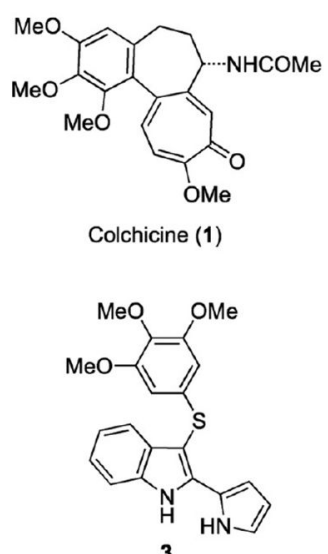
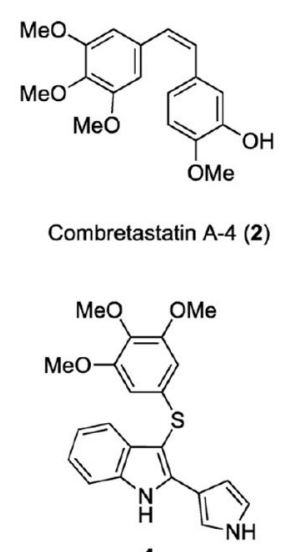
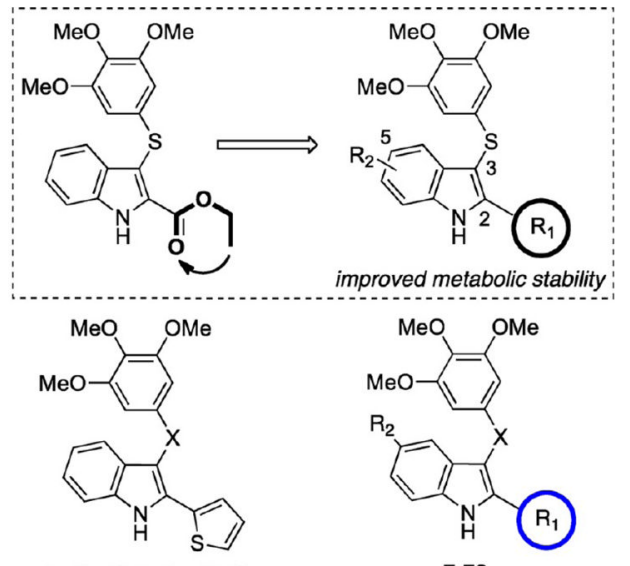

5: $X=S, 6: X=C=0$ pabolic stability

Chart 1. General Structure of ATI Derivatives 7-78 and Reference Compounds 1-6 ${ }^{\mathbf{a}}$ ${ }^{a} \mathrm{R}_{1}=$ azolyl, azinyl, phenyl, or substituted phenyl, naphthyl, biphenyl, benzofused heterocyclyl, alkyl, cycloalkyl; $\mathrm{R}_{2}=\mathrm{H} ; \mathrm{X}=\mathrm{S}, \mathrm{C}==\mathrm{O}, \mathrm{CHOH}$, or $\mathrm{CH}_{2}$ (Tables 1 and 2). 

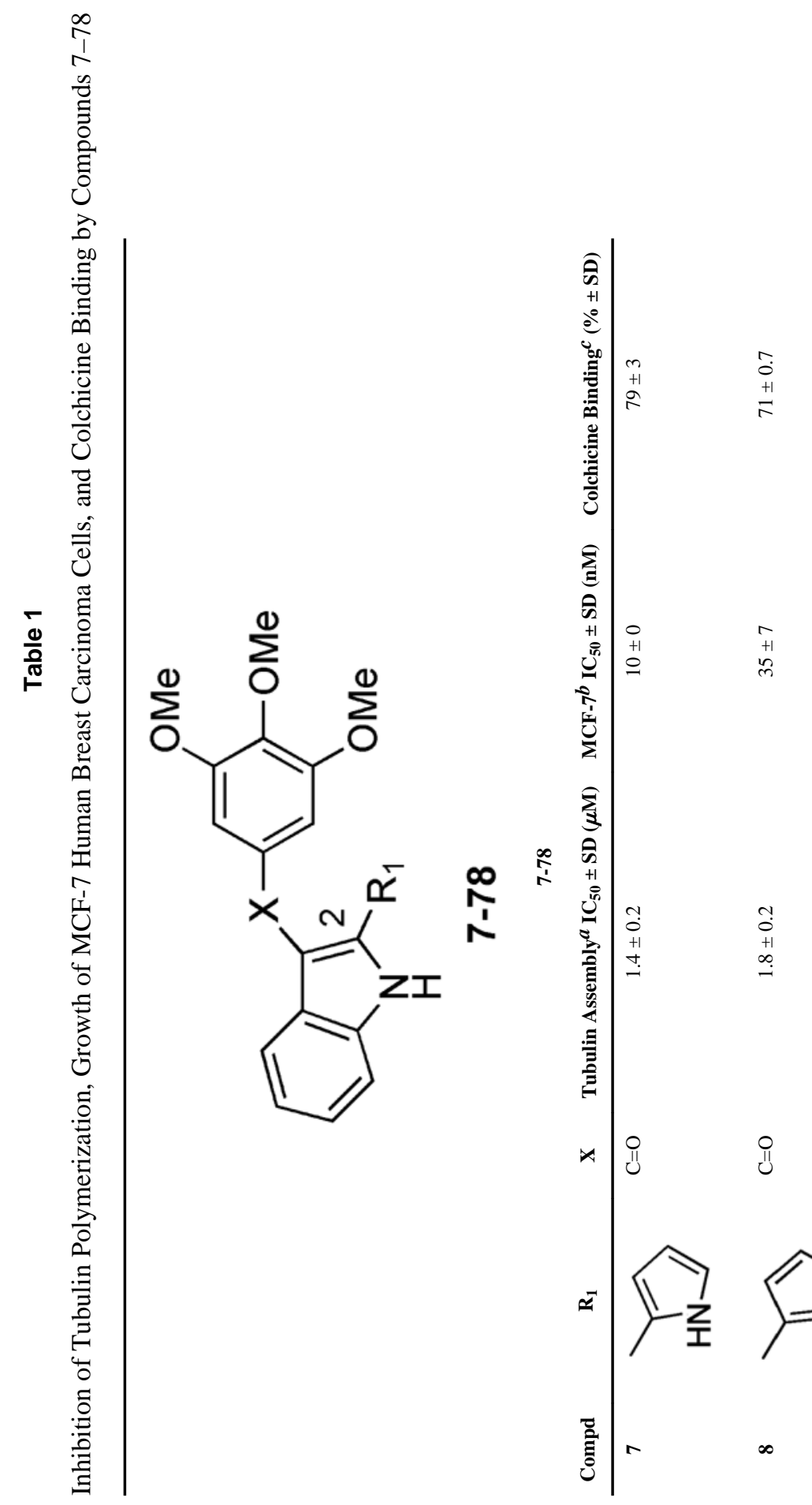

0
+1
en
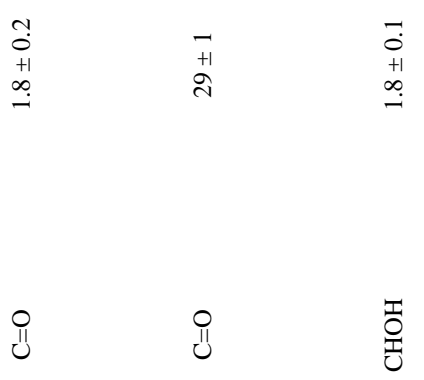

$\simeq$

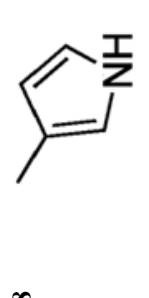<smiles></smiles><smiles>Cc1ccc(C)cc1</smiles>

JMed Chem. Author manuscript; available in PMC 2014 January 10. 


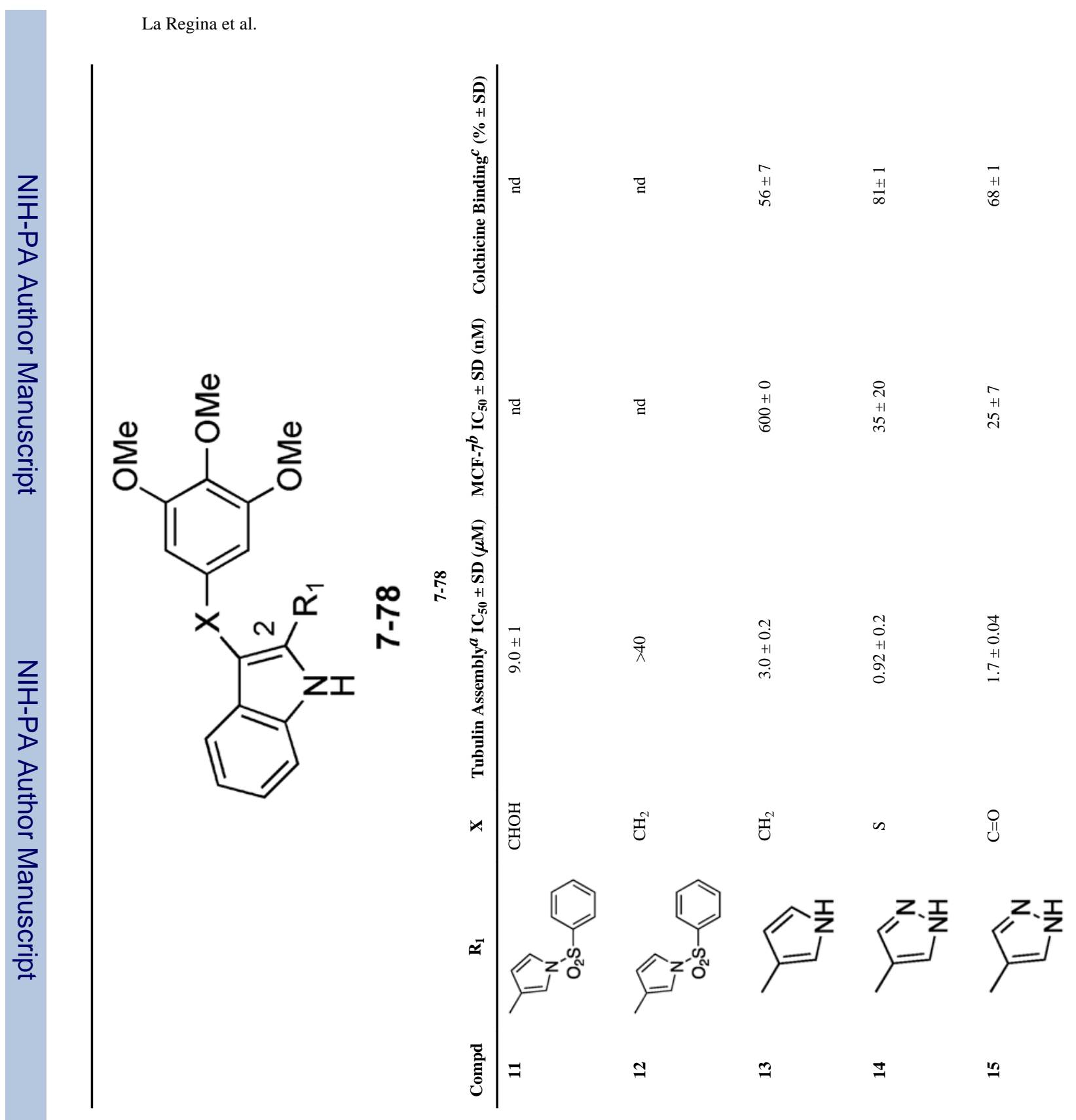




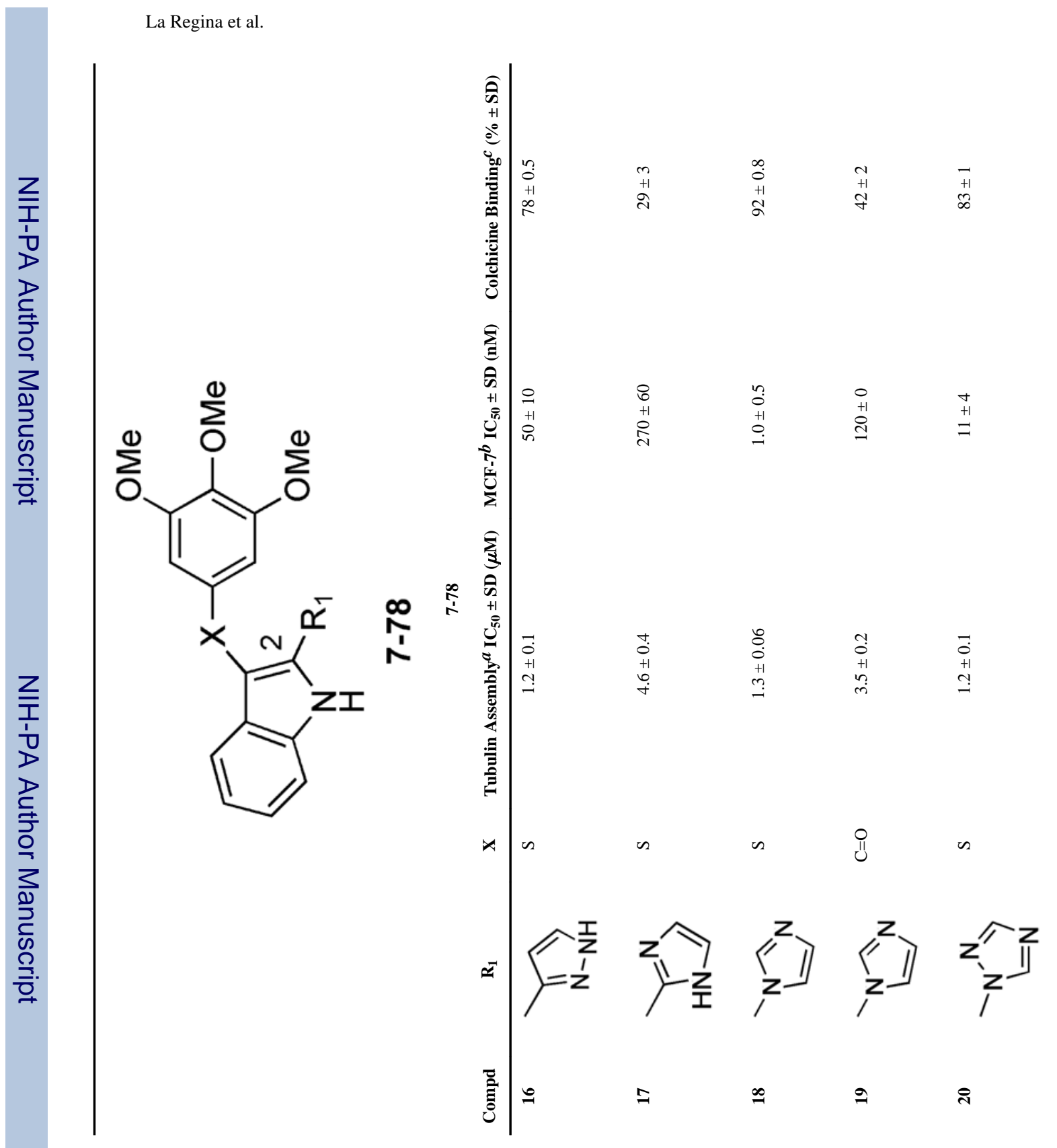

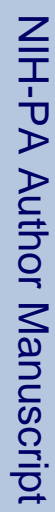

JMed Chem. Author manuscript; available in PMC 2014 January 10. 


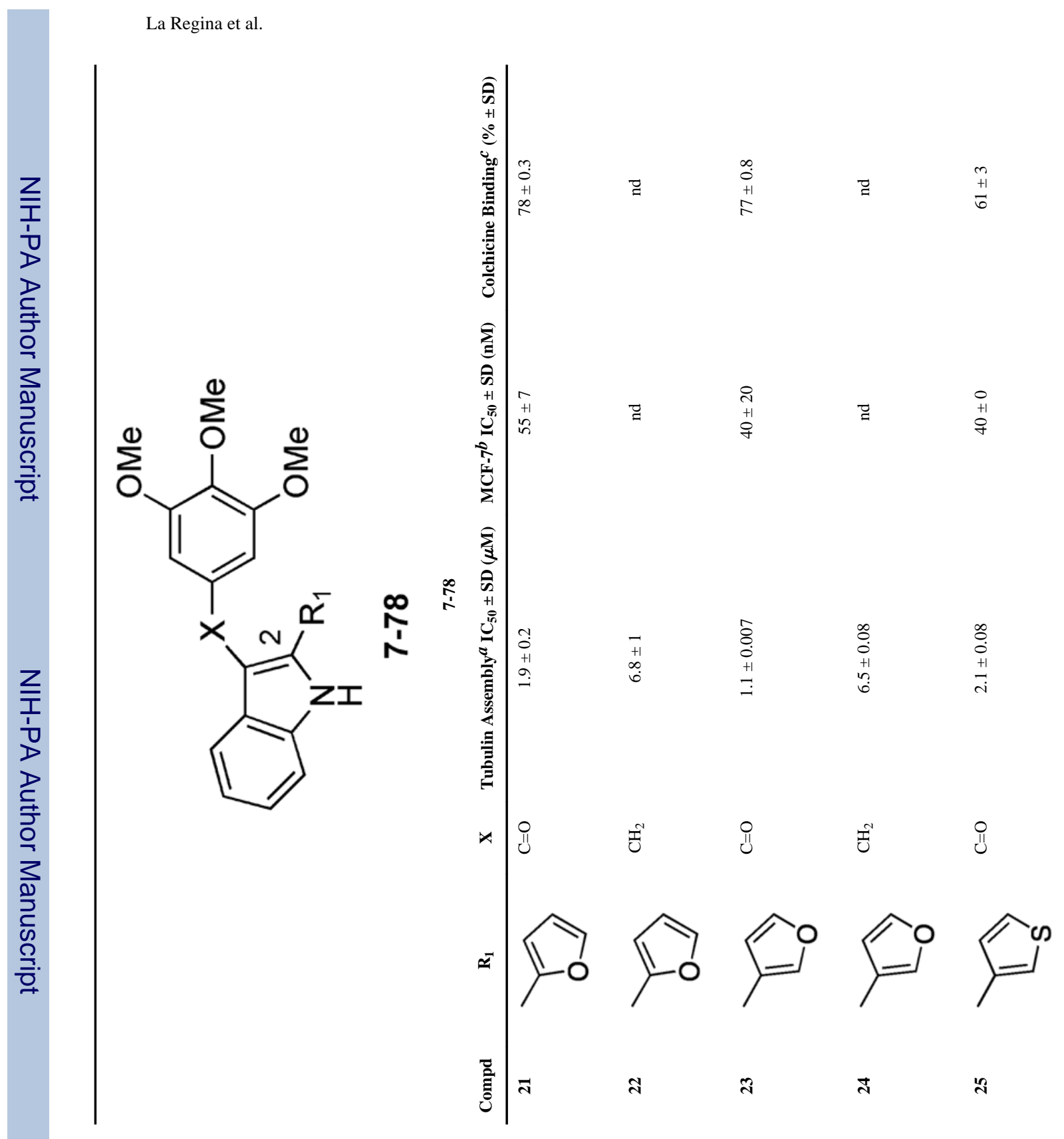

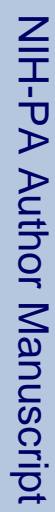

JMed Chem. Author manuscript; available in PMC 2014 January 10. 


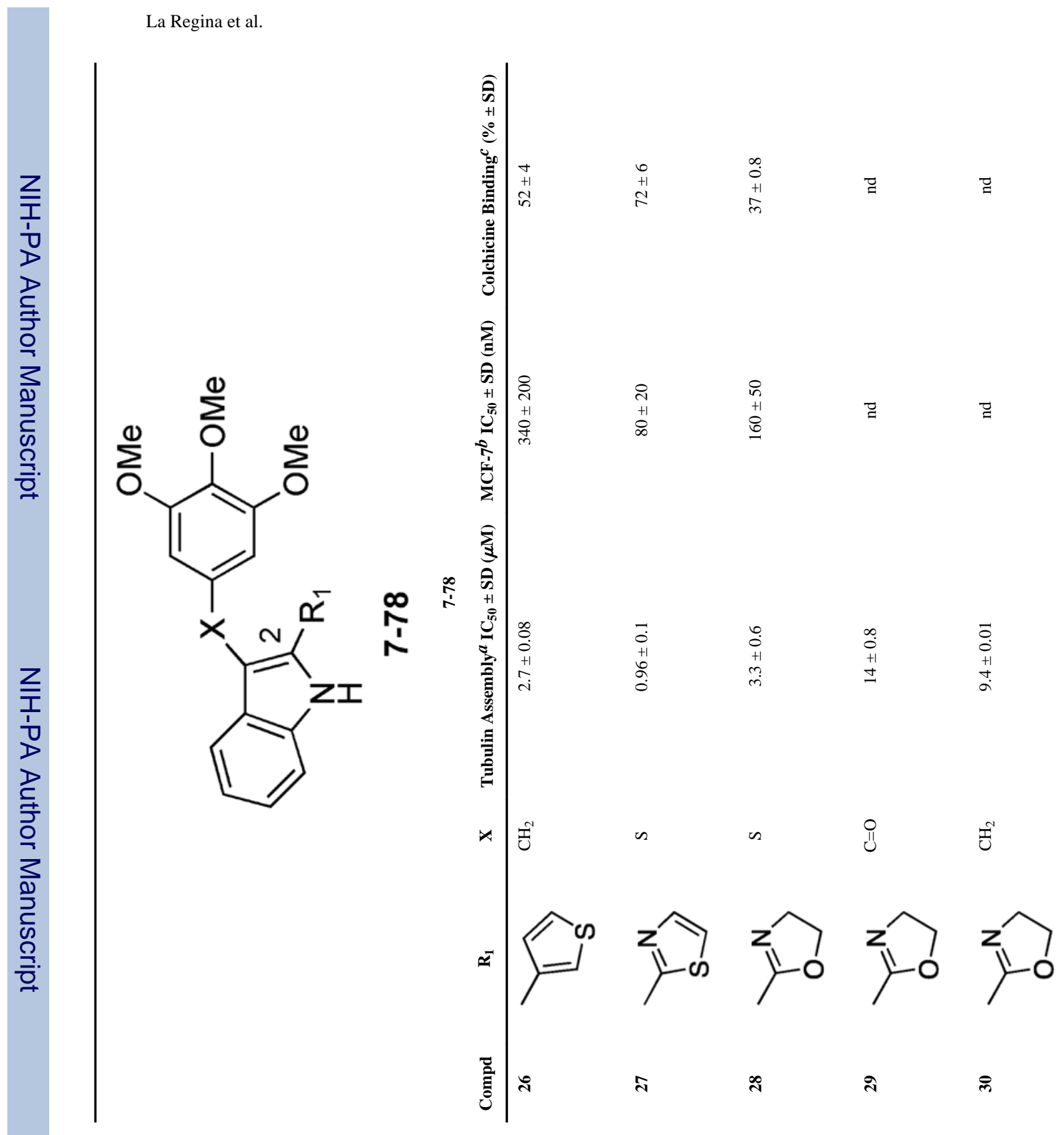




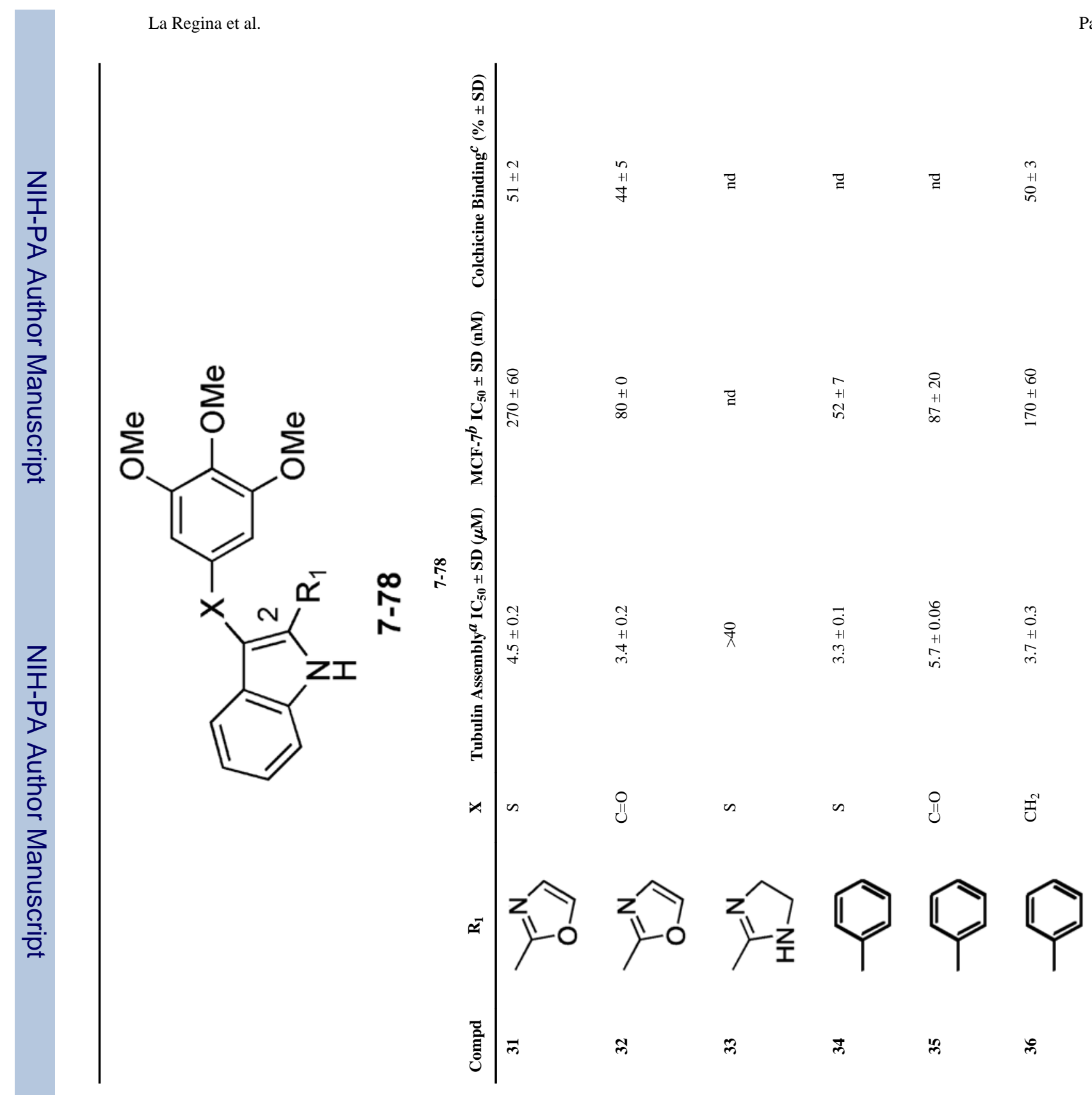

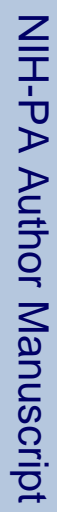

JMed Chem. Author manuscript; available in PMC 2014 January 10. 


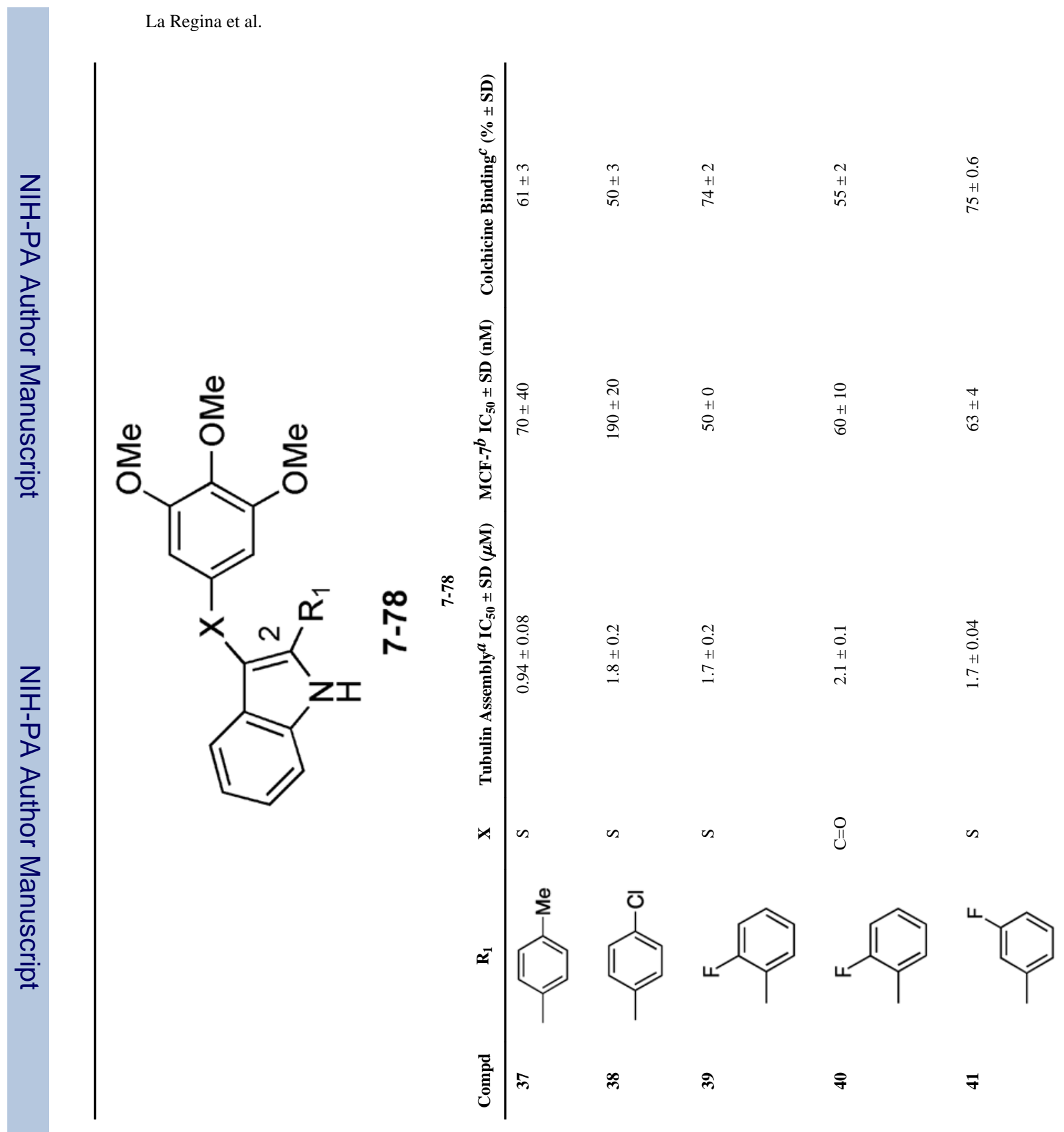

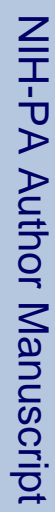

JMed Chem. Author manuscript; available in PMC 2014 January 10. 


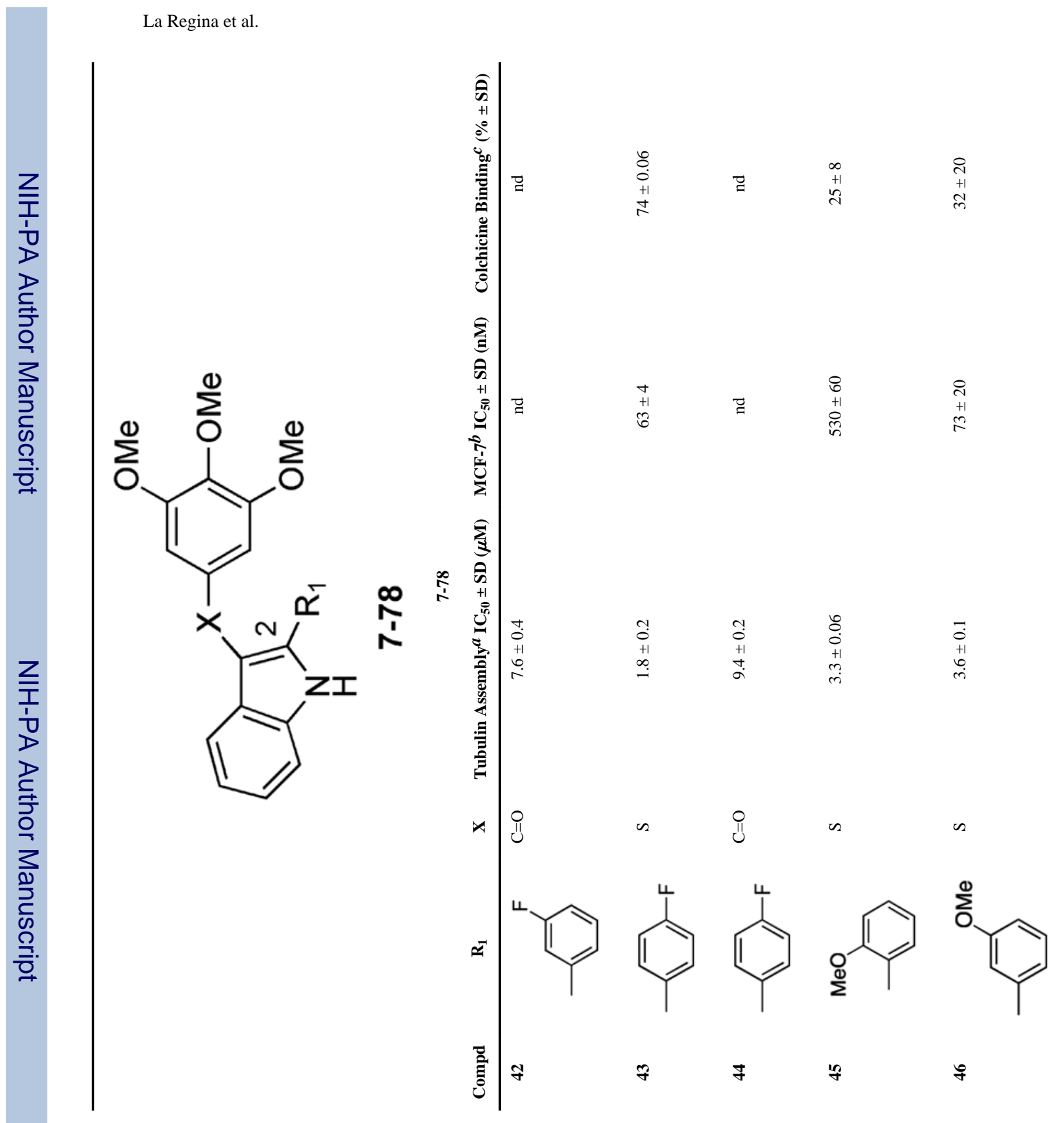

JMed Chem. Author manuscript; available in PMC 2014 January 10. 


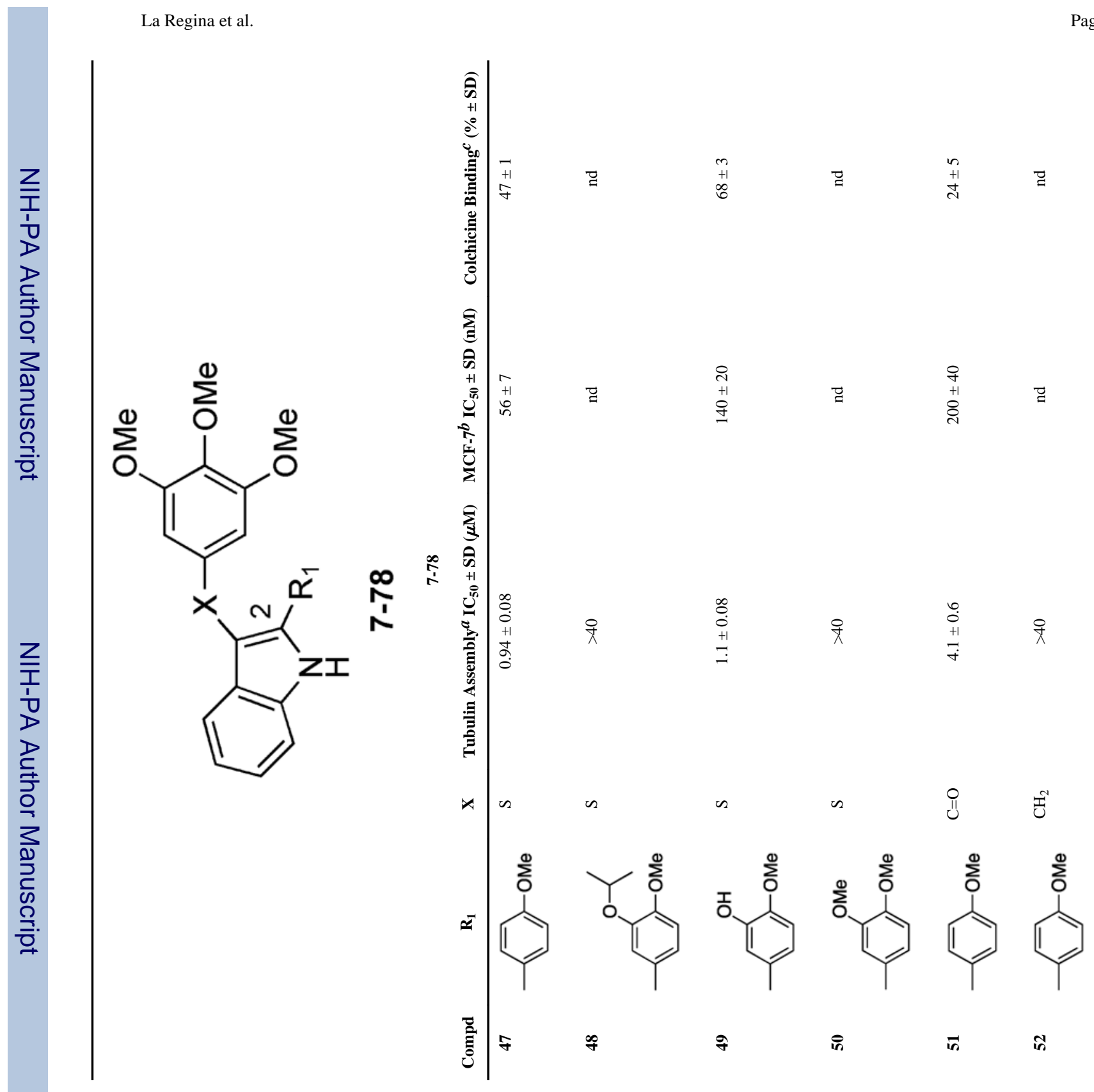

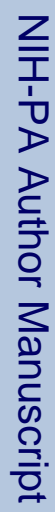

JMed Chem. Author manuscript; available in PMC 2014 January 10. 


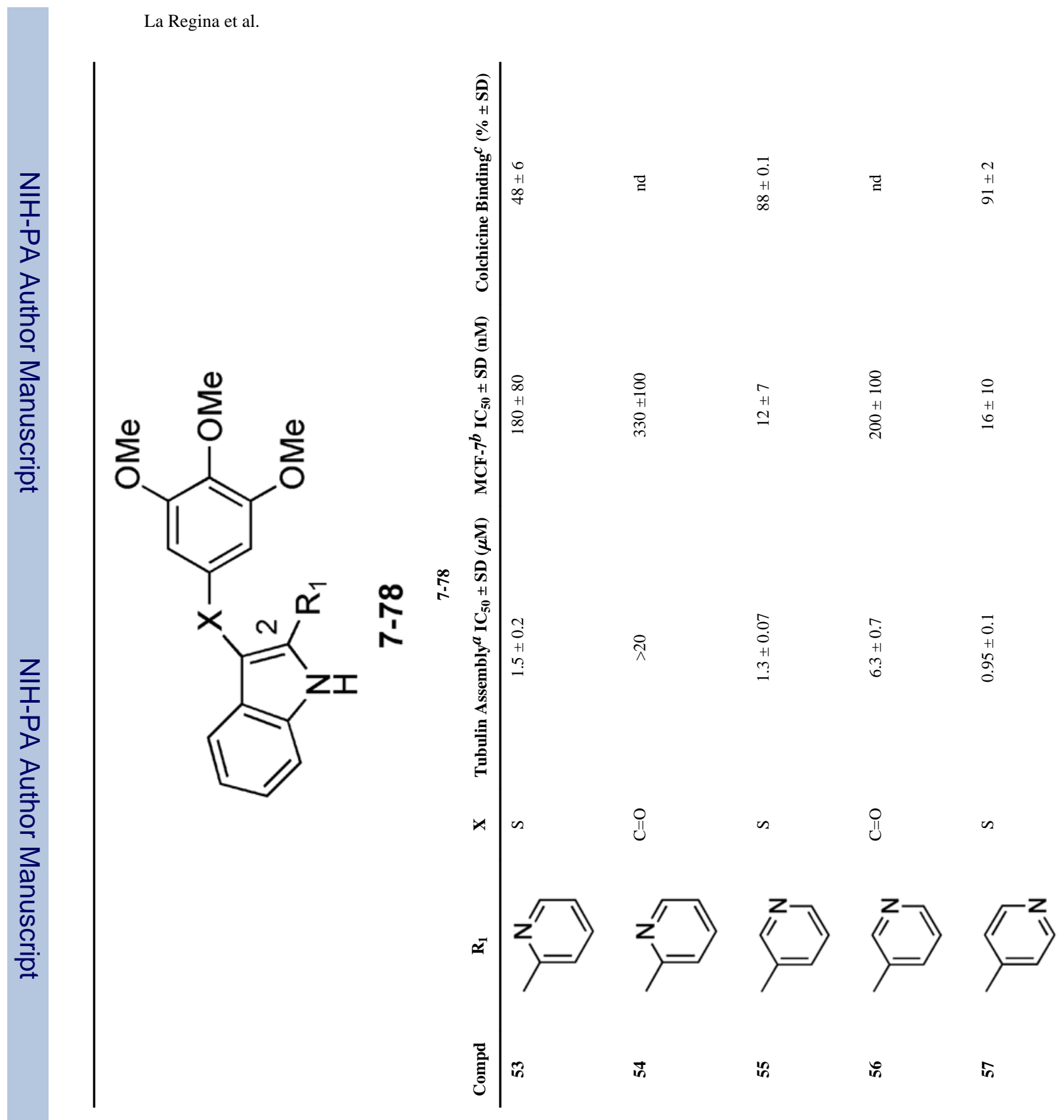

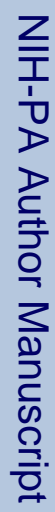

JMed Chem. Author manuscript; available in PMC 2014 January 10. 


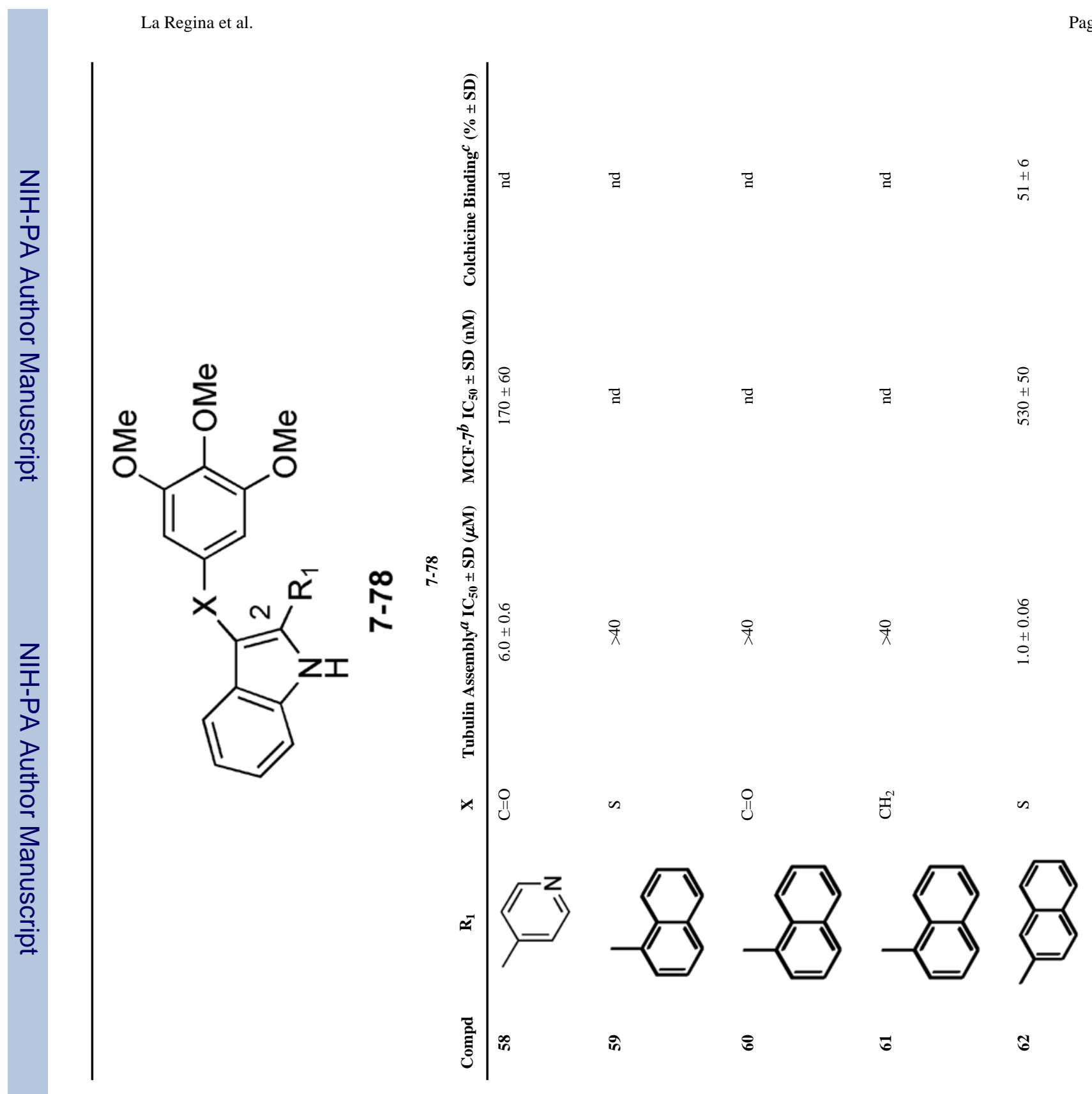

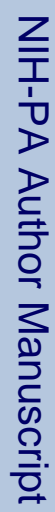

JMed Chem. Author manuscript; available in PMC 2014 January 10. 


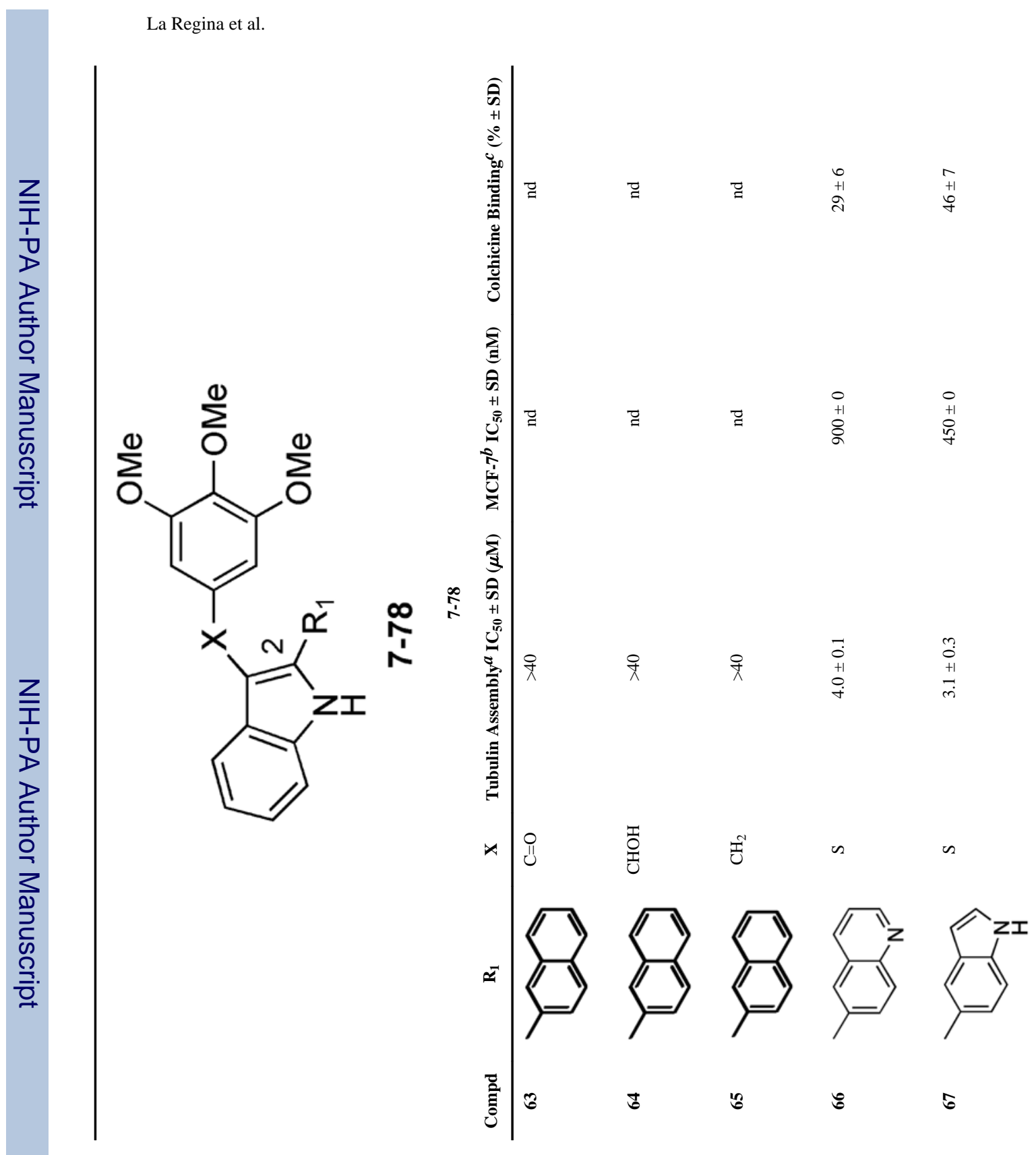

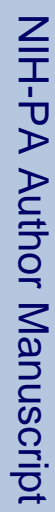

JMed Chem. Author manuscript; available in PMC 2014 January 10. 


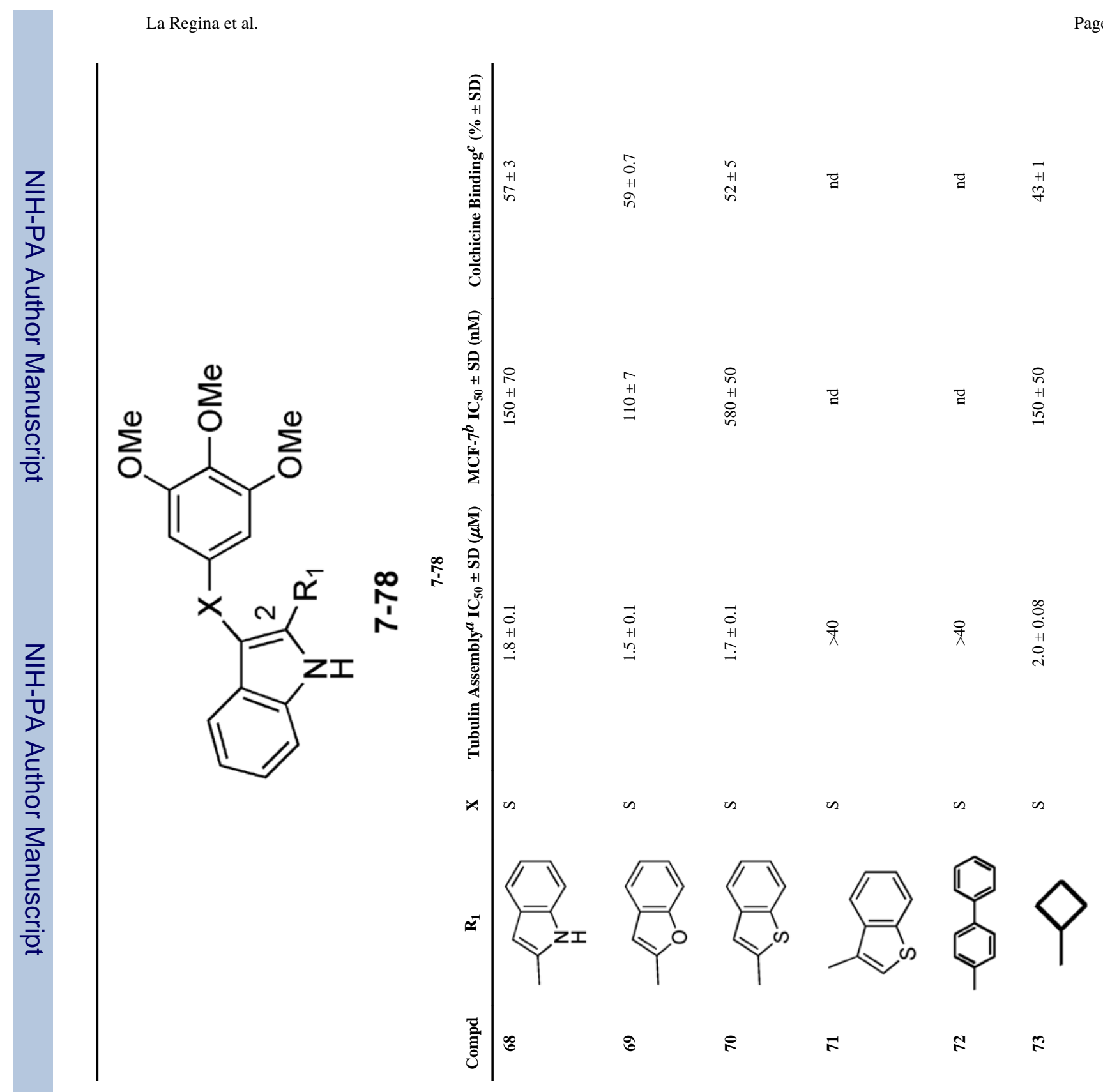

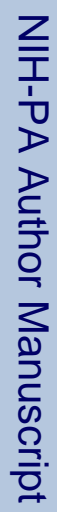

JMed Chem. Author manuscript; available in PMC 2014 January 10. 


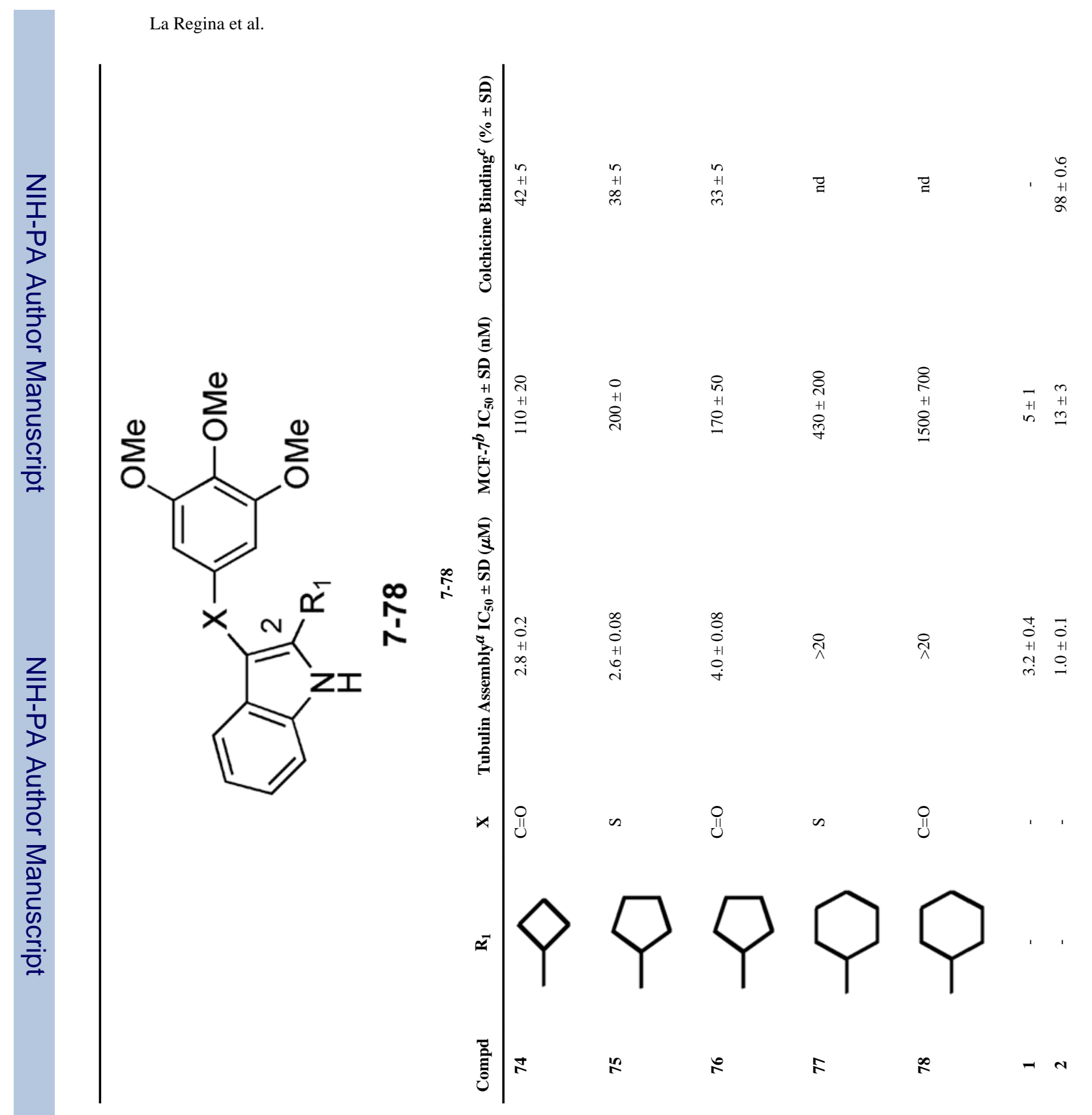

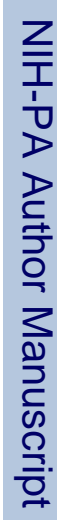

JMed Chem. Author manuscript; available in PMC 2014 January 10. 


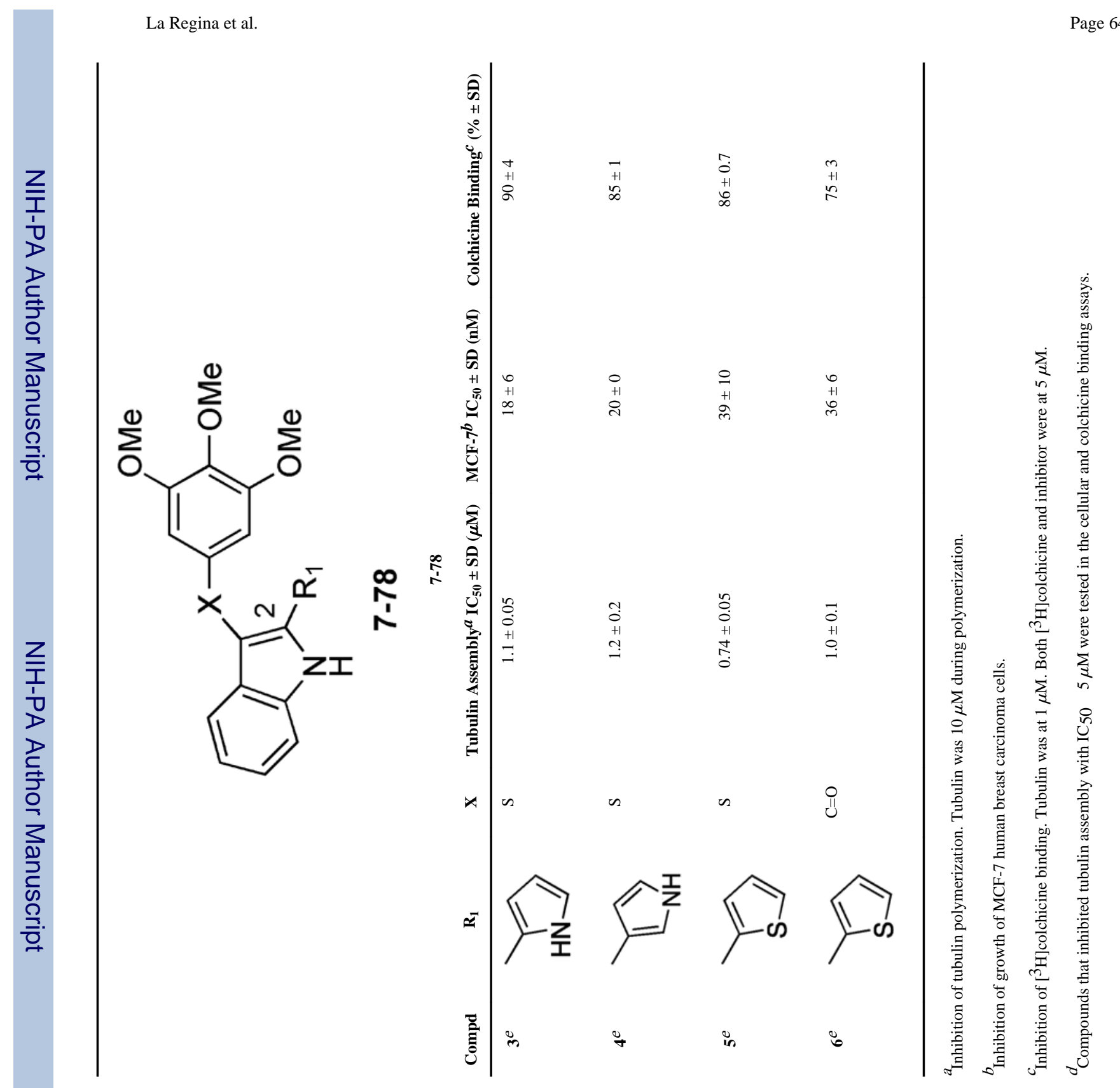

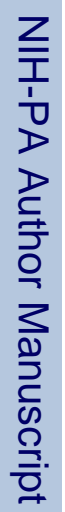

JMed Chem. Author manuscript; available in PMC 2014 January 10. 


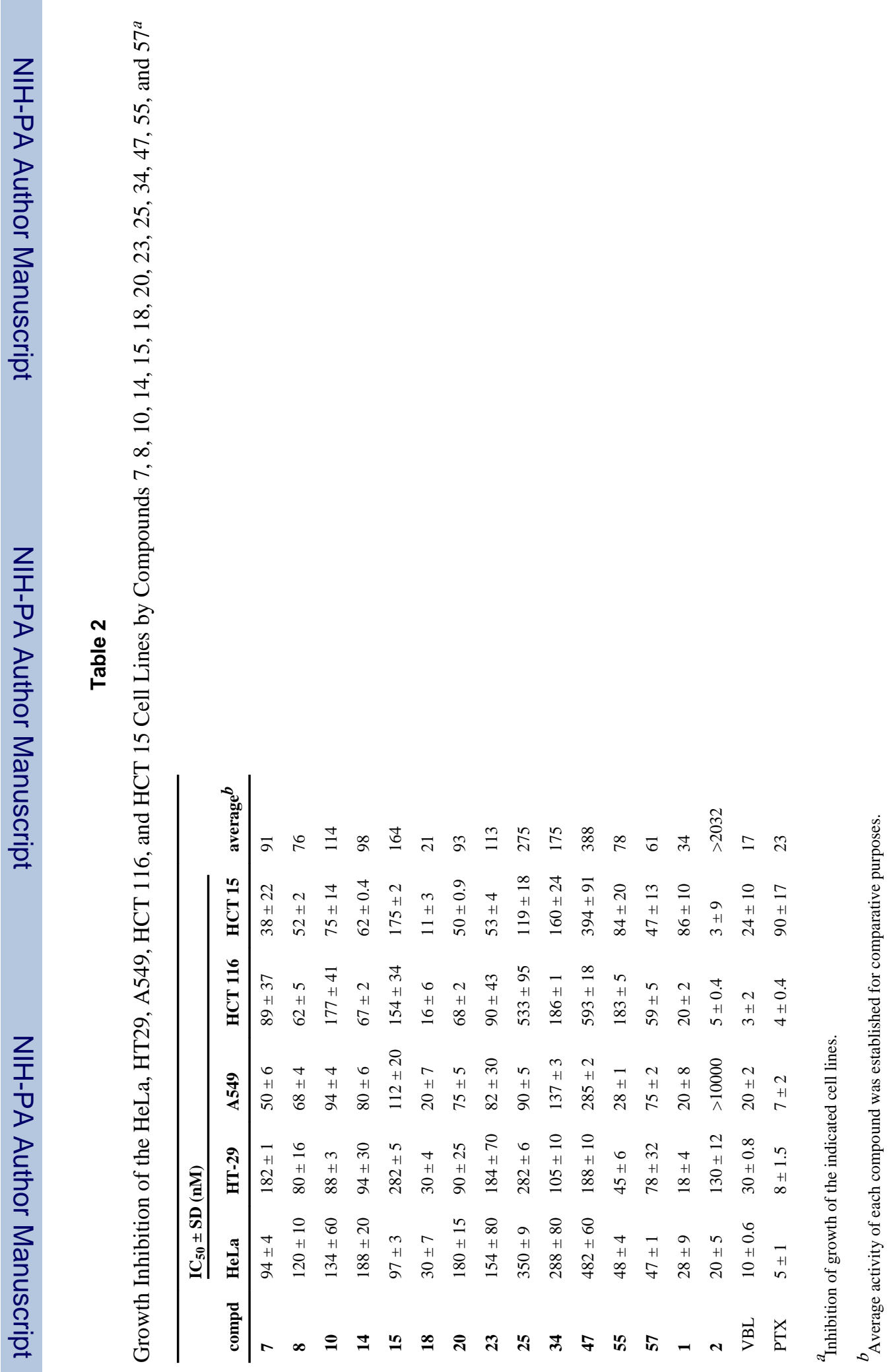


Table 3

Growth Inhibition of the OVCAR-8 and NCI/ADR-RES and Messa and Messa/Dx Cell Lines by Compounds 18, 20, 55, and 57 and Reference Compounds VRB, VBL, PTX, 1, and $2^{a}$

\begin{tabular}{|c|c|c|c|c|}
\hline \multirow[b]{2}{*}{ compd } & \multicolumn{3}{|c|}{$\mathrm{IC}_{50} \pm \mathrm{SD}(\mathrm{nM})$} & \multirow[b]{2}{*}{ Messa/Dx5 $5^{b}$} \\
\hline & OVCAR-8 & NCI/ADR-RES & Messa $^{b}$ & \\
\hline 18 & $16 \pm 6$ & $20 \pm 10$ & $7 \pm 0.3$ & $13 \pm 3$ \\
\hline 20 & $28 \pm 10$ & $15 \pm 7$ & $45 \pm 4$ & $39 \pm 6$ \\
\hline 55 & $17 \pm 6$ & $23 \pm 10$ & $60 \pm 7$ & $71 \pm 2$ \\
\hline 57 & $7.0 \pm 3$ & $14 \pm 7$ & $49 \pm 2$ & $53 \pm 14$ \\
\hline 1 & $\mathrm{nd}^{c}$ & $\mathrm{nd}^{c}$ & $11 \pm 6$ & $329 \pm 166$ \\
\hline 2 & $1.3 \pm 0.6$ & $1.3 \pm 0.6$ & $2.7 \pm 2$ & $2.6 \pm 1$ \\
\hline VRB & $300 \pm 0$ & $5000 \pm 1000$ & $\mathrm{nd}^{c}$ & $\mathrm{nd}^{c}$ \\
\hline VBL & $15 \pm 7$ & $200 \pm 0$ & $3 \pm 2$ & $144 \pm 61$ \\
\hline PTX & $5.0 \pm 2$ & $3300 \pm 1000$ & $4 \pm 1$ & $1764 \pm 477$ \\
\hline
\end{tabular}


Table 4

Metabolic Stability with Human and Mouse Liver Microsomes ${ }^{a}$ and Aqueous Solubility of Compounds 18, 20, 55 , and 57

\begin{tabular}{|c|c|c|c|}
\hline \multirow[b]{2}{*}{ compd } & \multicolumn{2}{|l|}{ \% remaining at $30 \mathrm{~min}^{b}$} & \multirow[b]{2}{*}{ solubility $(\mu \mathrm{M})^{b, d}$} \\
\hline & human liver microsomes & mouse liver microsomes & \\
\hline 18 & $48.6 \pm 1.9$ & $10.3 \pm 0.4$ & $64.5 \pm 0.7$ \\
\hline 20 & $12.0 \pm 1.1$ & $0.6 \pm 0.1$ & $4.0 \pm 0.1$ \\
\hline 55 & $19.3 \pm 0.9$ & $2.8 \pm 0.3$ & $20.5 \pm 0.7$ \\
\hline 57 & $17.7 \pm 0.5$ & $7.9 \pm 0.2$ & $5.0 \pm 0.1$ \\
\hline 7-ethoxycoumarin ${ }^{c}$ & $6.6 \pm 0.2$ & $0.07 \pm 0.02$ & \\
\hline propranolol $^{c}$ & $54.1 \pm 0.4$ & $20.6 \pm 0.5$ & \\
\hline \multicolumn{4}{|c|}{ a Metabolic stability: $>50$, good; $10-50$, medium; $<10$, low. } \\
\hline \multicolumn{4}{|c|}{ 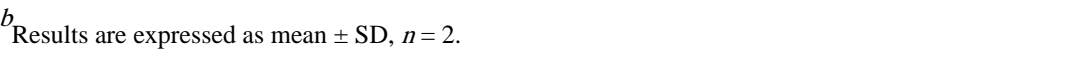 } \\
\hline \multicolumn{4}{|c|}{$\begin{array}{l}c_{\text {The standard compounds } 7 \text {-ethoxycoumarin and propranolol showed metabolic stability in agreement with the literature and internal validation }} \\
\text { data. } 36\end{array}$} \\
\hline
\end{tabular}


